University of Tennessee Health Science Center

UTHSC Digital Commons

\title{
Impact of Adjuvants on the Antibody Responses to Pre-pandemic H5N1 Influenza Vaccines
}

\author{
Ali Hassan Ellebedy \\ University of Tennessee Health Science Center
}

Follow this and additional works at: https://dc.uthsc.edu/dissertations

Part of the Medical Immunology Commons, and the Virus Diseases Commons

\section{Recommended Citation}

Ellebedy, Ali Hassan, "Impact of Adjuvants on the Antibody Responses to Pre-pandemic H5N1 Influenza Vaccines" (2011). Theses and Dissertations (ETD). Paper 75. http://dx.doi.org/10.21007/ etd.cghs.2011.0082.

This Dissertation is brought to you for free and open access by the College of Graduate Health Sciences at UTHSC Digital Commons. It has been accepted for inclusion in Theses and Dissertations (ETD) by an authorized administrator of UTHSC Digital Commons. For more information, please contact jwelch30@uthsc.edu. 


\title{
Impact of Adjuvants on the Antibody Responses to Pre-pandemic H5N1 Influenza Vaccines
}

\author{
Abstract \\ Human influenza pandemics occur when influenza viruses to which the population has little or no \\ immunity emerge and acquire the ability to transmit among humans. Since their emergence in 1996, \\ human infections with highly pathogenic avian influenza A (H5N1) viruses presented a serious public \\ health challenge. Additionally, H5N1 viruses caused significant agricultural and economic losses in the \\ communities it has affected. Human infections with these viruses are rare but when they occur, these \\ infections are highly fatal. A greater public health concern stems from the rapid evolution displayed by \\ these viruses so far, which in turn might result in viruses able to cause sustained and widespread \\ human-to-human transmission.
}

The development of H5N1 vaccines that can induce protective antibody responses is the cornerstone of the global efforts to address this pandemic threat. However, it was repeatedly shown in clinical trials that (at comparable antigen doses) candidate human H5N1 influenza vaccines generally elicit lower immune responses than seasonal human influenza vaccines. In addition, the evolution of H5N1 viruses into at least 10 antigenically distinct clades and multiple subclades suggests that an optimal H5N1 vaccine should confer cross-reactivity against H5N1 viruses from other clades. Therefore, the WHO has recommended the use of adjuvants especially the oil-in-water emulsions (such as MF59) in combination with $\mathrm{H} 5 \mathrm{~N} 1$ split or subunit vaccines. While the ability of these adjuvants to generally boost the vaccinespecific antibody titers has been well documented, the question of how adjuvants modulate the quality of such responses remains largely unanswered.

First, we studied the impact of two adjuvants that are licensed with human influenza vaccines, MF59 and alum on the kinetics of developing protective antibody responses to subunit H5N1 vaccines in the ferret model. With a single immunization regimen, we found that including adjuvants in the vaccine formulation was essential for protection against a lethal H5N1 virus challenge. Adjuvanted vaccines provided protection against lethality when administered as early as 7 days prior to challenge and protection against challenge-associated morbidity when administered 14 days or longer prior to challenge.

We also examined the breadth of the antibody responses to adjuvanted vs. unadjuvanted H5N1 vaccines. Previous studies have suggested that the oil-in-water emulsion adjuvanted vaccines did not only elicit higher antibody titers against homologous H5N1 strains, but also against representative isolates of different clades. Our data clearly showed that indeed MF59-adjuvanted H5N1 vaccines elicited a quantitatively greater $\mathrm{H} 5$-specific antibody response than the alum-adjuvanted or the unadjuvanted vaccine. However, for the most part, the specificity of these antibodies as determined by binding to $\mathrm{H} 5$ antigen microarray and competitive ELISA assays was not different than induced by alum or vaccine alone.

Finally, we tested the contribution of the cytosolic innate immune sensing complex known as the NLRP3 (also known as cryopyrin, CIAS1, or Nalp3) inflammasome is in the adjuvant effect of MF59. It was recently shown that activation of the NLRP3 inflammasome is essential for the adjuvant effect of alum. Our data clearly demonstrated that while the NLRP3 is dispensable for the antibody responses to MF59-adjuvanted H5N1 vaccines, ablation of the adapter molecule ASC abrogated these responses.

\section{Document Type}

Dissertation 


\section{Degree Name}

Doctor of Philosophy (PhD)

\section{Program}

Biomedical Sciences

\section{Research Advisor}

Richard J. Webby, Ph.D.

\section{Keywords}

Adjuvants, H5N1, Influenza, MF59, Vaccines

\section{Subject Categories}

Diseases | Medical Immunology | Medical Sciences | Medicine and Health Sciences | Virus Diseases 
IMPACT OF ADJUVANTS ON THE ANTIBODY RESPONSES TO PRE-PANDEMIC H5N1 INFLUENZA VACCINES

\author{
A Dissertation \\ Presented for \\ The Graduate Studies Council \\ The University of Tennessee \\ Health Science Center
}

\author{
In Partial Fulfillment \\ Of the Requirements for the Degree \\ Doctor of Philosophy \\ From The University of Tennessee
}

By

Ali Hassan Ellebedy

May 2011 
Portions of Chapter 1 (C) 2009 and 2011 by Elsevier B.V. Chapter 4 (C) 2011 by the National Academy of Sciences. All other material (C) 2011 by Ali Hassan Ellebedy. All rights reserved. 


\section{DEDICATION}

“All that I am, or hope to be, I owe to my angel mother" Abraham Lincoln. 


\section{ACKNOWLEDGEMENTS}

I would like to deeply thank my mentor, Dr. Richard Webby, for giving me the opportunity to work in his lab and for the excellent training I have received.

I would also like to thank my committee members, Dr. Tony Marion, Dr. Julia Hurwitz, Dr. Michael Whitt, and Dr. Mark Sangster for their guidance and support during my studies.

Special thanks to Dr. Robert G. Webster, Dr. Paul Thomas, Dr. Jacco Boon, Dr. Scott Brown, Dr. Thirumala-Devi Kanneganti and Dr. Stephanie Sonnenberg for their continuous advice, support and friendship.

Thanks also to all of the members of Webby's lab and of the Flu Group at St. Jude Children's Research Hospital.

This work was funded by the National Institute of Allergy and Infectious Diseases, the National Institutes of Health, under contract number HHSN266200700005C, and the American Lebanese Syrian Associated Charities (ALSAC). 


\begin{abstract}
Human influenza pandemics occur when influenza viruses to which the population has little or no immunity emerge and acquire the ability to transmit among humans. Since their emergence in 1996, human infections with highly pathogenic avian influenza A (H5N1) viruses presented a serious public health challenge. Additionally, $\mathrm{H} 5 \mathrm{~N} 1$ viruses caused significant agricultural and economic losses in the communities it has affected. Human infections with these viruses are rare but when they occur, these infections are highly fatal. A greater public health concern stems from the rapid evolution displayed by these viruses so far, which in turn might result in viruses able to cause sustained and widespread human-to-human transmission.

The development of $\mathrm{H} 5 \mathrm{~N} 1$ vaccines that can induce protective antibody responses is the cornerstone of the global efforts to address this pandemic threat. However, it was repeatedly shown in clinical trials that (at comparable antigen doses) candidate human H5N1 influenza vaccines generally elicit lower immune responses than seasonal human influenza vaccines. In addition, the evolution of $\mathrm{H} 5 \mathrm{~N} 1$ viruses into at least 10 antigenically distinct clades and multiple subclades suggests that an optimal H5N1 vaccine should confer cross-reactivity against $\mathrm{H} 5 \mathrm{~N} 1$ viruses from other clades. Therefore, the WHO has recommended the use of adjuvants especially the oil-in-water emulsions (such as MF59) in combination with H5N1 split or subunit vaccines. While the ability of these adjuvants to generally boost the vaccine-specific antibody titers has been well documented, the question of how adjuvants modulate the quality of such responses remains largely unanswered.
\end{abstract}

First, we studied the impact of two adjuvants that are licensed with human influenza vaccines, MF59 and alum on the kinetics of developing protective antibody responses to subunit $\mathrm{H} 5 \mathrm{~N} 1$ vaccines in the ferret model. With a single immunization regimen, we found that including adjuvants in the vaccine formulation was essential for protection against a lethal $\mathrm{H} 5 \mathrm{~N} 1$ virus challenge. Adjuvanted vaccines provided protection against lethality when administered as early as 7 days prior to challenge and protection against challenge-associated morbidity when administered 14 days or longer prior to challenge.

We also examined the breadth of the antibody responses to adjuvanted vs. unadjuvanted H5N1 vaccines. Previous studies have suggested that the oil-in-water emulsion adjuvanted vaccines did not only elicit higher antibody titers against homologous H5N1 strains, but also against representative isolates of different clades. Our data clearly showed that indeed MF59-adjuvanted H5N1 vaccines elicited a quantitatively greater $\mathrm{H} 5$-specific antibody response than the alum-adjuvanted or the unadjuvanted vaccine. However, for the most part, the specificity of these antibodies as determined by binding to $\mathrm{H} 5$ antigen microarray and competitive ELISA assays was not different than induced by alum or vaccine alone. 
Finally, we tested the contribution of the cytosolic innate immune sensing complex known as the NLRP3 (also known as cryopyrin, CIAS1, or Nalp3)

inflammasome is in the adjuvant effect of MF59. It was recently shown that activation of the NLRP3 inflammasome is essential for the adjuvant effect of alum. Our data clearly demonstrated that while the NLRP3 is dispensable for the antibody responses to MF59-adjuvanted H5N1 vaccines, ablation of the adapter molecule ASC abrogated these responses. 


\section{TABLE OF CONTENTS}

CHAPTER 1. INTRODUCTION ........................................................................................

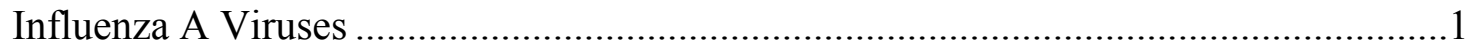

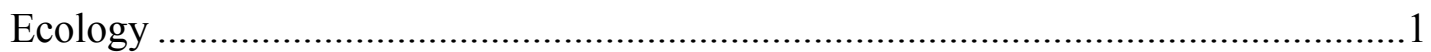

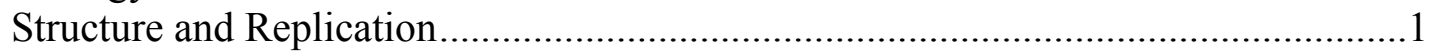

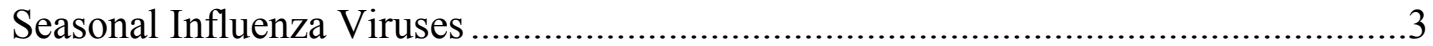

Pandemic Potential of Influenza A Viruses ............................................................

Avian Influenza A Viruses; H5N1 Viruses ...........................................................4

Epidemiology and Genetic Diversity of H5N1 Viruses............................................ 4

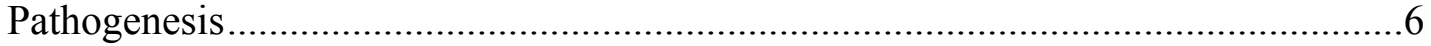

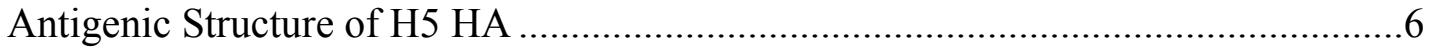

The Immune Response to Influenza Viruses ............................................................

The Innate Immune Responses ..................................................................... 7

The Humoral Immune Responses .................................................................. 9

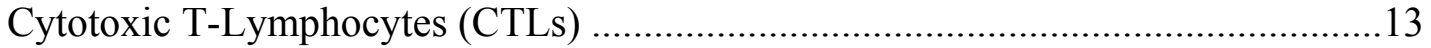

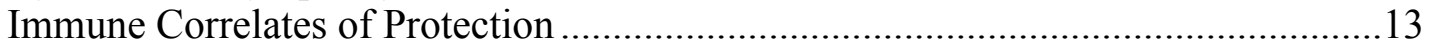

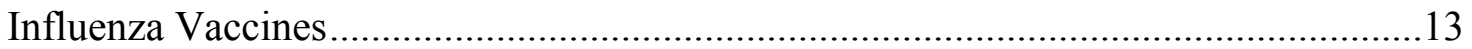

The Challenges of Vaccination against H5N1 .................................................... 13

Inactivated Virus Vaccines ........................................................................ 15

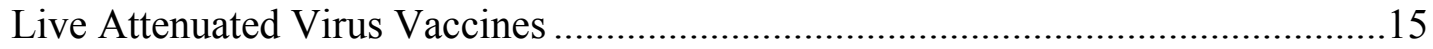

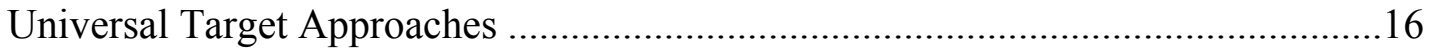

Adjuvants for Vaccines against H5N1 Influenza Viruses .......................................16

Why Are Adjuvants Needed for Influenza Vaccines? ............................................16

General Mechanisms of Action of Adjuvants.................................................. 17

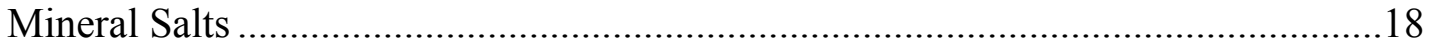

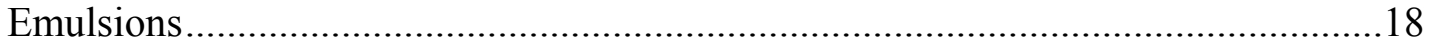

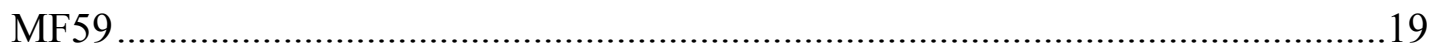

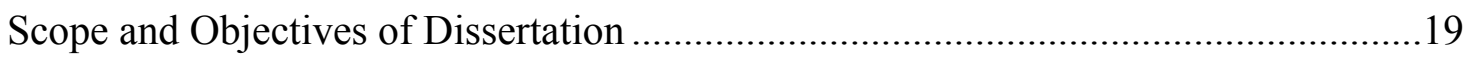

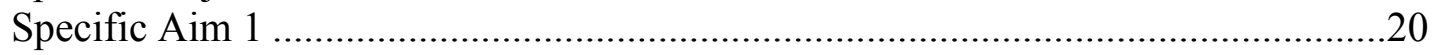

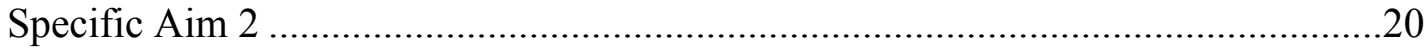

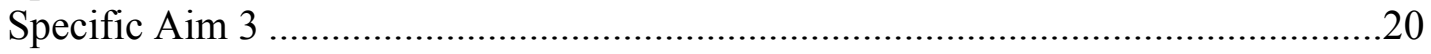

\section{CHAPTER 2. ADJUVANTS ENHANCE THE KINETICS OF PROTECTIVE} ANTIBODY RESPONSES TO H5N1 INFLUENZA VACCINES ...........................21

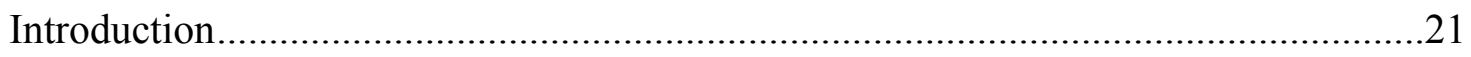

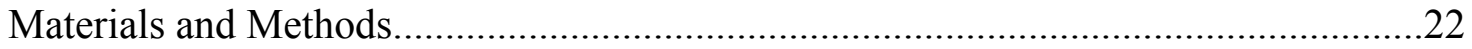

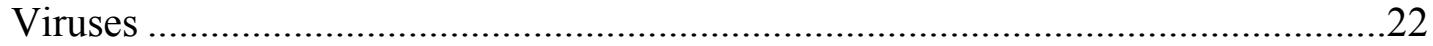

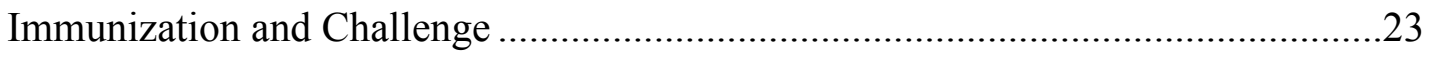

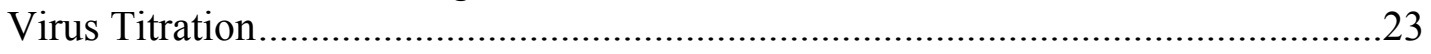

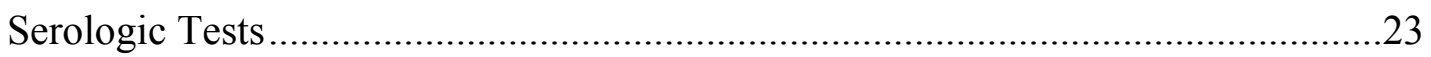

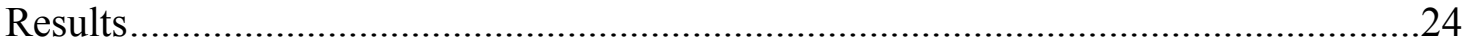

MF59-Adjuvanted H5N1 Vaccine Provides Fast Protection against Challenge-

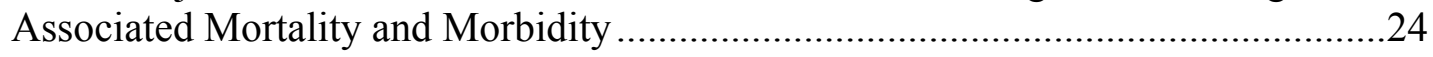

Kinetics of the Antibody Response to the MF59-Adjuvanted H5N1 Vaccines ........24 
Impact of Alum on H5N1 Vaccine-Mediated Protection Kinetics .........................28

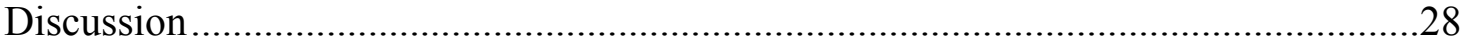

\section{CHAPTER 3. ADJUVANTS' IMPACT ON THE BREADTH OF THE ANTIBODY RESPONSES TO SUBUNIT H5N1 INFLUENZA VACCINES...........34}

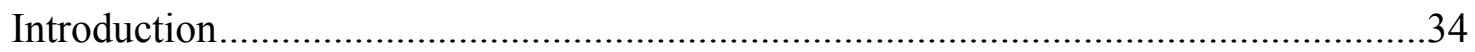

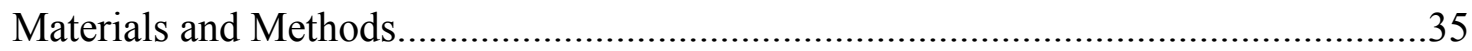

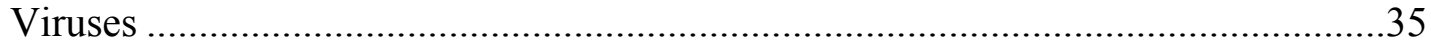

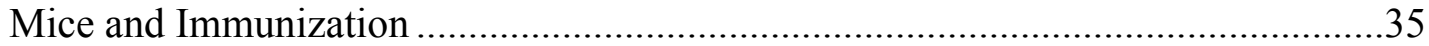

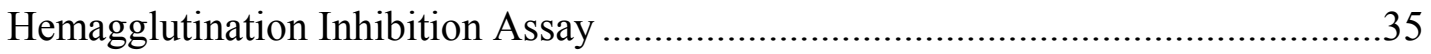

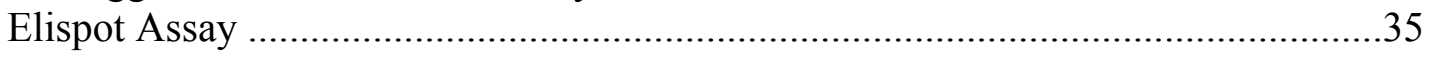

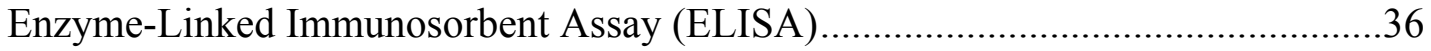

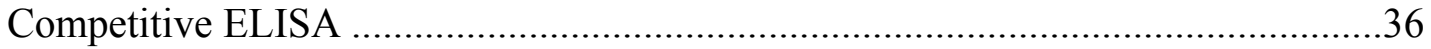

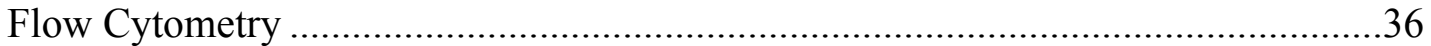

PCR Amplifications and High-Throughput Pyrosequencing and Analysis of the Immunoglobulin Heavy Chain Variable Genes ...................................................... 37

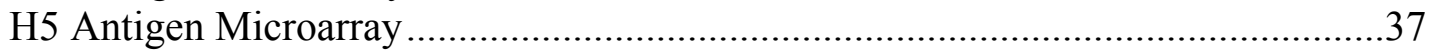

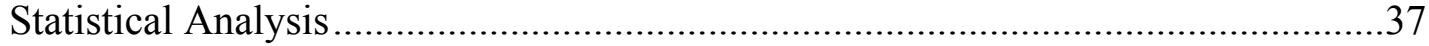

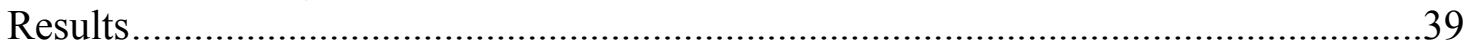

MF59 is Superior to Alum in Priming for Robust H5-Specific Antibody

Responses................................................................................................39

MF59 and Alum Enhance the Affinity Maturation of the B-Cell Response to

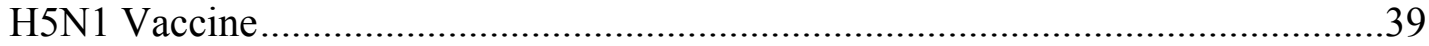

Similar Binding Pattern by Adjuvanted and Unadjuvanted H5 Immune

Serum-Derived IgGs to H5 HA Antigen Microarray..............................................42

Similar Inhibition Pattern by Adjuvanted and Unadjuvanted H5 Immune Sera by

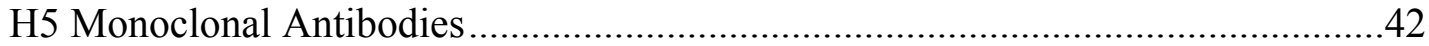

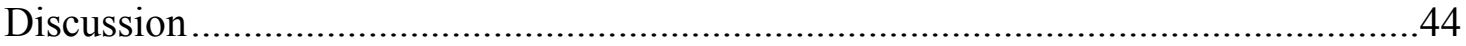

\section{CHAPTER 4. INFLAMMASOME-INDEPENDENT ROLE OF THE APOPTOSIS-ASSOCIATED SPECK-LIKE PROTEIN CONTAINING CARD (ASC) IN THE ADJUVANT EFFECT OF MF59 .............................................47}

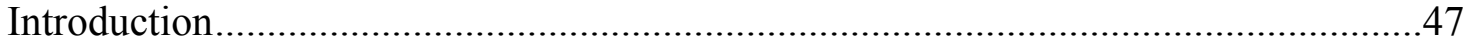

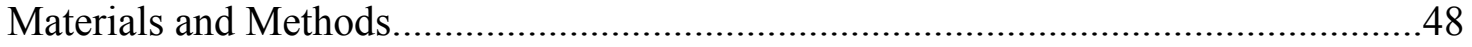

Mice and Immunization ........................................................................... 48

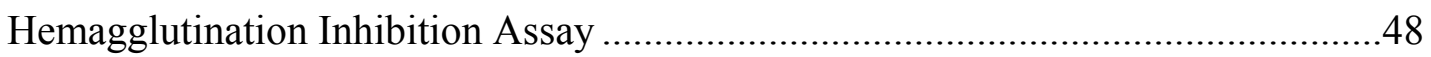

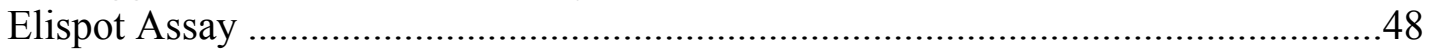

Enzyme-Linked Immunosorbent Assay (ELISA) ................................................49

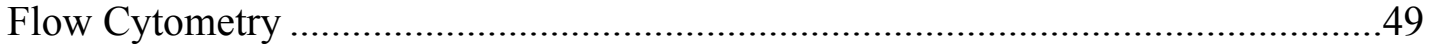

Generation and Analysis of Bone Marrow-Derived Dendritic Cells ........................49

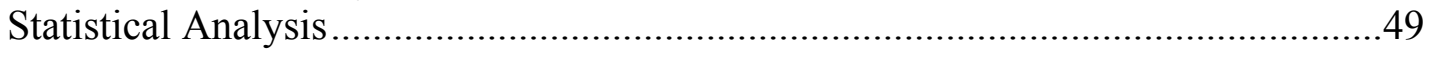

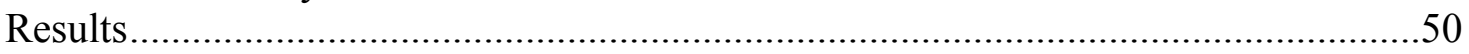

The Humoral Immune Response to MF59-Adjuvanted Vaccines Requires ASC but Not NLRP3 or Caspase-1 ...........................................................................50 ASC Is Required for Germinal Center B-Cell Formation after Immunization with MF59-Adjuvanted Vaccines ...................................................................... 50 
ASC Is Required for Robust Antigen-Specific Recall B-Cell Responses

ASC Expression in Dendritic Cells Is Required for Adequate Production of

Inflammatory Cytokines

Discussion

\section{CHAPTER 5. GENERAL CONCLUSIONS, LIMITATIONS AND FUTURE}

DIRECTIONS

Adjuvants Enhance the Kinetics of Protective Antibody Responses to H5N1

Influenza Vaccines

Implications and Limitations

Future Directions

Adjuvants' Impact on the Breadth of the Antibody Responses to Subunit H5N1

Influenza Vaccines

Implications and Limitations

Future Directions

Inflammasome-Independent Role of the Apoptosis-Associated Speck-Like Protein

Containing CARD (ASC) in the Adjuvant Effect of MF59

Implications and Limitations

Future Directions

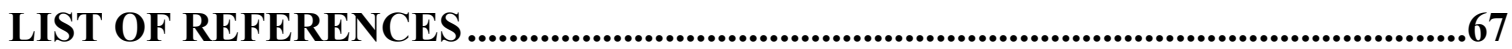

APPENDIX A. IMPACT OF PRIOR SEASONAL INFLUENZA

VACCINATION AND INFECTION ON PANDEMIC A (H1N1) INFLUENZA

VIRUS REPLICATION IN FERRETS.

\section{APPENDIX B. CONTEMPORARY SEASONAL INFLUENZA A (H1N1) \\ VIRUS INFECTION PRIMES FOR A MORE ROBUST RESPONSE TO \\ SPLIT INACTIVATED PANDEMIC INFLUENZA A (H1N1) VIRUS}

VACCINATION IN FERRETS

VITA 


\section{LIST OF TABLES}

Table 1-1. Adjuvants that were shown to significantly boost influenza vaccines in humans.

Table 2-1. Effect of the different vaccination regimen on survival and clinical signs of infection in ferrets challenged with A/Vietnam/1203/04 (H5N1) influenza virus.

Table 2-2. Effect of different vaccination regimen on A/Vietnam/1203/04 (H5N1) influenza virus replication in the upper respiratory tract of ferrets.

Table 2-3. Impact of Alum-adjuvanted H5N1 SU vaccine on survival and clinical signs of infection in ferrets challenged with A/Vietnam/1203/04 (H5N1) influenza virus.

Table 2-4. Serological titers and viral load in ferrets immunized with Alumadjuvanted H5N1 SU vaccine and challenged with A/Vietnam/1203/04 (H5N1) influenza virus. 30

Table 3-1. List of peptide sequences that are used to coat H5 microarray chips .38 


\section{LIST OF FIGURES}

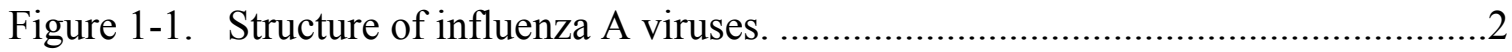

Figure 1-2. Virulence factors and phylogenetic analysis of H5N1 influenza A

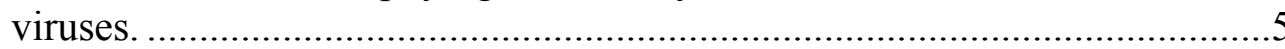

Figure 1-3. The antigenic structure of the H5 HA from A/Vietnam/1203/2004 (H5N1).

Figure 1-4. Innate immune pathways involved in recognition of viruses. 10

Figure 1-5. Mechanisms of antibody mediated immunity against influenza viruses......11

Figure 1-6. The estimated time line for the current influenza vaccine manufacturing process

Figure 2-1. Weight loss in the unadjuvanted and MF59-adjuvanted groups 5 days post challenge with A/Vietnam/1203/04 (H5N1) virus.

Figure 3-1. Superior priming with MF59-adjuvanted H5N1 vaccines in comparison to the alum-adjuvant or the unadjuvanted formulations.

Figure 3-2. Increased affinity maturation of the B-cell responses mediated by alumand MF59-adjuvanted $\mathrm{H} 5 \mathrm{~N} 1$ vaccines.

Figure 3-3. Similar pattern of binding of immune sera to $\mathrm{H} 5$ antigen microarrays.

Figure 3-4. Equivalent inhibition of adjuvanted and unadjuvanted H5 immune sera by $\mathrm{H} 5$ human monoclonal antibodies.

Figure 4-1. Defective humoral immune response to MF59-adjuvanted H5N1 vaccines in ASC knockout mice.

Figure 4-2. Anti-H5 antibody responses in $\mathrm{ASC}^{-/}$are not generally defective.

Figure 4-3. Defective germinal center B-cell development in $\mathrm{ASC}^{-/ 2}$ after immunization with MF59-adjuvanted H5N1 subvirion vaccine.

Figure 4-4. Poor H5-specific antibody recall responses in immunized $\mathrm{ASC}^{-/-}$mice.......56

Figure 4-5. Defective inflammatory responses in $\mathrm{ASC}^{-/}$bone marrow-derived dendritic cells (BMDCs) to MF59. 


\section{LIST OF ABBREVIATIONS}

\begin{tabular}{|c|c|}
\hline AFCs & Antibody Forming Cells \\
\hline AP-1 & Activator Protein 1 \\
\hline APCs & Antigen Presenting Cells \\
\hline $\mathrm{ASC}$ & Apoptosis-Associated Speck-Like Protein Containing a CARD \\
\hline BMDCs & Bone Marrow Derived Dendritic Cells \\
\hline CARD & Caspase Activation and Recruitment Domain \\
\hline CDC & US Centers for Disease Control and Prevention \\
\hline CFA & Complete Freund's Adjuvant \\
\hline CTLs & Cytotoxic T-Lymphocytes \\
\hline DAMPs & Danger Associated Molecular Patterns \\
\hline DCs & Dendritic Cells \\
\hline dsRNA & Double-Stranded RNA \\
\hline ELISA & Enzyme-Linked Immunosorbent Assay \\
\hline $\mathrm{GCs}$ & Germinal Center B-Cells \\
\hline H1N1pdm & 2009 Pandemic Influenza A Virus (H1N1) \\
\hline HA & Hemagglutinin \\
\hline HI & Hemagglutination Inhibition \\
\hline HPAI & Highly Pathogenic Avian Influenza \\
\hline IAVs & Influenza A Viruses \\
\hline IFN & Interferon \\
\hline IL & Interleukin \\
\hline IPAF & ICE-Protease Activating Factor \\
\hline ISRE & Interferon-Sensitive Response Element \\
\hline LAIVs & Live Attenuated Influenza Virus Vaccines \\
\hline LPS & Lipopolysaccharides \\
\hline M1 & Matrix Protein 1 \\
\hline M2 & Matrix Protein 2 \\
\hline M2e & M2 Protein Ectodomain \\
\hline $\mathrm{mAbs}$ & Monoclonal Antibodies \\
\hline MAP & Mitogen-Activated Protein \\
\hline MDA5 & Melanoma Differentiation-Associated Gene 5 \\
\hline MyD88 & Myeloid Differentiation Primary Response Protein 88 \\
\hline NA & Neuraminidase \\
\hline NALP1 & NACHT-LRR-PYD-Containing Protein-1 \\
\hline NEP & Nuclear Export Protein \\
\hline NF-kB & Nuclear Factor Kappa-Light-Chain-Enhancer of Activated B cells \\
\hline NLRP3 & NOD-Like Receptor Family, Pryin Domain Containing 3 \\
\hline NLRs & NOD-Like Receptors \\
\hline NOD & Nucleotide-Binding Oligomerization Domain-Containing Protein \\
\hline NP & Nucleoprotein \\
\hline NS1 & Non Structural Protein 1 \\
\hline NS2 & Non Structural Protein 2 \\
\hline PA & Polymerase Acidic \\
\hline
\end{tabular}


PAMPs

PB1

PB2

PBS

PCs

PRRs

RBCs

RIG-I

RLRs

RNA

RNP

ssRNA

TIV

TLRs

TRIF

WHO
Pathogen Associated Molecular Patterns

Polymerase Basic 1

Polymerase Basic 2

Phosphate-Buffered Saline

Plasma Cells

Pattern Recognition Receptors

Red Blood Cells

Retinoic Acid Inducible Gene I

RIG-Like Receptors

Ribonucleic Acid

Ribonucleoprotein

Single-Stranded RNA

Trivalent Inactivated Influenza Vaccine

Toll-Like Receptors

Toll/IL-1 Domain-Containing Adaptor-Inducing IFN-Beta

World Health Organization 


\title{
CHAPTER 1. INTRODUCTION ${ }^{1}$
}

\author{
Influenza A Viruses
}

\section{Ecology}

Influenza viruses belong to the Orthomyxoviridae family and are antigenically classified into three different classes; A, B and C (1). Aquatic birds are considered the primary reservoir for influenza A viruses (2). Other animal hosts from which influenza A viruses were isolated includes humans, pigs, birds, horses, whales, seals and mink (2). Humans get transiently infected with a limited number of influenza A viruses subtypes. The site of viral replication in aquatic birds is in the cells lining the gastro-intestinal tract (2). In humans, the virus replicates primarily in the respiratory tract. It is not uncommon for influenza A viruses to cross the interspecies barrier which, as it will be discussed below, can lead to serious consequences.

\section{Structure and Replication}

Influenza A viruses are enveloped, negative sense, single stranded RNA viruses with a segmented genome (1). The eight segments of influenza A viral genome code for up to11 proteins. As shown in Fig. 1-1, three of these proteins are expressed on virus surface; first, the hemagglutinin (HA), which is a homotrimeric glycoprotein that is responsible for the initial virus attachment to host epithelial cells and the subsequent virus entry (3). HA also mediates virus fusion with the endosomal membrane, which in turn allows for the virus replication machinery to be transported into the cytosol (3). Second, the neuraminidase (NA), which is a homotetramer that cleaves budding viral particles from the host infected cells (4). Influenza A viruses are further classified based on the antigenicity of their surface HA and NA molecules. To date there are $16 \mathrm{HA}$ subtypes (H1-H16) and 9 NA subtypes (N1-N9) identified (5). The third surface glycoprotein is the matrix protein 2 (M2), produced as a splice variant of the matrix gene and acts as an ion channel ( 6 ) that is responsible for acidifying the viral core, an essential step during viral replication (1). While HA is the major target of virus neutralizing antibodies, NA and M2 are the major targets of the most commonly used anti-influenza antiviral drugs. Matrix protein 1 or M1 binds to the cytosolic domains of HA, NA and M2 forming a septum between the viral membrane and viral internal proteins (1). Four internal viral proteins, the nucleoprotein (NP), the RNA polymerase basic 1 and 2 (PB1 and PB2) and the RNA polymerase acidic (PA), form the ribonucleoprotein (RNP) complex which represents the viral replication apparatus (1).

${ }^{1}$ Parts of this chapter were adapted with permission. A. H. Ellebedy, R. J. Webby, Vaccine 27 Suppl 4, D65 (Nov 5, 2009) (48) and S. A. Valkenburg et al., Microbes Infect 13(5):489-501 (Feb 1, 2011) (45). 


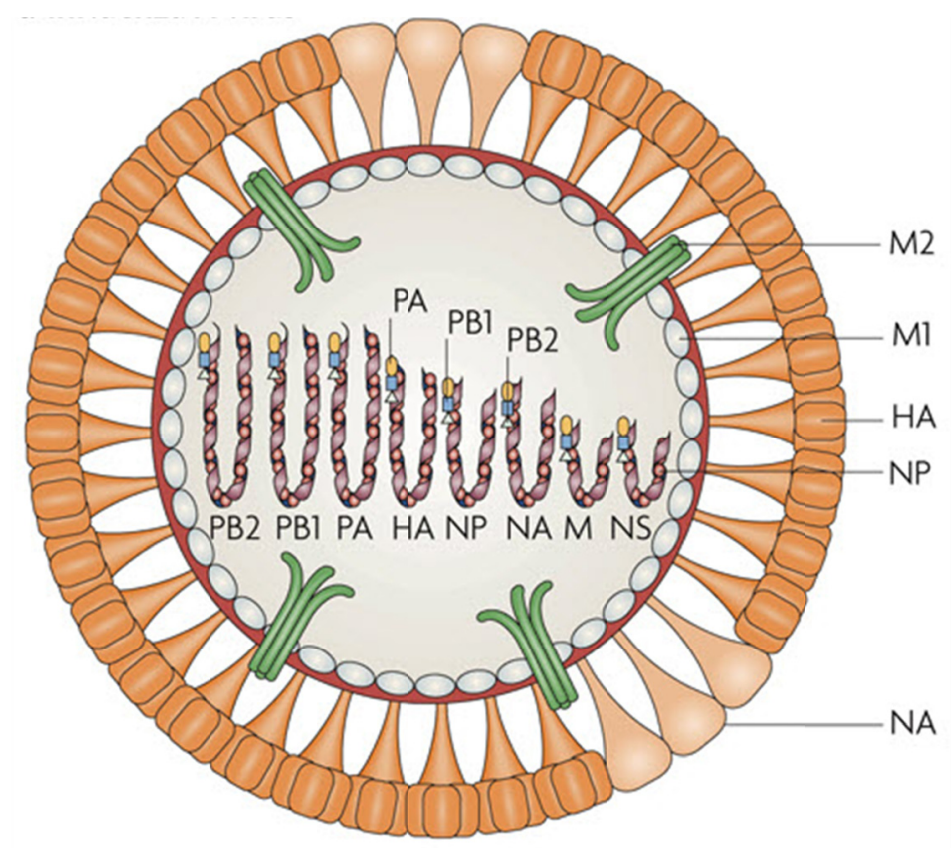

Figure 1-1. Structure of influenza A viruses.

Influenza A virus particles contain eight single-stranded RNA gene segments with two major surface glycoproteins. This schematic was reprinted with permission. K. Subbarao, T. Joseph, Nat Rev Immunol 7, 267 (Apr, 2007) (8).

There is a single non-structural protein or NS1 that is through binding to double stranded RNA, it inhibits host innate antiviral immune response (7). The nuclear export protein or NEP (also known as NS2) is a splice variant of the NS gene and it is essential in exporting newly formed virus RNPs from the infected cell nucleus into its cytosol (1). Finally, PB1-F2, which is produced by an alternate reading frame of the PB1 gene in some strains and recent studies have suggested that it is involved in viral pathogenesis (9).

Influenza A virus replication starts by binding of HA on the viral surface to the sialic acid receptor on the host cell surface. Viral tropism is determined by the type of the terminal sialic acid linkage to galactose on host cells. In general, human influenza viruses bind to sialic acids with $\alpha 2-6$ linkage while avian influenza viruses bind to sialic acid with $\alpha 2-3$ linkage ( $(1)$. Then, the virus enters the host cell by clathrin-coated receptor mediated endocytosis (10). The acidic environment within the endosome causes a conformational change of HA leading to its fusion with the vesicular membrane and the subsequent release of the viral RNP complex into the cytosol (10). The proton influx mediated by the M2 ion channel induces dissociation of the M1 from the viral RNP, resulting in uncoating of the virus. Next, the viral RNPs are translocated to the host cell nucleus where transcription and replication of the viral genome occur. With the help of NEP or NS2, the progeny viral RNPs are translocated into the cytosol and then to the cell membrane where the viral surface protein are assembled. NA cleaves the budding viral 
particle leading to their release from infected cells (10). Finally, for the budding virus particles to be infective, proteolytic cleavage of the HA by host proteases must occur (10). This step is a major determinant of virus tropism.

\section{Seasonal Influenza Viruses}

In the USA, seasonal influenza viruses cause an annual epidemic that usually lasts from the month of November to late April or May with the peak number of infections occurring in February (11). The most affected age populations in terms of infection rate and severity are young children ( $<2$ years old) and the elderly ( $>65$ years old) $(11)$. This is mostly due to the general weakness of the immune system associated with these age groups as well as the lack of preexisting immunity in the young children. Other risk groups include individuals with chronic medical conditions and immunocompromised patients. Annually, seasonal influenza viruses are responsible for approximately 36,000 deaths in the US alone (11).

In the last three decades, three different subtypes of seasonal influenza viruses have circulated in the human population; two influenza A viruses with H1N1 and H3N2 subtypes and influenza B viruses (12). Annual vaccination programs have been developed and are effective in reducing the incidence of seasonal influenza-associated complications (11).

The incubation period after seasonal influenza virus infection ranges from one to four days (11). Typically, it is characterized by mild respiratory symptoms including fever, myalgia, headache, malaise, nonproductive cough, sore throat, and rhinitis (11). The majority of influenza infections resolve within a week (11). However, influenza virus infections can cause primary influenza viral pneumonia; exacerbate underlying medical conditions (e.g., pulmonary or cardiac disease); lead to secondary bacterial pneumonia, sinusitis, or otitis media; or contribute to coinfections with other viral or bacterial pathogens (11).

\section{Pandemic Potential of Influenza A Viruses}

In order for any influenza virus to cause a pandemic, two conditions must be met; 1) the majority of the human population has to be immunologically naïve to that virus and 2) the virus must efficiently transmit between humans. The emergence of such viruses can occur by genetic reassortment of a human influenza virus with a non-human virus leading to a reassortant virus with a novel HA and internal genes that allow it to replicate efficiently in the human host (13). A pandemic influenza virus can also be directly introduced to the human population via interspecies transmission (13). These two mechanisms are not mutually exclusive and a combination of the two processes usually leads to the genesis of a pandemic virus. During the twentieth century, three influenza pandemics occurred (14). The first one was the 1918 Spanish influenza. The causative virus was an avian $\mathrm{H} 1 \mathrm{~N} 1$ virus and the number of people died as a consequence of this 
pandemic is estimated to be 50 million (15). The virus infection was not systemic and secondary bacterial infection was the leading cause of death (15). Recently, the H1N1 virus was recreated using reverse genetics and studies on rodents and non-human primates revealed an aberrantly intense innate immune response associated with virus infection (15). This fact might explain the fact that young adults were the most affected age group.

In 1957, an H2N2 influenza virus caused the second influenza pandemic that is also known as the Asian influenza (10). The virus emerged in Sothern China and the number of deaths within the US is estimated to be 70,000 (16). The virus resulted from a genetic reassortment event with the HA, NA and PB1 genes coming from an avian influenza virus while the rest of genes being contributed by a human virus (16). Unlike the 1918 pandemic, secondary bacterial infection was not the major cause of death.

The last pandemic of the twentieth century was the Hong Kong influenza. The virus emerged in 1968 and has been circulating ever since (16). The pandemic caused 34,000 deaths in the US (16). It is speculated that preexisting immunity against the N2 contributed to the decreased mortality (16).

In April of 2009, the US Centers for Disease Control and Prevention (CDC) reported two cases of a novel H1N1 influenza virus in children in the southern United States (17). It was retrospectively shown that these cases represented the continued spread of this virus, subsequently labeled pandemic A (H1N1) 2009 (H1N1pdm), from an ongoing outbreak in Mexico (18). The virus was quickly sequenced and shown to be a novel reassortant between lineages of influenza viruses known to circulate widely in swine (19). Within a matter of weeks the virus had spread to a number of other continents, signaling the beginning of the first influenza pandemic of the $21^{\text {st }}$ century.

\section{Avian Influenza A Viruses; H5N1 Viruses}

\section{Epidemiology and Genetic Diversity of H5N1 Viruses}

The first identification of a highly pathogenic H5N1 virus was in 1996 and it was from a goose in the Guangdong province of China (20). Since then related viruses have spread on a global scale with confirmed human infections in more than 15 countries (20). As would have been predicted for an influenza virus, H5N1 influenza viruses have evolved into a number of distinct lineages (20). As shown in Fig. 1-2, phylogenic analysis of the HA protein alone has shown that there are at least 10 distinct clades of H5N1 viruses (21). Many of these clades have only been found in avian species in China and the extent to which they circulate is unknown (21). If one considers the clinically significant clades (i.e., those associated with human infections) then this list can be reduced to 6 clades/subclades; 0, 1, 2.1, 2.2, 2.3 and 7 (21). As such, and without any way of predicating which of the clades have any potential for human adaptation, much importance has been placed on increasing the cross reactivity of H5N1 vaccines. 


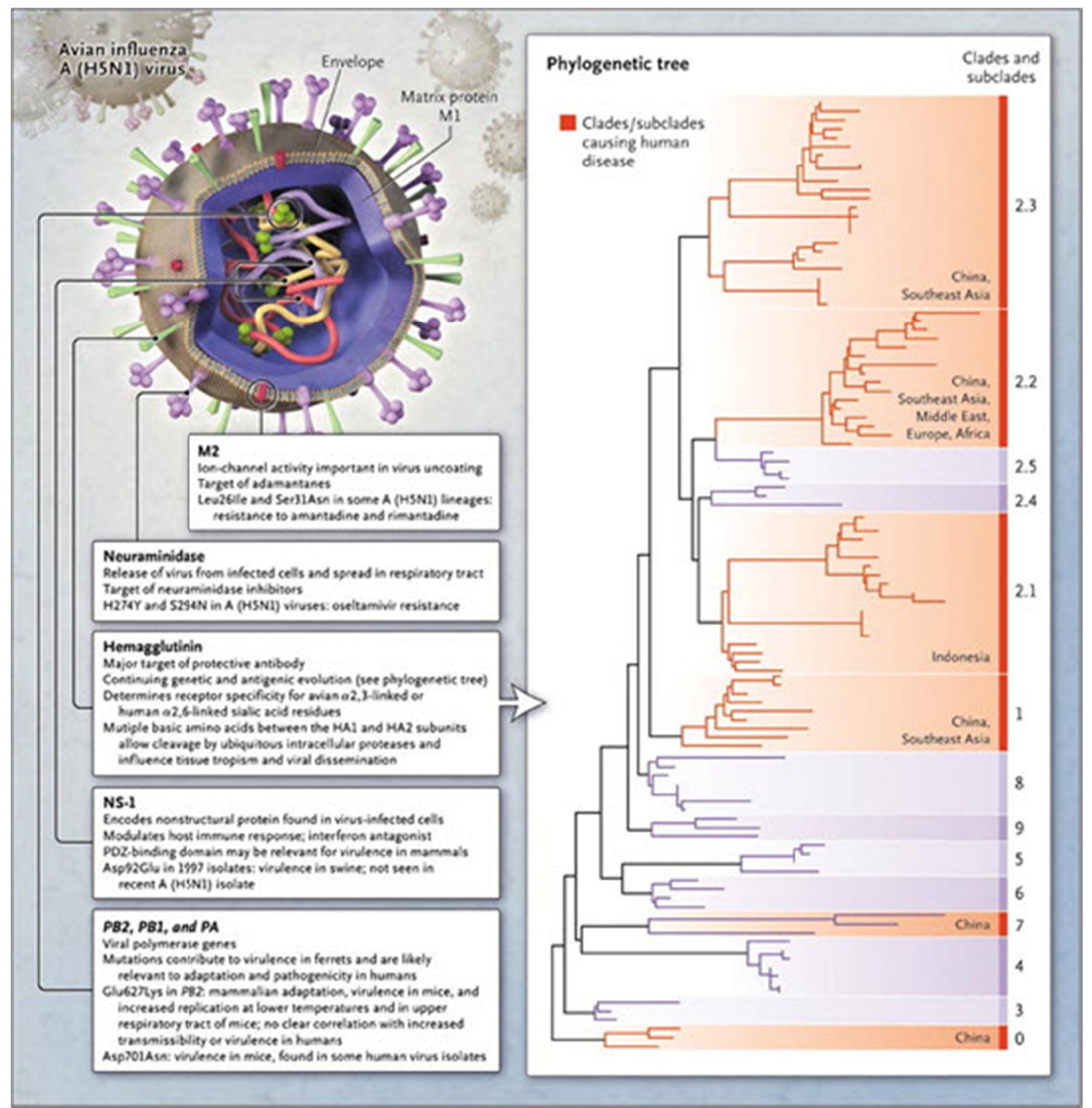

Figure 1-2. Virulence factors and phylogenetic analysis of H5N1 influenza A viruses.

This schematic was reprinted with permission. A. N. Abdel-Ghafar et al., $N$ Engl J Med 358, 261 (Jan 17, 2008) (21). 


\section{Pathogenesis}

Among the different avian influenza A viruses that have crossed the interspecies barrier and infected humans, the highly pathogenic H5N1 viruses were the most severe in terms infection associated morbidity and mortality (22). In Hong Kong in 1997, six out of the eighteen individuals infected with $\mathrm{H} 5 \mathrm{~N} 1$ died (23). There is no clear evidence that human-to-human transmission was established (23). The H5N1 viruses caused the outbreak were believed to be permanently eradicated by culling all the poultry in the Hong Kong markets (23). In 2003, additional human infections caused by H5N1 viruses were reported in Hong Kong (23). To date, the mortality rate among humans infected with avian $\mathrm{H} 5 \mathrm{~N} 1$ viruses is estimated to be $60 \%$ (23). Genetic analyses performed on $\mathrm{H} 5 \mathrm{~N} 1$ viruses isolated from confirmed human cases did not reveal any genetic reassortment between these viruses and human or swine viruses (24). However, coinfections experiments have revealed that the potential of emergence of such reassortants is viable (25). The role of the host genetic component in the susceptibility to infection or the severity of infection associated morbidity was suggested but not confirmed (21).

In general, the incubation period in patients infected with $\mathrm{H} 5 \mathrm{~N} 1$ viruses ranges from two to five days (21). Typically patients present with the regular influenza respiratory symptoms. However, gastrointestinal symptoms including diarrhea have also been reported (21). Pneumonia, acute respiratory distress and multi organ failure are the characteristic symptoms of severe cases and unless intervention with antiviral drugs like oseltamivir starts, those patients likely succumb to infection (21). Unlike the pattern with seasonal influenza viruses, young adults (10-19 years old) are the most affected age population in terms of mortality (26). Autopsies from fatal cases have revealed a massive alveolar damage with evidence of heavy involvement of macrophages and neutrophils. In addition, high levels of proinflammatory cytokines were detected in the serum (26). These findings led to the hypothesis that H5N1 virus associated mortality is mediated by the massive innate immune response or "the cytokine storm" (27). It is interesting to note that studies with the recreated $1918 \mathrm{H} 1 \mathrm{~N} 1$ virus in non-human primates have shown similar findings (26). Several of the viral proteins have been associated with the increased pathogenicity of these viruses (Fig. 1-2), most notably the HA. The presence of multiple basic amino acids at the cleavage site between the HA1 and HA2 subunits allows for the ubiquitous activation of the virus particles, which in turn result in enhanced virus dissemination and systemic infection $(8)$.

\section{Antigenic Structure of H5 HA}

The HA structure was solved over 25 years ago and showed the functional protein being a homotrimer consisting of a stalk and globular head (28). Stevens et al. have resolved the crystal structure of HA from A/Vietnam/1203/2004 (H5N1), an isolate from a fatal human infection (29). Although there are minor differences in the structure of HA of different subtypes, like the $\mathrm{H} 3$ protein, the major antigenic sites of H5 HA cluster in and around the receptor binding domain on the globular head (Fig. 1-3) (29). Generally, 
escape mutants of H5 HAs can be clustered into three hot spots (29); 1) the 140 loop (an exposed loop in the HA1 subunit extending from amino acid 140 to 145), 2) HA1 residues 156 and 157 and 3) HA1 129 to 133 (29).

\section{The Immune Response to Influenza Viruses}

Influenza A virus infection in humans is acute and the clearance of the virus is mediated by several parts of the immune system. Initially, influenza infection triggers a robust innate immune response in the form of the secretion of several cytokines and the massive influx of inflammatory immune cells such as neutrophils into the respiratory tract (7). The early clinical symptoms associated with influenza infection are caused by the innate immune responses. These early responses are essential not only for limiting virus replication but also for priming the virus specific B- and T-cell responses. On the other hand, influenza viruses have developed several evasion strategies such as encoding for the NS1 protein which, by sequestering viral RNA, blocks the triggering of the host interferon (IFN $\alpha / \beta)$ response (7).

\section{The Innate Immune Responses}

Our knowledge of the contribution of the innate immune system to the overall immune response to pathogens and vaccination has expanded over the last decade (3032). Recognition of conserved motifs that are associated with microbial infection is the initial signal for the generation of an appropriate innate and adaptive immune response (30-32). These motifs can be either derived from the invading microbes and thus called pathogen associated molecular patterns (PAMPs) or they can be endogenous danger signals caused by the infection (danger associated molecular patterns or DAMPs). The host cell sensors that evolved to recognize these PAMPs and DAMPs are known as the pattern recognition receptors or PRRs. Infection by influenza viruses can be detected by three major families of PRRs that were recently identified; the toll-like receptors (TLRs), the NOD-like receptors (NLRs) and the RIG-like receptors (RLRs) (30).

The Toll-Like Receptors (TLRs). To date, there are eleven TLRs (TLRs1-11) that have been characterized in mice (33). These receptors recognize a variety of ligands that are mostly microbial derived. TLRs mediate host innate immune responses by inducing the secretion of several proinflammatory cytokines through the activation of transcriptional factors such as NF- $\kappa \mathrm{B}, \mathrm{AP}-1$, ISRE-3 and ISRE-7 (33). TLR3 and TLR7 recognize double-stranded RNA (dsRNA) and single-stranded RNA (ssRNA), respectively (30). Therefore, both of these receptors were the most plausible candidates as receptors that detect influenza viruses (30). As for TLR3, studies with TLR3 ${ }^{-/}$mice and mice that are genetically deficient in TLR3 adapter molecule Toll/IL-1 domaincontaining adaptor-inducing IFN-beta (TRIF) have revealed that the adaptive immune responses to influenza viruses were intact (34). Type 1 interferon (IFN) response to influenza virus was, however, dependent on TLR7 signalling (35). 


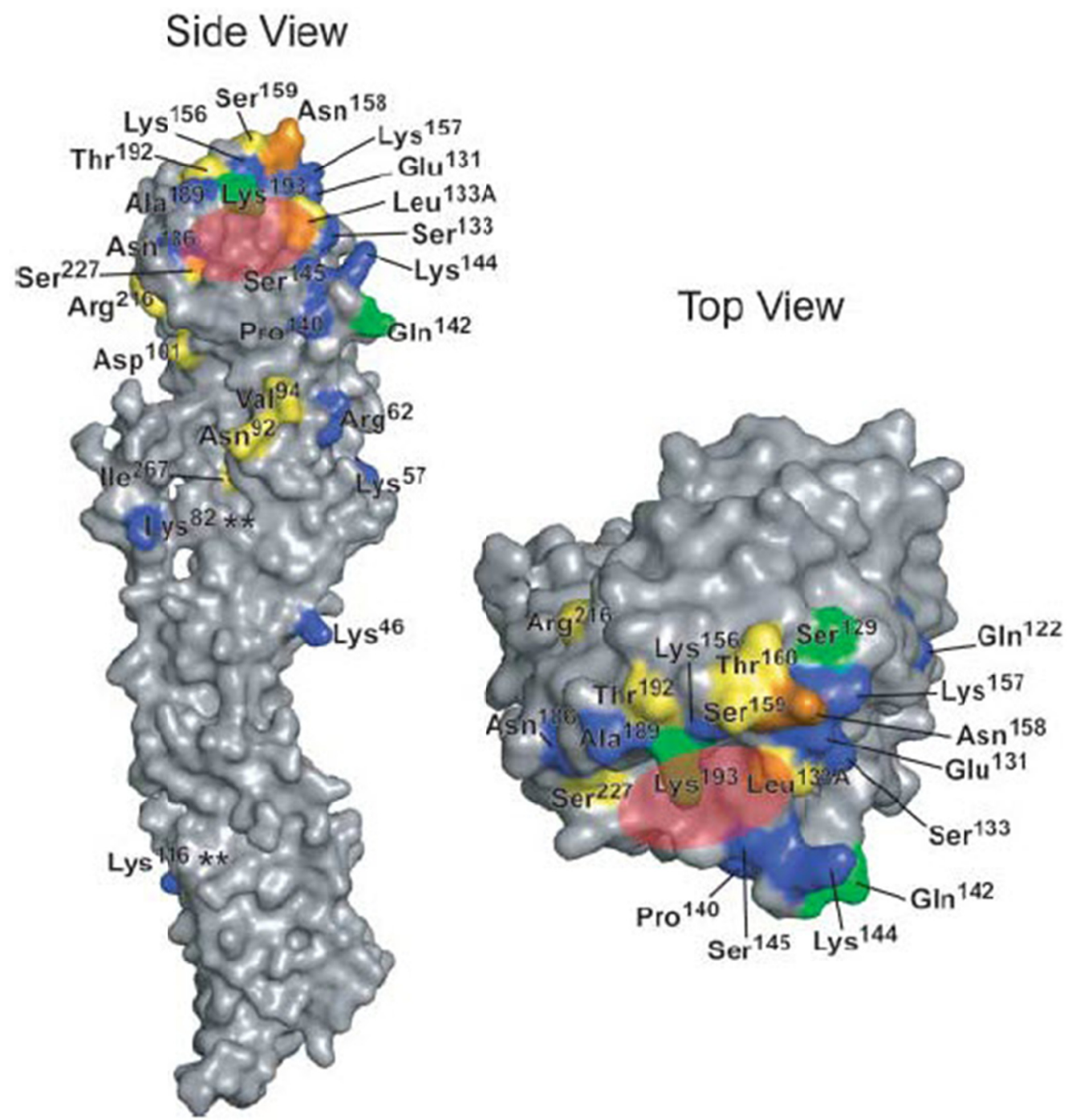

Figure 1-3. The antigenic structure of the H5 HA from A/Vietnam/1203/2004 (H5N1).

The natural mutations (identified by comparison to other 2004 and 2005 isolates) are colored blue while the escape mutants are colored yellow and the overlapping residues are green. The red oval represents the receptor binding domain and the orange residue is a newly introduced glycosylation site, reprinted with permission. J. Stevens et al., Science 312, 404 (Apr 21, 2006) (29). 
With the exception of TLR3 and a TRIF mediated arm of TLR4 signaling, all TLRs mediate their downstream signalling through an adapter molecule known as the myeloid differentiation primary response (MyD) 88 or MyD88. Similar to the findings with TLR7 signalling, IFN response to influenza viruses was also impaired in $\mathrm{MyD} 88^{-/-}$mice (Fig. 14) (33).

The NOD-Like Receptors (NLRs). NLRs were recently discovered as intracellular sensors that recognize pathogen-associated molecular patterns (PAMPs) as well as DAMPs (36). The prototypic members of this family, NOD1 and NOD2, recognize two distinct derivatives of bacterial peptidoglycans and mediate the subsequent cellular inflammatory response (36). There is a growing list of proteins that are being identified as members of the NLRs family (36). It is becoming increasingly clear that NLRs are essential components of the innate immune system not only in respect to the immunity to microbial pathogens but also to the pathogenesis of autoimmune diseases (36). Whereas NOD1 and NOD2 stimulation leads to activation of NF- $\kappa$ B and MAP kinases, other NLR proteins such as Ipaf, Nalp1, and NLRP3 mediate their actions through the activation of inflammatory caspases such as caspases 1 (36). Caspase-1, produced as a zymogen, is recruited to large multiprotein complexes known as "inflammasomes" through homotypic interactions with the adapter molecule ASC (36). ASC bridges the association of caspase-1 to NLR proteins such as Nalp1 and NLRP3 in the inflammasomes leading to the activation of caspase- 1 and the subsequent activation and secretion of the proinflammatory cytokines IL-1 $\beta$ and IL-18 (32). As for influenza infection, there are several reports that link increased susceptibility to influenza infection in mice that are deficient in NLRP3 (37). Thomas et al. showed that influenza RNA is detected by NLRP3 and the increased susceptibility was linked to the impaired wound healing in NLRP3 ${ }^{-/}$mice (37).

The RIG-Like Receptors (RLRs). RIG-I and MDA5, the prototypes of this family of receptors, were identified as cytosolic sensors of poly (I:C), a synthetic analogue of viral dsRNA (30). Their stimulation ultimately leads to type 1 IFN production through the activation of NF- $\kappa B$, IRF3 and IRF7 transcription factors (30). RIG-I was shown to detect influenza virus RNA and that genetic ablation of RIG-I caused increased susceptibility to influenza virus infection (30).

\section{The Humoral Immune Responses}

One of the most striking features of influenza virus infection is the speed with which the virus replicates once it is established in the respiratory epithelium. Preformed virus-specific antibodies in the serum or, preferably, on the airway mucosal surface can block viral entry and the subsequent establishment of infection (38). As a result, all licensed inactivated influenza vaccines to date are designed to generate an antibody response against the major virus surface molecules, HA and NA (39). A single infection with any strain of influenza viruses elicits lifelong antibody-mediated protection 


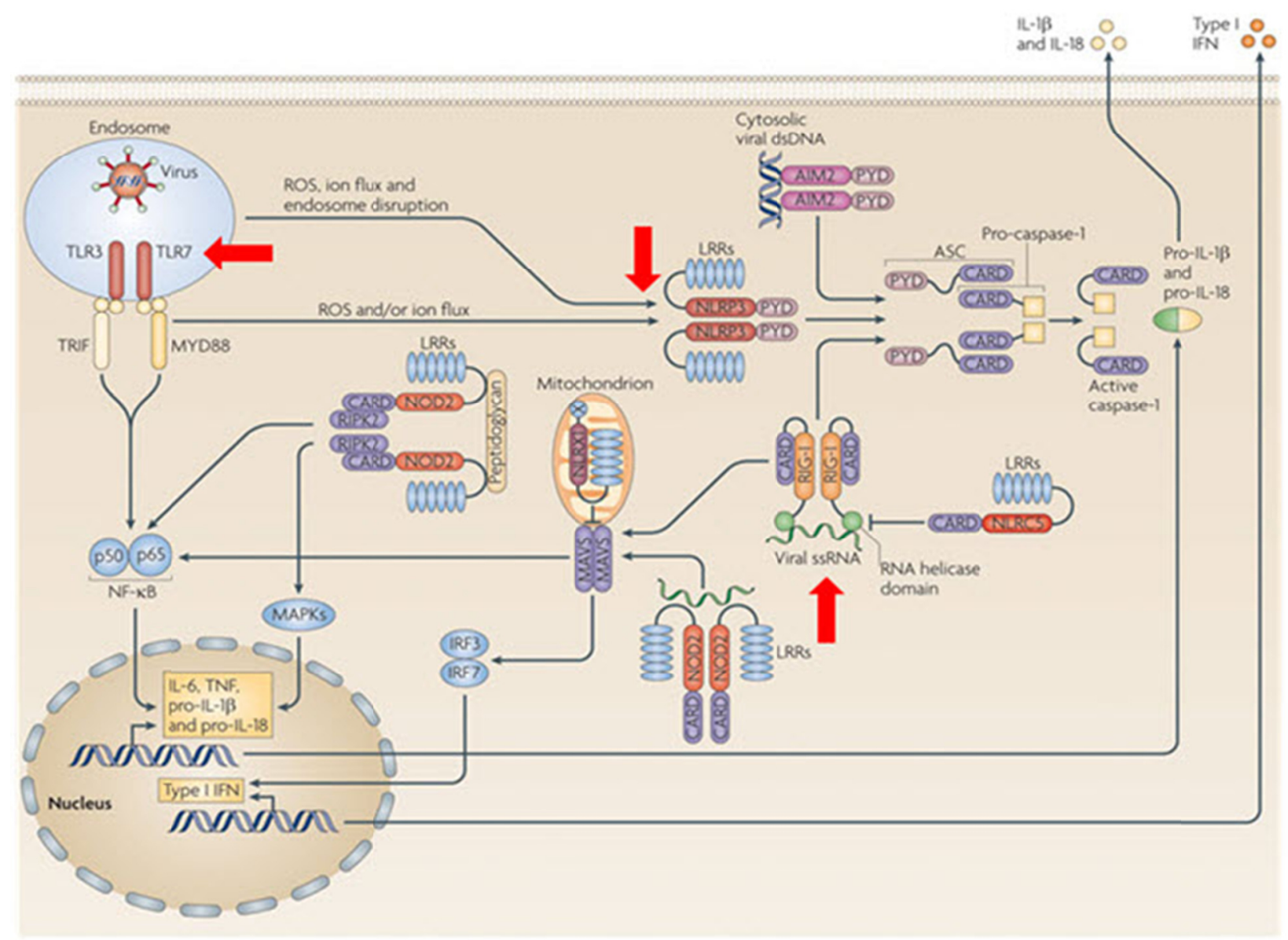

Figure 1-4. Innate immune pathways involved in recognition of viruses.

The three pathways that have been shown to be involved in sensing influenza virus infection are marked by the red arrows. Adapted with permission. T. D. Kanneganti, Nat Rev Immunol 10, 688 (Oct, 2010) (32). 
against homologous challenge (Fig. 1-5). This immunity is primarily mediated by the antibodies generated against the HA glycoprotein. Vaccination or infection-induced memory against a particular strain of influenza virus, however, does not guarantee protection against infection with other strains or even slightly drifted variants of the same strain (39).

Drifted strains are often generated within a single influenza season, a result of the persistent immune pressure driving frequent genetic and, consequently, antigenic variation allowing the virus to escape the immune response. This process is known as "antigenic drift" and it was recently proposed that along with the amino acid mutations that alter antigenicity, particularly within the HA, other mutations are introduced into the globular head of the HA to adjust receptor binding affinity (38). Therefore and despite annual flu vaccination programs, seasonal influenza infections cause a considerable amount of morbidity and mortality (11).

A more serious situation occurs when two influenza A viruses co-infect the same host cell, producing progeny virions that acquire HA molecules that have not been introduced widely into the human population (e.g. avian or swine HA), a phenomenon termed "antigenic shift" (13). A third potential viral immune evasion mechanism is acquisition of an HA molecule that had once circulated in the human population but ceased to circulate for decades. The human population has thus become mostly naïve to the reintroduced HA molecule. In either of these scenarios, the potential of emergence of a pandemic influenza virus increases especially if the newly reassortant virus acquires the ability to transmit among humans (13).

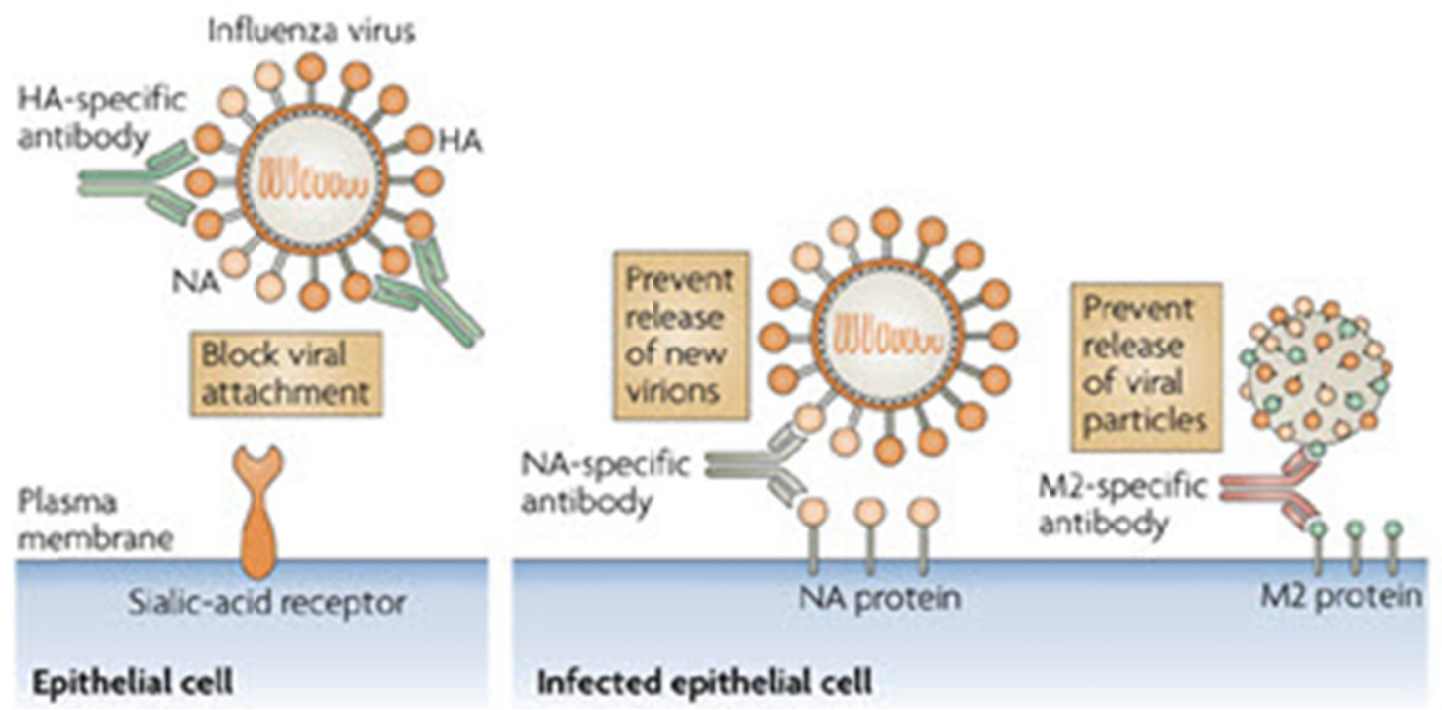

Figure 1-5. Mechanisms of antibody mediated immunity against influenza viruses.

Reprinted with permission. K. Subbarao, T. Joseph, Nat Rev Immunol 7, 267 (Apr, 2007) (8). 
Other viral surface glycoproteins that are also targeted by the antibody response are the NA and the matrix (M2) proteins. While I am focusing below on the challenges and recent advances in generating protective antibody responses to each of these viral targets, it is important to note that antibodies targeting some of the internal viral proteins such as the NP are also generated in the course of viral infection (40). However, the protective potential of such antibodies remains to be fully elucidated.

The Hemagglutinin (HA). As mentioned earlier, HA is the viral glycoprotein that mediates virus binding to lung epithelial cells leading to virus entry via receptormediated endocytosis. In addition, HA also mediates virus fusion with endosomal membrane, releasing viral genomic materials into the cytosol (41). This "invasion" role makes HA the primary target of protective antibodies generated by the host after infection or induced by influenza vaccines. Such antibodies, if present to sufficiently high titers, can neutralize the virus, i.e. completely prevent viral binding and invasion of airway epithelial cells achieving a "sterilizing immunity". Although there are several defined neutralizing antibody recognition sites the HA globular head and antibodies targeting one or more of these sites can efficiently neutralize virus infectivity, antigenic variation at all sites thwarts the development of long-term, protective influenza vaccines $(38,39)$. Thus, the challenge of eliciting broadly protective anti-HA antibodies is twofold; first the ability of such antibodies to cross-react with different HA subtypes and second, the ability to cross-react with escape variants of the original HA that the antibodies were raised against. Some have suggested that such broad specificities are unlikely to exist in principle, or repeated infection would have selected for them. However, recent studies have reported antibodies targeting the HA stem region that have broad neutralization potential across many subtypes (42). Additionally, the conservation of this region among different HA subtypes indicates certain structural restrictions that could limit antigenic escape (42). Nevertheless, the immunogenicity and protective potential of antibodies targeting the HA stem remain to be fully elucidated.

The Neuraminidase (NA). NA-specific antibodies are believed to interfere with the virus replication cycle, causing an overall "yield reduction" of virus infection (38, 39). Unlike anti-HA antibodies, NA-specific antibodies do not block infection initiation and so are not sterilizing, but can significantly reduce morbidity and mortality $(38,39)$. Similar to HA, NA exhibits a high degree of variability in terms of antigenicity in response to immune pressure rendering anti-NA- mediated protection rather inconsistent $(38,39)$. However, it was shown that antibodies elicited by human NA1 can partially protect against $\mathrm{H} 5 \mathrm{~N} 1$ infection (43).

The Matrix Protein 2 Ectodomain (M2e). A third viral trans-membrane protein antibody target is the matrix protein 2 (M2). Early studies in mice showed that anti-M2 antibodies provided partial protection (38). These results fuelled speculation that M2 might be the long sought target of a universal influenza vaccine, since, in comparison to HA and NA molecules, M2 proteins are highly conserved among different influenza 
subtypes (38). However, disappointingly, the anti-M2- mediated protection shown in mice has not been as dramatic in other, more relevant animal models (44).

\section{Cytotoxic T-Lymphocytes (CTLs)}

CTLs are essential for effective clearance of influenza virus infection (45). There are two key mechanisms via which CTLs help in controlling influenza virus infection. The first one is by direct killing of virus-infected cells. The second mechanism is through the production of various proinflammatory cytokines. In addition, the generation of virus specific memory CTLs significantly enhances virus clearance upon reexposure (45). The majority of CTL epitopes are derived from internal virus proteins such as NP and PB1 (45). These proteins are relatively conserved in comparison to HA, the main target of B-cell responses. Therefore, CTLs are important for generating a cross protective anti-influenza immune responses (46).

\section{Immune Correlates of Protection}

The immunogenicity of H5N1, and other influenza subtypes, virus vaccine is typically tested by the hemagglutination inhibition (HI) assay. For H5N1, horse red blood cells (RBCs) are used instead of the traditionally used turkey or chicken RBCs to enhance assay sensitivity (47). In general, the sensitivity and specificity of this assay is poor. Virus microneutralization ( $\mathrm{MN}$ ) assay is another assay that quantitatively measures the levels of neutralizing anti-HA antibodies and it is believed to be more sensitive and more functional than HI assay (47). HI titers of 40 or higher are generally considered protective assuming that the higher the HI or MN titers the higher the probability of protection (47). The enzyme-linked immunosorbent assay or ELISA has also been described to determine the levels of anti-HA antibodies (47). In ELISA, however, all (neutralizing and nonneutralizing) antibodies that bind to the H5 HA are measured. Although ELISA is rapid and more sensitive than $\mathrm{HI}$ and $\mathrm{MN}$, there is no information regarding the correlation between antibody levels and protection. Moreover, the use of whole virus preparations instead of purified HA as a coating antigens adds antibodies that target the internal protein such as anti-nucleoprotein antibodies to the equation. The clinical implications of non-neutralizing antibodies are not fully understood (47). Although these antibodies are more likely to be cross reactive among different HA subtypes, the mechanism(s) through which they may be contributing in viral clearance is not well studied.

\section{Influenza Vaccines}

\section{The Challenges of Vaccination against H5N1}

One of the main challenges facing influenza vaccination in general is the reliance of the production system on embryonated chickens eggs and the length of time between 
selection of vaccine strains and the availability of the first doses of formulated vaccines (Fig. 1-6) (48). Another key issue with the vaccine production (particularly H5N1 vaccines) process is that it is based on inactivation of live viruses that must be grown in bulk. Generation of bulk volumes of a novel virus, even reassortants with attenuating gene segments which are typically used, carries with it some safety concerns (48).

Influenza vaccines stimulate antibody responses that primarily target $\mathrm{HA}$ and as such are relatively specific towards the strain in the vaccine, i.e., the immunity generated is not particularly broad and their efficacy is largely dependent on the degree of matching between the vaccine and challenge strains. Because of the antigenic diversity encompassed by the avian reservoirs of H5N1 influenza A viruses, the possible emergence of a novel strain in humans, whether by nature or by design, poses a number of problems in terms of vaccine design (47). From a global health perspective, several factors must be considered to get maximum benefits from $\mathrm{H} 5 \mathrm{~N} 1$ vaccines: Their development and production must be quick; they should be affordable; they should elicit a broadly protective immune response; the vaccines should be immunogenic in populations of all ages preferably with a single dose and at a low antigen concentration; they should be reasonably stable and retain potency upon storage (47).

The major hurdle of preparing for an influenza outbreak is that the most effective vaccines against the virus promote an immune response against the protein that is most variable among viral protein, the HA. As discussed above, the HA is a surface glycoprotein that attaches and promotes entry of the virus into a host cell. As such, antibodies that can bind to the hemagglutinin can mediate neutralization of the virus. As discussed below, the HA is the mainstay of conventional influenza vaccine approaches.

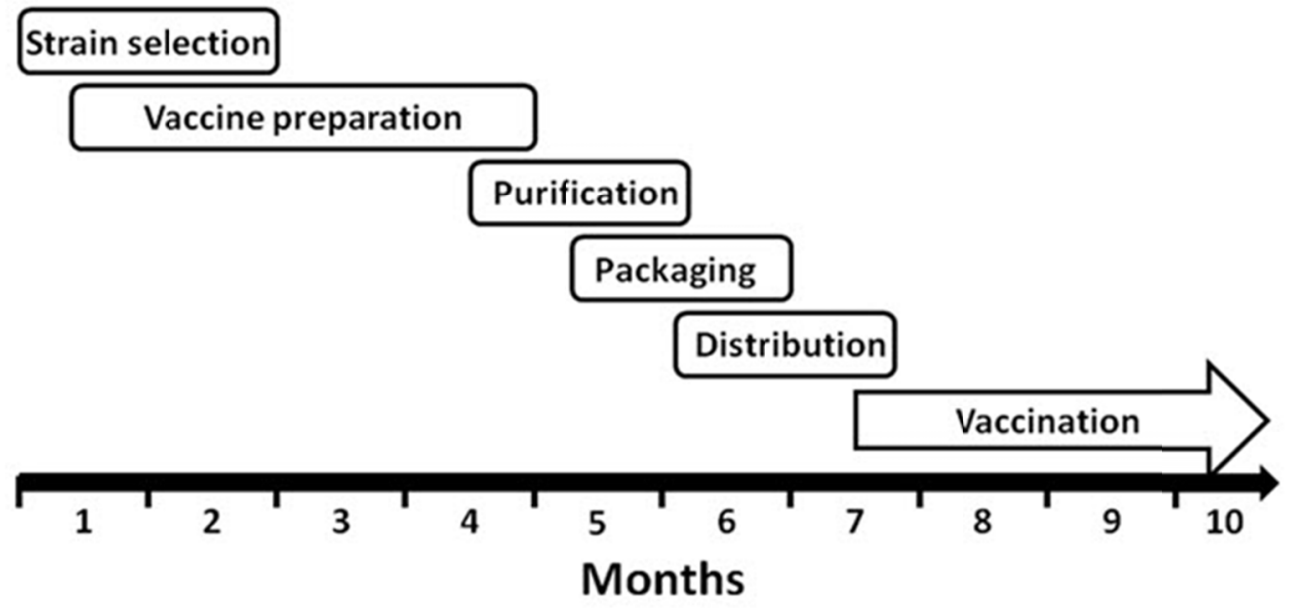

Figure 1-6. The estimated time line for the current influenza vaccine manufacturing process. 


\section{Inactivated Virus Vaccines}

Most of the currently licensed influenza vaccines are in the form of inactivated antigen preparations. As stated above, all vaccines work primarily through the generation of antibodies to HA. As such, they are potent, but prone to antigenic changes such as would be expected with the emergence of a new strain in a population. Clinical studies have shown that inactivated vaccines have an efficacy, measured by reducing serologically confirmed influenza illnesses, of 70\% in the age group between 14-60 years (49). The efficacy is reduced with both infants and the elderly (50). The first of the inactivated vaccine formulation is whole virion, the experimental use of which dates back to $1940 \mathrm{~s}$. The second is split virion which is derived by disrupting whole virus particles with disinfectants and finally, the subunit form, which is prepared by enriching for the viral surface glycoproteins HA and neuraminidase (NA) following disruption of viral particles. Although arguably more immunogenic, the reactogenicity associated with inactivated whole virus vaccine preparations, particularly in infants and children drove the development of the split vaccine technology back in the 1960s (51). Split and subunit vaccines have subsequently proven to be safe and have been delivered to millions of people (52). Unfortunately the relatively poor immunogenicity of split vaccines means that at least two doses of vaccine must be provided to generate protective immune responses in naïve individuals (53). In the event of a release of a novel and virulent strain of influenza, a two dose schedule for vaccination poses logistical and temporal issues.

\section{Live Attenuated Virus Vaccines}

Unlike inactivated vaccines, live attenuated influenza virus vaccines (LAIVs) are administered by intranasal inoculation of replication competent virus. In these vaccine viruses, the HA and NA of the target strain is introduced into a backbone of an attenuated, cold-adapted virus (54). Cold-adapted influenza viruses are attenuated by adapting the viruses to grow at $25^{\circ} \mathrm{C}(54)$. The first adapted influenza A virus (commonly referred to as the master strain) is A/Ann Arbor/6/60-H2N2 (54). The resulting vaccine virus has the antigenic phenotype of the target strain but the attenuated phenotype of the master strain.

The perceived advantages of the LAIV approach is that both a local immune neutralizing antibody and a cell mediated response can theoretically be generated. Cell mediated immunity is an attractive goal for influenza vaccines due to the fact that such immunity targets the more conserved viral proteins (46). In an ideal world, such immunity would protect across a range of different virus strains. In practice, in the few side by side comparisons of LAIV and inactivated vaccines in a seasonal influenza setting, the theoretical advantage of a broadened immune response is only seen in the younger cohorts (55). This is hypothesized to be due to the over attenuation of the viruses in an immunologically primed population. Within the context of a release of a novel strain, however, the population will essentially be naïve and LAIV could have an important role to play in terms of corresponding vaccine strategies. 
Despite some advantages, LAIV do suffer from some of the same issues as do their inactivated counterparts. LAIV will also likely require two doses to elicit optimal immune responses and their generation time is not substantially different from inactivated vaccines. LAIV also have some unique drawbacks in that it is possible not all HA and NA combinations will form viable viruses on the attenuated backbone, the vaccine virus must be able to infect the human upper respiratory tract (potentially an issue for avian strains), and potential safety risks of administering a live virus into a population before the target strain is widespread.

\section{Universal Target Approaches}

Due to the inherent variation of the influenza HA, a continuous quest of influenza immunologists has been to design influenza vaccines based on more conserved viral epitopes. One of the most studied and developed of these "universal target" antigen vaccines are those that target the extracellular portion of the M2 protein (M2e) (38). The M2 protein is a transmembrane ion channel and studies have shown that in appropriate configurations and in certain models, antibodies to the M2e domain can be protective; virtually no M2e antibodies are produced after natural infection.

M2e is not the only conserved epitope between influenza viruses and a number of other proteins have been targeted. These include nucleoprotein and polymerase proteins through $\mathrm{T}$ cell mediated approaches and also more conserved domains of HA (56). The immunogenicity of this part of the HA molecule and its protective potential is promising (42).

\section{Adjuvants for Vaccines against H5N1 Influenza Viruses}

\section{Why Are Adjuvants Needed for Influenza Vaccines?}

The poor immunogenicity of split or subunit influenza vaccines is a major challenge for the rapid response to an emerging virus. The impact is twofold, first higher quantities of antigen are needed per dose to elicit a protective immune response. Second, two doses will likely be needed for vaccine recipients to attain these protective responses. The use of adjuvants to overcome poor immunogenicity and for antigen sparing is not new to vaccinology, indeed, adjuvants such as alum salts for several human vaccines started several decades ago (Table 1-1) $(26,57)$. In addition to their ability to enhance vaccine immunogenicity, adjuvants such as the oil-in-water emulsion adjuvant MF59 have been shown to expand the breadth of an immune response to influenza vaccines (58, 59). MF59 induced the generation of higher serum antibody titers as well as more cross reactive responses when administered with split or subunit $\mathrm{H} 5 \mathrm{~N} 1$ vaccines $(58,59)$. Although these vaccines are unlikely to protect across subtypes, they do appear to be more cross reactive across the variations within a subtype. 
Table 1-1. Adjuvants that were shown to significantly boost influenza vaccines in humans.

\begin{tabular}{|c|c|c|c|}
\hline Adjuvant & Type & Mechanism of action & Licensed \\
\hline Aluminum salts or alum & Mineral salts & $\begin{array}{c}\text { Depot } \\
\text { NLRP3 inflammasome }\end{array}$ & Yes \\
\hline MF59 and AS03 & $\begin{array}{l}\text { Oil-in-water } \\
\text { emulsion }\end{array}$ & $\begin{array}{c}\text { Unknown } \\
\text { tissue inflammation }\end{array}$ & Yes \\
\hline $\begin{array}{c}\text { Cholera toxin }(\mathrm{CT}) \text { and } \\
\text { E.coli heat labile } \\
\text { enterotoxin }\end{array}$ & $\begin{array}{l}\text { Microbial } \\
\text { derived }\end{array}$ & $\begin{array}{l}\text { PRRs-independent } \\
\text { immune potentiation }\end{array}$ & No \\
\hline CpG & $\begin{array}{l}\text { Microbial } \\
\text { derived }\end{array}$ & Stimulation of TLR9 & No \\
\hline Muramyl tripeptide & $\begin{array}{l}\text { Microbial } \\
\text { derived }\end{array}$ & Stimulation of TLR2 & No \\
\hline IL-2 & Cytokine & $\begin{array}{l}\text { Direct stimulation of the } \\
\text { immune response }\end{array}$ & No \\
\hline
\end{tabular}

Adapted from R. L. Atmar, W. A. Keitel, Curr Top Microbiol Immunol 333, 323 (2009) (60).

\section{General Mechanisms of Action of Adjuvants}

Antigen presenting cells (APCs) such as dendritic cells (DCs) and macrophages are the main target immune cell population for most vaccine adjuvants (61). Once activated, APCs play a vital role in priming of antigen specific CD4 T-cells, the maestro of the adaptive immune response (61). Activation of APCs typically leads to upregulation of surface costimulatory molecules such as CD40 and CD80/86 mediate their interaction with CD4 T-cells (61). Naïve B-cells have the capacity to present antigens to CD4 T-cells as well. There are two broad categories of vaccine adjuvants based on their perceived mechanism of action. These two categories are delivery systems and immune potentiators (60). In both categories, proper activation of APCs and the subsequent adaptive immune response is the target.

Forming a depot of the vaccine antigen at the injection site and delaying antigen clearance are the leading hypotheses. In addition, the impartation of a particulate nature to vaccine antigens by these adjuvants is believed to increase antigen uptake by APCs. Regardless of the precise mechanism, improved antigen presentation by APCs seems to be the general outcome of using adjuvants as vaccine vehicles.

Immune potentiators are usually ligands for the pattern recognition receptors (PRRs) we previously discussed (see the innate immune section). The most characterized as vaccine adjuvants are the ligands for TLRs (60). Upon binding of such adjuvants to their specific PRRs, they initiate a signaling cascade that result in secretion of various proinflammatory cytokines and chemokines. These proinflammatory "messages" do not 
only activate APCs but also they dictate the type of the adaptive immune response to be activated as well (61). For example signaling through most TLRs leads to secretion of IFN-gamma and IL-12 which in turn favors the activation of specific subset of CD4 T-cells, the Th1 cells (61). The latter cells have been shown to provide the essential cytokines needed for proliferation of cytotoxic T-lymphocytes (CTLs) (61). Another subset of immune potentiators would be the direct administration of cytokines such as IL_2 in combination with the vaccine antigens $(60)$.

\section{Mineral Salts}

Mineral salts are the most commonly used vaccine adjuvants in humans (62). Insoluble aluminum salts or alum are the only adjuvant that is approved for use in human influenza vaccines in the US. Chemically alum composition is variable with aluminum oxyhydroxide and aluminum hydroxyphosphate being the most commonly used derivatives (63). The use of calcium derivatives has also been reported (63).

There are several mechanisms that have been proposed to explain alum adjuvanticity. Firstly, as a classic delivery system, alum is believed to form a depot of the vaccine antigen at the site of injection and to improve antigen uptake by APCs (61). Recently, several reports have shown that alum is capable of inducing a local inflammation at the injection site and this inflammation is correlated with its immune enhancing effects (61). These reports have revealed that alum mediates NLRP3 inflammasome activation and the consequential release of the proinflammatory cytokines IL-1 beta and IL-18 (64). Whether the deletion of this pathway significantly impacts the adjuvant effects of alum is still controversial (65-67).

Alum has been used with various forms of influenza vaccines for decades (60). When used with subunit H5N1 influenza vaccines, the results were inconsistent. While Bresson et al. observed a greater response to the adjuvanted vaccine formulation; this was only at the highest dose $(30 \mu \mathrm{g})$ of the vaccine (53). Keitel et al. observed more frequent seroconversion responses with the adjuvanted vaccine at the $7.5 \mu \mathrm{g}$ dose group with no significant differences among other groups (68). Additional studies found no evidence for a significant adjuvant effect of alum (69). Overall evaluation of alum-adjuvanted subunit $\mathrm{H} 5 \mathrm{~N} 1$ influenza vaccines in humans indicates that alum effect is modest. This is equally true for whole virus influenza vaccines as well (70).

\section{Emulsions}

Emulsions are the second most commonly used adjuvants in human vaccines. Emulsions are prepared by mixing two immiscible liquid phases (aqueous and oily phases) in the presence of surfactants. There are two types of emulsions based on the nature of the dispersed (or inner) vs. the dispersing (or outer) liquid phase; water-in-oil (w/o) and oil-in-water (o/w) emulsions. Despite being efficient adjuvants, w/o emulsion adjuvants such as complete Freund's adjuvant (CFA) are no longer used in human 
vaccines due to their high reactogenicity (62). On the other hand, oil-in-water emulsions such as MF59 and AS03 have been shown to be effective and safer adjuvants (60).

The mechanism of action of emulsion adjuvants is unknown. Initially it was thought that similar to other vaccine vehicles, emulsions form a depot of the vaccine antigen allowing its slow release from the injection site. However, studies with labeled gD2 from type 2 herpes simplex virus (HSV) revealed similar release kinetics between the unadjuvanted and the MF59 adjuvanted antigen (71). Similarly to alum, it is proposed that MF59 improve antigen uptake by APCs (72). More recently studies with MF59 adjuvanted vaccines have shown that MF59 stimulates a potent localized inflammatory response at the site of injection (73). Unlike alum, the innate immune pathway(s) involved in emulsion-based adjuvants induced inflammation is not clear.

\section{MF59}

Among the variety of $\mathrm{o} / \mathrm{w}$ emulsion based adjuvants that have been tested with influenza vaccines, MF59 is the most extensively studied (74). The oil phase is comprised of $4.3 \% \mathrm{w} / \mathrm{v}$ squalene oil. Two surfactants were used; Tween 80 (polyoxyethylene sorbitan monooleate) and Span-85 (sorbitan trioleate). Sodium citrate buffer $(10 \mathrm{nM})$ is included to improve the emulsion stability (74). In 1997, MF59 has been licensed in combination with inactivated influenza vaccine as a seasonal vaccine for the elderly in Europe (74).

Initial trials of MF59 with H5 vaccines were performed with the inactivated H5N3 whole virus vaccine (75). These studies demonstrated that MF59 had significantly boosted the antibody response in comparison to the unadjuvanted vaccine. Similar results were reported by Atmar et al. using MF59 in combination with an inactivated influenza vaccine derived from H9N2 avian influenza A viruses (76). These initial studies have not only demonstrated the ability of MF59 to significantly improve the antibody response to influenza vaccines but also its dose sparing capacity. In a comprehensive clinical trial, Bernstein et al. showed that MF59 in combination with a subunit H5N1 influenza vaccine derived from A/Vietnam/1203/2004 induced more potent anti-H5 antibody responses in comparison to the alum adjuvanted or the unadjuvanted formulations (69). These results strongly suggest that unless novel production and/or adjuvant systems are developed, oil-in-water adjuvants should go hand-in-hand with the development of vaccines for potential influenza pandemics.

\section{Scope and Objectives of Dissertation}

An ideal pre-pandemic H5N1 vaccine formulation should be able to elicit a fast and protective antibody response preferably after a single immunization. It also should prime a diverse repertoire of $\mathrm{H} 5$ specific B cells capable of responding to subsequent challenge with as many viral antigenic variants as possible. Preliminary studies have shown that the use of the oil-in-water emulsion based adjuvant MF59 in combination 
with subunit $\mathrm{H} 5 \mathrm{~N} 1$ influenza vaccines has significantly enhanced the overall immune responses not only to the vaccine parent viral strain but also to drifted strains as well. However, it remained unclear whether this adjuvant-mediated broadening of the antibody responses are resulting from a real expansion of the targeted epitopes within the vaccine antigen or due to a general enhancement of the antibody titers, which in turn lead to the detection of minor responses that were otherwise undetectable. Considering the importance of adjuvants' impact on the kinetics and the broadness of the protective antibody responses, it is surprising that we understand little about the factors involved. Finally, determining the underlying mechanisms that mediate adjuvant effects allows for the rational design of new adjuvants, which in turn can be utilized to enhance adjuvants' overall efficacy.

\section{Specific Aim 1}

To determine, in a ferret model, how soon following a single immunization with the prepandemic $\mathrm{H} 5 \mathrm{~N} 1$ vaccine protection against lethality can be attained and how adjuvants would affect the kinetics of such protection. We immunized ferrets with a single dose of the H5N1 subunit vaccine either unadjuvanted or adjuvanted with MF59 or alum at different time points prior to challenge with a lethal dose of the homologous H5N1 virus.

\section{Specific Aim 2}

To determine whether MF59 afford qualitative or simply quantitative changes in a vaccine induced antibody response. Although a number of studies have addressed these issues at the level of antibody quantity, little is known about the quality of these responses. More specifically, we wanted to determine how the priming environment affects the breadth of the antibody responses to HA in the murine model.

\section{Specific Aim 3}

To determine the underlying molecular mechanisms that mediate adjuvant effects of oil-in-water emulsion adjuvants. We examined the role of the NLRP3 inflammasome in the mediating the antibody responses to MF59-adjuvanted $\mathrm{H} 5 \mathrm{~N} 1$ vaccines. 


\section{CHAPTER 2. ADJUVANTS ENHANCE THE KINETICS OF PROTECTIVE ANTIBODY RESPONSES TO H5N1 INFLUENZA VACCINES}

\section{Introduction}

Highly pathogenic avian influenza A (HPAI) viruses are pathogens to which most of the human population is immunologically naïve (77). Since 1997, when the first direct transmission of the highly pathogenic $\mathrm{H} 5 \mathrm{~N} 1$ influenza viruses from birds to humans was identified in south East Asia, the viruses continued to circulate in birds causing increasing number of infections and death (77). From the clinical point of view, the main difference between seasonal and HPAI viruses' infection is the severity of the clinical symptoms associated with the latter infection (22). These symptoms typically include pneumonia with progressive respiratory failure, gastrointestinal symptoms and liver and renal dysfunction (22). Central nervous system involvement has also been reported leading to an overall mortality rate of $60 \%(22)$.

Vaccination is the most efficient way of controlling influenza and influenzarelated complications. The 1997 and 2003 H5N1 outbreaks have encouraged the World Health Organization (WHO) to develop and stockpile prepandemic H5N1 vaccines (http://www.who.int/csr/disease/avian influenza/guidelines/H5VaccineVirusUpdate2008 0214.pdf). These vaccines are to be deployed during the early phases of a pandemic while a matching vaccine is developed. A major hurdle in the prepandemic preparedness plan is that the vaccine manufacturing capacity does not meet the demand. Moreover and for yet unclear reasons, subunit vaccines derived from avian influenza viruses have been shown to be poorly immunogenic (8). Clinical trials with subunit $\mathrm{H} 5 \mathrm{~N} 1$ vaccines have shown that two immunizations containing high amounts of antigen $(90 \mu \mathrm{g}$ of hemagglutinin or HA) were required to elicit what is considered to be a protective antibody response in $50 \%$ of the study subjects (78). Therefore, the use of adjuvanted influenza vaccines was recommended.

The use of adjuvants to boost influenza vaccines immunogenicity was introduced several decades ago (62). A vital advantage of using adjuvants is the ability, using smaller quantities of the vaccine antigens, to elicit antibody responses that is equivalent those generated using higher quantities of the unadjuvanted vaccine (60). This antigen sparing capacity is particularly important in the case of avian influenza vaccines because it will allow for broader vaccine coverage. The addition of alum to subunit H5N1 influenza vaccines has induced only marginal improvements (79). However, the oil-in-water emulsion adjuvant MF59 showed more promising results; higher serum antibody titers as well as more cross reactive responses when. MF59 is licensed for use in seasonal influenza vaccines in Europe (60). MF59 was tested with H5 and H9 vaccines and showed promising results in terms of potentiating the immune response with evidence of increasing cross reactivity with antigenic variants as well (76). These studies strongly suggest that unless novel production and/or adjuvant systems are developed, oilin-water adjuvants will become a critical part of pandemic influenza preparedness and influenza vaccines in general. 
Most of the H5N1 vaccine trials, either clinical or preclinical, have tested the ability of different vaccine formulations to either induce a serologically protective antibody levels or protect vaccinated animals against challenge-mediated mortality three to four weeks after the boosting dose. While such information is essential in assessing the overall efficacy of the vaccines, in the case of an avian influenza outbreak health care workers and individuals involved in outbreak disease control and eradication activities will have to engage almost immediately. The Center for Disease Control and Prevention (CDC) is recommending that such personnel, in addition to receiving prophylactic antiviral drugs, should also be vaccinated with the seasonal influenza vaccines to reduce the probability of a co-infection with both human and avian influenza (http://www.cdc.gov/flu/avian/professional/infect-control.htm). But seasonal vaccines will unlikely provide protection against human infection with the avian viruses. Therefore, once an outbreak or a pandemic of H5N1 is declared, vaccinating those individuals and other high risk groups with a single dose of the pre-pandemic vaccine might be beneficial. Indeed, Middleton et al. found that a single dose of adjuvanted H5N1 vaccines when administered 4 weeks prior to challenge with $\mathrm{H} 5 \mathrm{~N} 1$ viruses was able to protect ferrets against lethality (80). However, the pre-incubation period between administering the vaccine and attaining protection was not determined; yet it is a key factor in understanding the full utilization of a prepandemic vaccine (80).

The major aim of the present study was to determine, in a ferret model, how soon following a single immunization with the prepandemic $\mathrm{H} 5 \mathrm{~N} 1$ vaccine protection against lethality can be attained and how adjuvants would affect the kinetics of such protection. We found that as early as 7 days after immunization with the MF59-adjuvanted vaccine, more than $50 \%$ of the ferrets survived a highly lethal challenge. We then confirmed our results using alum, a different adjuvant system. We believe that these encouraging results with the adjuvanted $\mathrm{H} 5 \mathrm{~N} 1$ vaccines are important in the context of planning various strategies to mitigate the early events of a potential H5N1 pandemic.

\section{Materials and Methods}

\section{Viruses}

The influenza A/Vietnam/1203/04 (H5N1) virus was obtained from the World Health Organization (WHO) collaborating laboratories. Stock virus was grown in the allantoic cavities of 9 days old embryonated chicken eggs for $32 \mathrm{~h}$ at $36^{\circ} \mathrm{C}$, and aliquots were stored at $-70^{\circ} \mathrm{C}$ until used. Virus titer was determined by calculating the EID $_{50}$. Experiments with highly pathogenic H5N1 viruses were conducted in a biosafety level 3+ containment facility under applicable laws and guidelines. 


\section{Immunization and Challenge}

Female ferrets 3 months of age and seronegative for currently circulating influenza A H1N1, H3N2, and influenza B viruses were obtained from Triple F farms (Sayre, PA). Ferrets (3-6 per group) were immunized by intramuscular injection of a single $15 \mu \mathrm{g} \mathrm{HA} /$ dose of the pre-pandemic H5N1 vaccine derived from A/Vietnam/1203/04 (H5N1) either unadjuvanted or in combination with MF59 or alum. At several time points $(4,7,14$ or 21 days) post immunization, ferrets were challenged by intranasal inoculation with $10^{6} 50 \%$ egg infectious doses $\left(\mathrm{EID}_{50}\right)$ of the highly pathogenic A/Vietnam/1203/2004 (H5N1) influenza virus. For the inoculation, ferrets were anesthetized with isofluorane and then inoculated intranasally in a total volume of $1 \mathrm{ml}$. All animal experiments were approved by the Animal Care and Use Committee of St. Jude and performed in compliance with relevant institutional policies of the National Institutes of Health regulation and the Animal Welfare Act. For the next 10 days following challenge, animals were closely monitored for clinical signs of infection. Animals that developed severe clinical signs in terms of weight loss, lethargy or neurological symptoms were euthanized for humane reasons.

\section{Virus Titration}

At selected time points after virus inoculation, ferrets were anesthetized with ketamine $(25 \mathrm{mg} / \mathrm{kg})$, and $0.5 \mathrm{ml}$ of PBS with antibiotics was slowly introduced into each nostril, recovered, measured, and brought to a volume of $1.0 \mathrm{ml}$ with sterile PBS containing antibiotics. Bovine serum albumin $7.5 \%$ was added at a ratio of $1: 20(\mathrm{v} / \mathrm{v})$ as a stabilizing agent. Virus was titrated in embryonated chicken eggs and expressed as $\log _{10}$ EID $_{50}$ per milliliter. The limit of virus detection was $<0.75 \log _{10} \mathrm{TCID}_{50} / \mathrm{ml}$.

\section{Serologic Tests}

Ferret sera were obtained prior to challenge and were treated with receptor destroying enzyme (Accurate Chemicals and Scientific, New York) overnight at $37^{\circ} \mathrm{C}$, heat-inactivated at $56^{\circ} \mathrm{C}$ for $30 \mathrm{~min}$, and diluted 1:10 in PBS. Sera were tested by the hemagglutination assay (HI) against the homologous A/Vietnam/1203/2004 (H5N1) virus using $1.0 \%$ horse red blood cells. For antigen-specific ELISA, microtiter plates (Corning, Lowell, MA) were coated overnight at $4^{\circ} \mathrm{C}$ with the recombinant $\mathrm{H} 5 \mathrm{HA}$ protein from A/Vietnam/1203/2004 (eEnzyme, Montgomery Village, MD) in PBS. After an overnight incubation with serial dilutions of ferrets' sera, H5-specific IgG antibodies were detected with a goat anti-ferret IgG alkaline-phosphatase conjugate (Biotrend, Cologne, Germany) diluted 1:1000 in PBS with 1\% bovine serum albumin (BSA). The substrate ( $p$ nitrophenyl phosphate; Sigma-Aldrich, Atlanta, GA) was added, plates were incubated for $30 \mathrm{~min}$ at room temperature for color development, and OD values were determined at $405 \mathrm{~nm}$ in an ELISA reader (Biorad, Los Angeles, CA). 


\section{Results}

\section{MF59-Adjuvanted H5N1 Vaccine Provides Fast Protection against Challenge- Associated Mortality and Morbidity}

We examined the protective effect of single dose (15 $\mu \mathrm{g} \mathrm{HA} /$ dose $)$ immunization with either the unadjuvanted prepandemic H5N1 subunit vaccine derived from A/Vietnam/1203/04 (H5N1) or adjuvanted with MF59. Immunizations were given at 3 different time points $(14,7$, or 4 days) prior to challenge with a high dose of the homologous virus, A/Vietnam/1203/04 (H5N1) (Table 2-1). For ferrets that received the unadjuvanted vaccine 4 days (V4), 7 days (V7) or 14 days (V14) prior to challenge, we observed severe clinical symptoms including weight loss, anorexia, neurological signs, extreme lethargy, and they all died or had to be euthanized by day 5 post challenge (Table 2-1 and Fig. 2-1). Ferrets given the MF59-adjuvanted vaccine 4 days (M4) prior to challenge showed similar symptoms to those that received the unadjuvanted vaccine and they all died or had to be euthanized by day 5 post challenge for humane reasons (Table 2-1). Although four out of the six ferrets that received the MF59-adjuvanted immunization 7 days prior to challenge survived, they showed typical symptoms of influenza virus infection including weight loss and mild lethargy (Table 2-1 and Fig. 2-1). The clinical symptoms were, however, milder than those observed with ferrets that received the unadjuvanted vaccine or the M4 group. Two out the six ferrets in the M7 group developed neurological symptoms and were euthanized 5 and 7 days post challenge (Table 2-1). All ferrets given the MF59-adjuvanted vaccine 14 days prior to challenge (M14) survived and showed minor clinical signs (Table 2-1 and Fig. 2-1). These data show that a protective response can be generated in as little as 7 days following immunization with a single dose of the adjuvanted subvirion $\mathrm{H} 5 \mathrm{~N} 1$ influenza vaccine.

\section{Kinetics of the Antibody Response to the MF59-Adjuvanted H5N1 Vaccines}

We tested the induction of H5 specific antibodies in the ferrets' sera prior to challenge. Not surprisingly, ferrets that received the unadjuvanted vaccine (V4, V7 and V14 groups) failed to develop a detectable H5 specific antibody titer measured either by hemagglutination inhibition (HI) or ELISA (Table 2-2). Similarly, we failed to detect H5 specific antibodies in sera collected from M4 group (Table 2-2). Although four out of the six ferrets that were immunized with the MF59-adjuvanted vaccine 7 days prior to challenge survived, we also failed to detect H5 specific titers these animals' sera (Table 2-2). Expectedly, H5 specific titers were detectable in sera of the ferrets that belonged to M14 group by HI and ELISA titers of 16.6 and 300, respectively (Table 2-2). These data show that the presence of detectable antibody titers is predictive of protection but also that a lack of detectable titers as measured by HI or ELISA is not necessarily predictive of death. To assess the ability of the different immunization regimens to prevent virus replication in the upper respiratory tract of ferrets, we determined viral titers in nasal wash samples on day 5 post inoculation (Table 2-2). 
Table 2-1. Effect of the different vaccination regimen on survival and clinical signs of infection in ferrets challenged with A/Vietnam/1203/04 (H5N1) influenza virus.

\begin{tabular}{|c|c|c|c|c|c|c|}
\hline \multirow{2}{*}{$\begin{array}{l}\text { Group } \\
\text { name }\end{array}$} & \multirow[b]{2}{*}{$\begin{array}{l}\text { Vaccination regimen (days } \\
\text { prior to challenge) }\end{array}$} & \multirow{2}{*}{$\begin{array}{c}\text { No. } \\
\text { dead/ } \\
\text { total no. }\end{array}$} & \multirow{2}{*}{$\begin{array}{c}\text { Time of death, } \\
\text { days after } \\
\text { infection }\end{array}$} & \multicolumn{3}{|c|}{ Clinical signs $^{b}$} \\
\hline & & & & $\begin{array}{l}\text { Respiratory } \\
\text { symptoms }\end{array}$ & $\begin{array}{l}\text { Neurological } \\
\text { symptoms }\end{array}$ & $\begin{array}{l}\text { Lethargy } \\
\text { (RII) }\end{array}$ \\
\hline V4 & $15 \mu \mathrm{g}$ H5N1 Vaccine (4) & $3 / 3$ & $4,4,5$ & $3 / 3$ & $2 / 3$ & 3.0 \\
\hline V7 & 15 g H5N1 Vaccine (7) & $3 / 3$ & $4,5,5$ & $2 / 3$ & $2 / 3$ & 2.5 \\
\hline V14 & $15 \mu \mathrm{g}$ H5N1 Vaccine (14) & $3 / 3$ & $5,5,5$ & $2 / 3$ & $2 / 3$ & 3.0 \\
\hline M4 & $\begin{array}{c}15 \mu \mathrm{g} \text { H5N1 Vaccine }+ \text { MF59 } \\
\text { (4) }\end{array}$ & $3 / 3$ & $3,4,5$ & $3 / 3$ & $3 / 3$ & 3.0 \\
\hline M7 & $\begin{array}{c}15 \mu \mathrm{g} \text { H5N1 Vaccine }+ \text { MF59 } \\
\text { (7) }\end{array}$ & $2 / 6$ & 5,7 & $3 / 6$ & $2 / 6$ & 1.5 \\
\hline M14 & $\begin{array}{c}15 \mu \mathrm{g} \text { H5N1 Vaccine + MF59 } \\
(14)\end{array}$ & $0 / 5$ & - & $0 / 5$ & $0 / 5$ & 0.0 \\
\hline
\end{tabular}

${ }^{\text {a }}$ Ferrets were immunized with a single $15 \mu \mathrm{g}$ dose of the H5N1 SU vaccine derived from A/Vietnam/1203/04 (H5N1) with or without MF59. After the indicated number of days after immunization, ferrets were challenged by inoculation with $10^{6}$ EID $_{50}$ of $\mathrm{A} / \mathrm{Vietnam} / 1203 / 04$ virus.

${ }^{\mathrm{b}}$ Clinical signs were observed for 10 days after virus inoculation. Except for lethargy and weight loss, findings for clinical signs are given as no. of ferrets with sign/total no. Respiratory signs included sneezing, wheezing, and nasal discharge; neurological signs included hind limb paresis, ataxia, torticollis, and tremors.

${ }^{\mathrm{c}}$ The relative inactivity index (RII) is scored as follows: 0 , alert and playful; 1 , alert, playful when stimulated; 2 , alert but not playful when stimulated; 3 , neither alert nor playful when stimulated (81). 


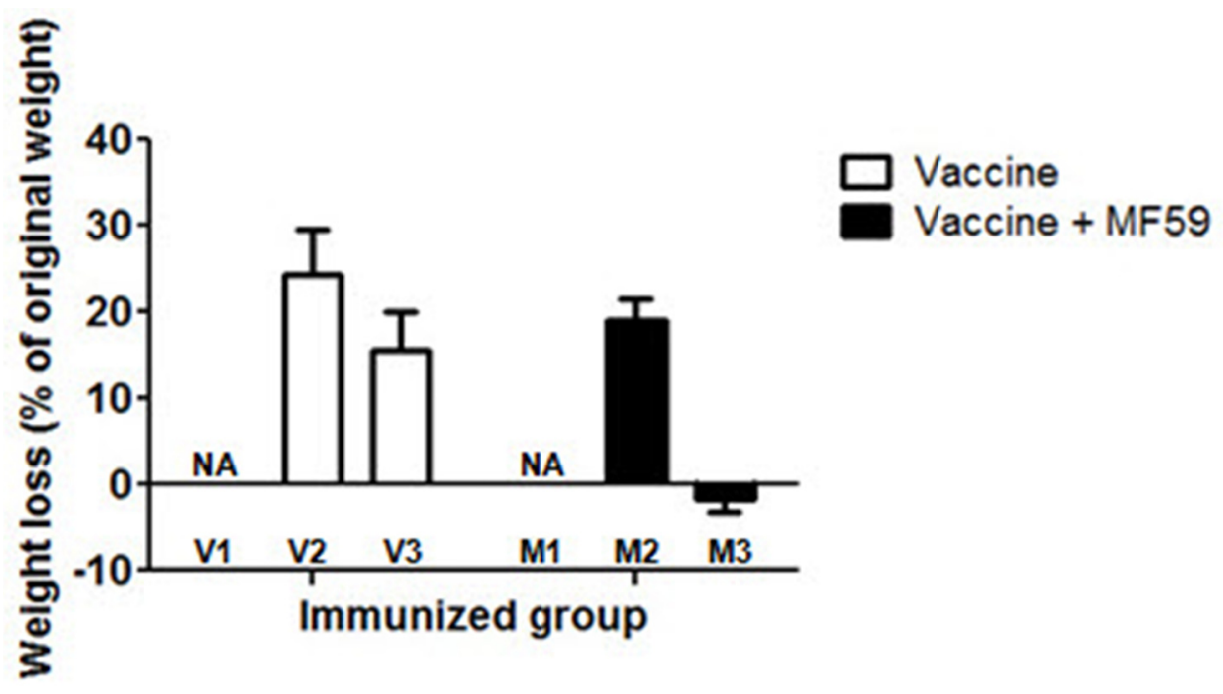

Figure 2-1. Weight loss in the unadjuvanted and MF59-adjuvanted groups 5 days post challenge with A/Vietnam/1203/04 (H5N1) virus.

Weight loss was calculated as the mean percentage of weight loss 5 days post challenge (as compared to weight at challenge day). NA, not applicable. Error bars indicate standard error. 
Table 2-2. Effect of different vaccination regimen on A/Vietnam/1203/04 (H5N1) influenza virus replication in the upper respiratory tract of ferrets.

\begin{tabular}{ccccc}
\hline Group $^{\mathbf{a}}$ & HI titer & $\begin{array}{c}\text { ELISA titer } \\
\text { (IgG) }\end{array}$ & $\begin{array}{c}\text { No. shedding/ total no. of } \\
\text { ferrets tested }^{\mathbf{b}}\end{array}$ & Virus titer $^{\mathbf{c}}$ \\
\hline V4 & $<$ & $<$ & $\mathrm{NA}^{\mathrm{d}}$ & $\mathrm{NA}$ \\
V7 & $<$ & $<$ & $2 / 3$ & $4.3( \pm 0.35)$ \\
V14 & $<$ & $<$ & $3 / 3$ & $4.5( \pm 0.25)$ \\
M4 & $<$ & $<$ & $\mathrm{NA}$ & $\mathrm{NA}$ \\
M7 & $<$ & $<$ & $3 / 3$ & $4.0( \pm 0.38)$ \\
M14 & 16.6 & 300 & $1 / 3$ & 2.5 \\
\hline
\end{tabular}

${ }^{\text {a }}$ Vaccination regimen for these groups is shown in Table 2-1.

${ }^{\mathrm{b}}$ Based on the viral titers measured in the nasal washes collected 5 days post challenge with $10^{6} \mathrm{EID}_{50}$ of A/Vietnam/1203/04 virus. The assay detection limit was $0.75 \log _{10}$ $\mathrm{EID}_{50} / \mathrm{mL}$.

${ }^{c}$ Titers (from day 5 nasal washes and expressed as $\log _{10} \mathrm{EID}_{50} / \mathrm{mL}$ ) are given as means \pm $\mathrm{SD}$, unless only 1 animal was shedding virus.

${ }^{\mathrm{d}} \mathrm{NA}$, not applicable, only one animal from these groups survived to day 5 . 
All ferrets that were immunized with the unadjuvanted vaccine and survived to day 5 shed virus at high titers (4.3 and $4.5 \log _{10} \mathrm{EID}_{50} / \mathrm{mL}$ for V7 and V14 groups respectively). $\mathrm{M} 7$ ferrets shed virus to similar extent $\left(4.0 \log _{10} \mathrm{EID}_{50} / \mathrm{mL}\right)$. Only one ferret from the M14 group was shedding detectable viral titer at day 5 post challenge (Table 2-2). The virus titer shed by this animal was $1.5 \operatorname{logs}$ less than virus titers shed by ferrets that belonged to the unadjuvanted vaccine (V7 and V14) or M7 groups (2.5 $\log _{10}$ $\mathrm{EID}_{50} / \mathrm{mL}$, Table 2-2).

\section{Impact of Alum on H5N1 Vaccine-Mediated Protection Kinetics}

We next assessed whether a similarly early protective response could be generated after a single immunization with the prepandemic $\mathrm{H} 5 \mathrm{~N} 1$ vaccine combined with a different adjuvant. Similar to MF59, alum has been licensed to be used in combination with human influenza vaccines. To this end, we used the same single immunization followed by challenge approach to test whether alum-adjuvanted H5N1 vaccine can provide the early protection that we observed with the MF59-adjuvnated vaccine. Based on the assumption made from clinical trials that alum would be inferior to MF59 in adjuvant activity, immunizations were given at three different time points (21, 14 and 7 days) prior to challenge. We used an immunization dose of $15 \mu \mathrm{g} \mathrm{HA} /$ dose. As shown in Table 2-3, ferrets belonging to the three groups (A7, A14 and A21) survived the lethal challenge. However, ferrets that received the immunization seven days prior to challenge or A7 group suffered a modest weight loss (11.1\%) and 2 out of 3 ferrets showed respiratory signs of infection in addition to be mildly lethargic (Table 2-3).

Similarly to what was observed with MF59-adjuvanted vaccine, A14 and A21 groups showed minimal clinical symptoms after challenge with no weight loss by day 5 post challenge (Table 2-3). This lack of challenge associated morbidity in the A14 and A21 groups correlated well with the development of detectable H5 specific HI (23.3 and 46.6, respectively) and $\operatorname{IgG}$ (900 and 1140, respectively) titers prior to challenge (Table 2-4). It also correlated with the lack of detectable viral titers in the nasal washes collected from these ferrets 5 days post challenge (Table 2-4). For A7 ferrets, similar to surviving ferrets in the M7 group, there were no detectable anti-H5 antibody titers prior to challenge (Table 2-4). However and consistent with the milder clinical symptoms observed in A7 compared to M7 ferrets, 2 out of the 3 ferrets in A7 group cleared the virus by day 5 post challenge (Table 2-4). From these results it is possible to conclude that in the H5N1 ferret model, alum is as effective, if not more so, as MF59 in inducing a rapid protective immune response when combined with $\mathrm{H} 5 \mathrm{~N} 1$ prepandemic vaccines.

\section{Discussion}

Preventive vaccination, antiviral drugs and non-pharmaceutical interventions (such as school closure) are the main public health measures needed to contain an influenza pandemic. The major drawback of anti-influenza antiviral drugs (M2 ion channel blockers and NA inhibitors) is the development of drug resistance mutants and 
Table 2-3. Impact of Alum-adjuvanted H5N1 SU vaccine on survival and clinical signs of infection in ferrets challenged with A/Vietnam/1203/04 (H5N1) influenza virus.

\begin{tabular}{|c|c|c|c|c|c|c|}
\hline \multirow{2}{*}{$\begin{array}{l}\text { Group } \\
\text { name }\end{array}$} & \multirow{2}{*}{$\begin{array}{l}\text { Vaccination regimen (days } \\
\text { prior to challenge) }\end{array}$} & \multirow{2}{*}{$\begin{array}{c}\text { No. } \\
\text { dead/ } \\
\text { total no. }\end{array}$} & \multicolumn{4}{|c|}{ Clinical signs $^{\mathrm{b}}$} \\
\hline & & & $\begin{array}{l}\text { Respiratory } \\
\text { symptoms }\end{array}$ & $\begin{array}{l}\text { Neurological } \\
\text { symptoms }\end{array}$ & $\begin{array}{l}\text { Lethargy } \\
\text { (RII) }\end{array}$ & $\begin{array}{c}\text { Weight } \\
\text { loss }\end{array}$ \\
\hline A7 & $\begin{array}{c}15 \mu \mathrm{g} \text { H5N1 Vaccine }+ \text { Alum } \\
(7)\end{array}$ & $0 / 3$ & $2 / 3$ & $0 / 3$ & 1.0 & 11.1 \\
\hline A14 & $\begin{array}{c}15 \mu \mathrm{g} \text { H5N1 Vaccine + Alum } \\
(14)\end{array}$ & $0 / 3$ & $1 / 3$ & $0 / 3$ & 0.0 & -0.4 \\
\hline $\mathbf{A} 21$ & $15 \mu \mathrm{g}$ H5N1 Vaccine + Alum & $0 / 3$ & $0 / 3$ & $0 / 3$ & 0.0 & -1.6 \\
\hline V21 & $15 \mu \mathrm{g} \mathrm{H}$ 5N1 Vaccine (21) & $3 / 3$ & $3 / 3$ & $3 / 3$ & $3 / 3$ & 20.0 \\
\hline
\end{tabular}

${ }^{a}$ Ferrets were immunized with a single $15 \mu \mathrm{g}$ dose of the H5N1 SU vaccine derived from A/Vietnam/1203/04 (H5N1) adjuvanted with Alum. After the indicated number of days after immunization, ferrets were challenged by inoculation with $10^{6} \mathrm{EID}_{50}$ of A/Vietnam/1203/04 (H5N1) virus.

${ }^{\mathrm{b}}$ Clinical signs were observed for 10 days after virus inoculation. Except for lethargy and weight loss, findings for clinical signs are given as no. of ferrets with sign/total no. Respiratory signs included sneezing, wheezing, and nasal discharge; neurological signs included hind limb paresis, ataxia, torticollis, and tremors.

${ }^{\mathrm{c}}$ The relative inactivity index (RII) is scored as follows: 0 , alert and playful; 1 , alert, playful when stimulated; 2 , alert but not playful when stimulated; 3 , neither alert nor playful when stimulated (81).

${ }^{\mathrm{d}}$ Calculated as the mean percentage of weight loss 5 days post challenge (as compared to weight at challenge day). 
Table 2-4. Serological titers and viral load in ferrets immunized with Alumadjuvanted H5N1 SU vaccine and challenged with A/Vietnam/1203/04 (H5N1) influenza virus.

\begin{tabular}{ccccc}
\hline Group $^{\text {a }}$ & $\begin{array}{c}\text { HI } \\
\text { titer }\end{array}$ & $\begin{array}{c}\text { ELISA titer } \\
\text { (IgG) }\end{array}$ & $\begin{array}{c}\text { No. shedding/ total no. of } \\
\text { ferrets tested }^{\mathbf{b}}\end{array}$ & Virus titer $^{\mathbf{c}}$ \\
\hline $\mathbf{A 7}$ & $<$ & $<$ & $1 / 3$ & 3.5 \\
$\mathbf{A 1 4}$ & 23.3 & 900 & $0 / 3$ & NA \\
$\mathbf{A 2 1}$ & 46.6 & 1140 & $0 / 3$ & NA \\
\hline
\end{tabular}

${ }^{\text {a }}$ Vaccination regimen for these groups is shown in Table 2-3.

${ }^{\mathrm{b}}$ Based on the viral titers measured in the nasal washes collected 5 days post challenge with $10^{6} \mathrm{EID}_{50}$ of A/Vietnam/1203/04 virus. The assay detection limit was $0.75 \log _{10}$ $\mathrm{EID}_{50} / \mathrm{mL}$.

${ }^{c}$ Titers (from day 5 nasal washes and expressed as $\log _{10} \mathrm{EID}_{50} / \mathrm{mL}$ ) are given as means \pm $\mathrm{SD}$, unless only 1 animal was shedding virus.

NA, not applicable. 
the scarcity of novel drugs. Several studies (based on mathematical modeling) have suggested that early non-pharmaceutical intervention that would increase social distancing may limit the total number of cases and deaths caused by an emerging pandemic influenza (82-84). However, the efficiency of applying these measures in different communities will be variable. Therefore, prepandemic vaccines remain the cornerstone of pandemic preparedness efforts (77). For more than a decade, avian H5N1 influenza viruses have been a major source of public health concern and continue to pose a pandemic threat (77).Given the high mortality rate associated with human infection with avian $\mathrm{H} 5 \mathrm{~N} 1$ viruses, protection from lethality rather than infection is the paramount goal of various interventions. The preliminary results of this study show, for the first time, that a single adjuvanted $15 \mu \mathrm{g}$ dose of the prepandemic H5N1 subunit vaccine can be protective when administered 7 days prior to lethal H5N1 challenge. Single immunization with the MF59-adjuvanted prepandemic vaccine 14 days prior to challenge resulted in $100 \%$ survival and prevented the development of clinical signs. Shortening the pre-challenge incubation period to 7 days resulted in partial protection (66-100\% survival mild clinical symptoms). Further shortening of the pre-challenge incubation period to 4 days resulted in loss of protection (no survival and severe clinical symptoms). The unadjuvanted prepandemic vaccine failed to provide any protection regardless of the length of the pre-challenge incubation. However, it is important to note that the challenge dose $\left(10^{6} \mathrm{EID}_{50} / \mathrm{ml}\right)$ was highly lethal (around $\left.10^{4} \mathrm{LD}_{50}\right)$. It is reasonable to assume that with a lower dose, we could have started to see protection in ferrets immunized with the unadjuvanted vaccine.

Our preliminary data support what was previously shown by Middleton et al. that a single immunization of adjuvanted prepandemic $\mathrm{H} 5 \mathrm{~N} 1$ vaccine can protect ferrets from challenge-associated mortality in a ferret model $(80)$. In the latter study, however, ferrets were challenged four weeks after immunization; with most of the ferrets had seroconveretd. Our results indicate that the protection from challenge associated death and morbidity observed for M14 ferrets can be correlated with the development of detectable $\mathrm{H} 5$ specific antibody titers in the sera prior to challenge. The anti-H5 titers can be also correlated with decreased load and faster clearance of virus from the upper respiratory tract of M14 ferrets. For H5N1 subunit vaccines, either adjuvanted or unadjuvanted, a pre-challenge incubation period of 4 days was not sufficient to develop an adaptive immune response that can protect against challenge-associated morbidity or mortality.

In our study, it was surprising to see that within the M7 and A7 groups, surviving ferrets did not have any detectable anti-H5 antibody titers either by HI or ELISA. It is important to note that the M7 and A7 ferrets were not completely protected from challenge-associated morbidity. Whereas M14, A14 and A21 ferrets showed minimal clinical symptoms indicating that after a single immunization with an adjuvanted H5N1 vaccine a prechallenge incubation period of 14 days or longer is sufficient for developing protective immune antibody titers. One possible explanation for the early protection against lethality observed in M7 and A7 groups is the development of low affinity $\mathrm{H} 5$-specific IgM antibodies. 
Ferrets are the ideal model for influenza vaccination studies due to the similarities in susceptibility and disease progression between ferrets and humans in the context of influenza infection (26). However, it is important to note that most of the human population, unlike naïve ferrets, is not naïve to influenza virus infection. We were not able to detect any H5 specific antibodies in the sera of ferrets that received the unadjuvanted $\mathrm{H} 5 \mathrm{~N} 1$ vaccine. However, it is important to note that in the case of human immunizations it is possible that cross reactive CD4+ T-cells generated via previous exposure to seasonal influenza viruses in the form of vaccination or infection would play a role in generating anti-H5 immune response to individuals even if they were not previously primed with $\mathrm{H} 5$ vaccines (85). Moreover, cross reacting CD8+ T-cells generated upon infection with seasonal influenza viruses can also play a role in the protection against infection with $\mathrm{H} 5 \mathrm{~N} 1$ viruses $(85)$. We have recently shown that exposure to seasonal influenza viruses immunologically primed for a more robust antibody response to the $2009 \mathrm{H} 1 \mathrm{~N} 1$ monovalent inactivated vaccine (86). The protection was partially mediated by cross reacting CD8+ T-cells (86). This was despite the lack of detectable cross neutralizing antibody responses between the seasonal and the 2009 pandemic H1N1 influenza viruses $(39,86)$.

Since their emergence, H5N1 viruses have evolved into at least 10 phylogenetically and antigenically different clades and multiple subclades (87). While only four of these clades have been associated with human infections, we are unable to predict which clade will acquire the ability to transmit efficiently among humans and thus cause a pandemic (87). In our study, we used a homologous challenge approach; the vaccine antigen and the challenge virus belonged to the same clade. The kinetics of developing protective antibody responses will vary depending on how closely or distantly related the vaccine antigen and the challenge virus in terms of antigenicity. However, in addition to its ability to significantly enhance the immunogenicity of subunit influenza vaccines, MF59 was shown to broaden the antibody responses to seasonal and avian influenza vaccines $(88,89)$. Moreover, MF59 was shown to expand the repertoire of the responding antibodies compared to the unadjuvanted subunit $\mathrm{H} 5 \mathrm{~N} 1$ vaccines (59).

Another important aspect of controlling an emerging influenza pandemic is the ability to limit virus transmission from infected individuals to healthy ones (77). While we have shown enhanced viral clearance in ferrets immunized with the adjuvanted vaccines 14 or 21 days prior to challenge, our results do not exclude the possibility that those ferrets were still able to transmit the virus early after infection. Also it will be important to determine the duration of this protective response and whether a booster immunization will be needed to keep the protective antibody levels.

Milne et al. investigated (using simulation math models) the optimum strategies for utilizing the stockpiled pre-pandemic human H5N1 vaccines (90). However, in this study (and other similar studies) it is assumed that two doses of the vaccine will be required for full protection (90). This assumption was based the observations that individuals do not seroconvert prior to receipt of a second dose (91) and the lack of experimental data regarding the time needed to develop protective immune response following immunization. Based on our results, we think such assumptions will need to be 
reevaluated. From the public health perspective, eliciting an early, within 7 days, protective antibody response with a single immunization has two important implications; it would significantly decrease the amount of antigen required per individual to achieve protection and consequentially expand the vaccine coverage. Secondly, as several reports have indicated, the sole dependence on serological titers as an indicator for protection needs to be reassessed. 


\section{CHAPTER 3. ADJUVANTS' IMPACT ON THE BREADTH OF THE ANTIBODY RESPONSES TO SUBUNIT H5N1 INFLUENZA VACCINES}

\section{Introduction}

Since their emergence in 1996, the goose Guangdong lineage of H5N1 viruses have evolved into at least 10 phylogenetically and antigenically distinct clades (92). Our scientific knowledge falls short of being able to predict which of these clades could adapt to humans and potentially cause a pandemic. Ideally, H5N1 vaccines should stimulate antibody responses that target as many of the shared epitopes present within the 10 clades as possible. Considering the importance of a diversified antibody response to HA within influenza vaccines, it is surprising that we understand little about the factors involved in driving such a response.

Due to their poor immunogenicity, the use of adjuvants with pre-pandemic H5N1 influenza vaccines is strongly recommended (http://www.who.int/csr/resources/publications/WHO HSE EPR GIP 2008 1d.pdf). Bernstein and colleagues have reported that the antibody titers elicited by unadjuvanted or alum-adjuvanted $\mathrm{H} 5 \mathrm{~N} 1$ vaccines are significantly lower than those observed with vaccines that included the oil-in-water adjuvant, MF59 (69). MF59 has been shown, in multiple clinical trials with both seasonal and pandemic vaccines, to enhance antibody titers to homologous influenza strains and to also provide immunity to antigenically distinct virus strains (93-95). In the context of pre-pandemic vaccines, where the potential target virus is unknown, such a broad response is very desirable. Despite these claims, however, it is a little unclear if the cross reactivity noted is due to a qualitative difference in the specificity of the antibody response or if it is simply a reflection of the higher overall titers, i.e., the specificity of an adjuvanted vaccine induced antibody is the same but that minor populations have been elevated to above detectable levels. Malherbe and colleagues have shown that different adjuvants can promote the accumulation of distinct dominant clonotypes of $\mathrm{CD}^{+}{ }^{+} \mathrm{T}$-cells in the context of protein vaccination (96). These authors concluded that, perhaps due to differential activation of dendritic cell populations, different adjuvants can modify the TCR-based selection threshold. As such the pressure for selection of dominant $\mathrm{T}$ cell clones is altered resulting in functionally different response (96). These data suggest that including adjuvants induce qualitative differences in the humoral response to vaccination, either through direct adjuvant action on antigen presentation to B cells or, indirectly, through differential T helper function.

The aim of this chapter was to investigate the diversity of an antibody response to $\mathrm{H} 5 \mathrm{~N} 1$ vaccines generated under different priming environments. Although a number of studies have addressed these issues at the level of antibody quantity, little is known about the quality of these responses. To address this issue, we evaluated the B-cell responses in mice primed with unadjuvanted, alum-adjuvanted, or MF59-adjuvanted H5N1-inactivated vaccine. 


\section{Materials and Methods}

\section{Viruses}

The influenza A/Vietnam/1203/04 (H5N1) virus was obtained from the World Health Organization (WHO) collaborating laboratories. Stock virus was grown in the allantoic cavities of 9-day-old embryonated chicken eggs for $32 \mathrm{~h}$ at $36^{\circ} \mathrm{C}$, and aliquots were stored at $-70^{\circ} \mathrm{C}$ until used. Virus titer was determined by calculating the egg infectious dose $50\left(\mathrm{EID}_{50}\right)$.

\section{Mice and Immunization}

C57BL/6 mice were housed in a pathogen-free facility, and the animal studies were conducted under protocols approved by the St. Jude Children's Research Hospital Committee on Use and Care of Animals. Animals were immunized intramuscularly with the monovalent H5N1 influenza (rgA/Vietnam/1203/2004) subvirion vaccine (Aventis Pasteur Inc., Swiftwater, PA) either unadjuvanted (in PBS), mixed with MF59 (1:1) or adsorbed to aluminium hydroxide at $14.1 \mathrm{mg} / \mathrm{ml}$ Alum, $3 \mu \mathrm{g} / \mathrm{ml}$ antigen and $5 \mathrm{mM}$ Histidine buffer $\mathrm{pH} 6.5$ at $4^{\circ} \mathrm{C}$ overnight. Each mouse dose contained $500 \mu \mathrm{g}$ alum. For all immunizations, the period between the priming and boosting dose was three weeks. The intranasal infections were performed with 100 egg infectious dose 50 of the rg A/Vietnam/1203/2004 diluted in PBS to a final volume of $30 \mu 1$.

\section{Hemagglutination Inhibition Assay}

PBS containing four agglutinating units of A/Vietnam/1203/2004 (H5N1) virus was incubated with serial 2-fold dilutions of RDE-treated serum samples (starting at a 1:10 dilution) at room temperature for 30 minutes. The HI titers of the samples were determined by testing agglutination of $0.5 \%$ of chicken red blood cells.

\section{Elispot Assay}

Ninety-six-well nitrocellulose-bottomed multiscreen HA filtration plates (Millipore, Billerica, MA) were coated $(0.1 \mu \mathrm{g} /$ well $)$ with $\mathrm{H} 5$ protein of the A/Vietnam/1203/2004 (H5N1) virus (e.Enzyme, Montgomery Village, MD). After overnight incubation at $4^{\circ} \mathrm{C}$, the plates were washed with PBS and subsequently blocked. Next, different dilutions of bone marrow cell suspensions were added in volumes of 100 $\mu \mathrm{l} /$ well. After 4 hours of incubation at $37^{\circ} \mathrm{C}$ in a humidified atmosphere containing $5 \%$ $\mathrm{CO}_{2}$, the plates were washed with PBS, and alkaline phosphatase-conjugated goat antimouse IgG (SouthernBiotech, Birmingham, AL) diluted in PBS containing 5\% bovine serum albumin was added $(100 \mu \mathrm{l} /$ well $)$ and incubated overnight at $4^{\circ} \mathrm{C}$. Spots were developed by adding $100 \mu \mathrm{l} /$ well of BCIP/NBT phosphatase substrate (Kirkegaard \& 
Perry Laboratories, Gaithersburg, MD), then counted with an Olympus SZX9 stereozoom microscope for analysis using KS Elispot software (Zeiss, Thornwood, NY).

\section{Enzyme-Linked Immunosorbent Assay (ELISA)}

In brief, microtiter plates (Corning Inc., Corning, NY) were coated overnight at $4^{\circ} \mathrm{C}(0.1 \mu \mathrm{g} /$ well) with $\mathrm{H} 5$ protein of the A/Vietnam/1203/2004 (H5N1) virus (e.Enzyme, Montgomery Village, MD). Plates were then washed with PBS containing $0.05 \%$ Tween 20 and subsequently blocked. Influenza-specific antibody responses were detected with goat anti-mouse IgM, IgG, IgG1, IgG2b, IgG2c, IgG3, or IgE conjugated to alkaline phosphatase (SouthernBiotech, Birmingham, AL). A substrate (p-nitrophenyl phosphate; Sigma-Aldrich, St. Louis, MO) was added, plates were incubated for 30 minutes at room temperature for color development, and optical density values were determined at $405 \mathrm{~nm}$ in an ELISA reader (Molecular Devices, Sunnyvale, CA). Titers were presented as the highest dilution that yielded an optical density three times greater than that of a $1 / 100$ dilution of pre-immune serum. Serum IgG levels were determined using a mouse IgG ELISA kit (Life Diagnostics, West Chester, PA).

\section{Competitive ELISA}

The H5 specific human mAbs were purified on protein A or G columns (GE Healthcare, Smyrna, GA). The competition between murine polyclonal serum antibodies and human $\mathrm{mAbs}$ for binding to immobilized antigens was measured by ELISA. Briefly, the $\mathrm{mAbs}$ were added to HA-coated plates at a high concentration $(10 \mathrm{mg} / \mathrm{ml})$. After 1 hour, plasma samples were added at a concentration corresponding to $70 \%-80 \%$ of the maximal OD level, and the mixture was incubated at room temperature for 1 hour. Plates were then washed and bound sera were detected using AP-labeled anti-mouse IgG antibodies (Southern biotech, Birmingham, AL). The percentage of inhibition was tested in duplicates and calculated as follows: $\left[\left(\mathrm{OD}^{\text {no-competition }}-\mathrm{OD}^{\text {competition }}\right) /\left(\mathrm{OD}^{\text {no-competition }}\right)\right]$ $\times 100$.

\section{Flow Cytometry}

Mice were euthanized via $\mathrm{CO}_{2}$ inhalation and inguinal lymph nodes were harvested after immunization. Lymph nodes were manually disrupted by grinding organ tissue between the frosted ends of two sterile glass microscope slides in sterile PBS containing $2 \%$ fetal bovine serum. Cells were then labeled with anti-CD16/CD32 (clone 2.4G2, BD PharMingen, San Diego, CA) to block nonspecific Fc receptor-mediated binding. After washing, cells were stained with different combinations of fluorescently labelled antibodies against CD8 $\alpha$ (clone 53-6.7, BD PharMingen), CD4 (clone RM4-5, BD PharMingen), CD11b (M1/70, Biolegend, San Diego, CA), IgD (11-26c.2a, BD PharMingen), CD138 (281-2, BD PharMingen), B220 (RA3-6B2, BD PharMingen), FAS (Jo2, BD PharMingen), and GL7 (BD PharMingen). Labeled cells were assayed with a 
FACSCalibur flow cytometer (BD Biosciences, Franklin Lakes, NJ) and data were analyzed using FlowJo (Tree Star, Ashland, OR) software.

\section{PCR Amplifications and High-Throughput Pyrosequencing and Analysis of the Immunoglobulin Heavy Chain Variable Genes}

PCR amplifications were performed using total RNA as template for each reaction. Following PCR, amplicons were then purified by $1.5 \%$ agarose gel electrophoresis and gel extraction as described previously. PCR was performed using primers that were previously described (97) but were modified to fit the 454 technology. High-throughput amplicon pyrosequencing data obtained with a 454 instrument performed using the Titanium chemistry, with amplicons sequencing reads beginning from the "A" primer in the manufacturer's protocol in both cases (Roche, Branford, CT). Additional 12-nt sample bar codes were incorporated into the multiplexed IGHV gene segment primers. Sequence bar codes allowed for the pooling and bulk sequencing of many libraries and the subsequent retrieval of sequences that were derived from each sample. The sequences were analyzed using the data base ImMunoGeneTics (http://imgt.cines.fr/) and the number of non-silent mutations within the variable genes was compared. Clonal analysis was performed on these sequences based on the assumption that if two or more sequences shared the same variable heavy chain, diversity and junction genes usage and the same junction sequence, these sequences are considered a clonal pool. The number of non-silent mutations was determined for each clonal pool.

\section{H5 Antigen Microarray}

Antigen arrays are a high-throughput assay that builds upon the technology of DNA microarrays. Slides coated with an epoxy surface were spotted using a robotic microarray printer. The slides were printed with 54 of overlapping 18mers spanning the full length of H5 HA from A/Vietnam/1203/2004 (H5N1) (Table 3-1). Slides were then blocked in BSA $1 \%$ and then incubated with purified IgG (Protein G, GE Healthcare, Smyrna, GA) from the sera derived from naïve and immunized C57bl/6 mice. After washing, the slides were incubated with a 2 nd anti-IgG that are fluorescent tagged. Slides are washed, dried and then scanned in a laser scanner.

\section{Statistical Analysis}

Statistical analyses were performed using InStat (GraphPad, La Jolla, CA) software. In most cases, the Mann-Whitney (non-parametric) test was used to compare results. 
Table 3-1. List of peptide sequences that are used to coat H5 microarray chips.

\begin{tabular}{|c|c|c|c|}
\hline No. & Sequence & No. & Sequence \\
\hline 1 & K-I-V-L-L-F-A-I-V-S-L-V-K-S-D-Q-I-C & 28 & V-K-K-G-D-S-T-I-M-K-S-E-L-E-Y-G-N-C \\
\hline 2 & L-V-K-S-D-Q-I-C-I-G-Y-H-A-N-N-S-T-E & 29 & S-E-L-E-Y-G-N-C-N-T-K-C-Q-T-P-M-G-A \\
\hline 3 & Y-H-A-N-N-S-T-E-Q-V-D-T-I-M-E-K-N-V & 30 & K-C-Q-T-P-M-G-A-I-N-S-S-M-P-F-H-N-I \\
\hline 4 & D-T-I-M-E-K-N-V-T-V-T-H-A-Q-D-I-L-E & 31 & S-S-M-P-F-H-N-I-H-P-L-T-I-G-E-C-P-K \\
\hline 5 & T-H-A-Q-D-I-L-E-K-K-H-N-G-K-L-C-D-L & 32 & L-T-I-G-E-C-P-K-Y-V-K-S-N-R-L-V-L-A \\
\hline 6 & H-N-G-K-L-C-D-L-D-G-V-K-P-L-I-L-R-D & 33 & K-S-N-R-L-V-L-A-T-G-L-R-N-S-P-Q-R-E \\
\hline 7 & V-K-P-L-I-L-R-D-C-S-V-A-G-W-L-L-G-N & 34 & L-R-N-S-P-Q-R-E-R-R-R-K-K-R-G-L-F-G \\
\hline 8 & V-A-G-W-L-L-G-N-P-M-C-D-E-F-I-N-V-P & 35 & R-K-K-R-G-L-F-G-A-I-A-G-F-I-E-G-G-W \\
\hline 9 & C-D-E-F-I-N-V-P-E-W-S-Y-I-V-E-K-A-N & 36 & A-G-F-I-E-G-G-W-Q-G-M-V-D-G-W-Y-G-Y \\
\hline 10 & S-Y-I-V-E-K-A-N-P-V-N-D-L-C-Y-P-G-D & 37 & M-V-D-G-W-Y-G-Y-H-H-S-N-E-Q-G-S-G-Y \\
\hline 11 & N-D-L-C-Y-P-G-D-F-N-D-Y-E-E-L-K-H-L & 38 & S-N-E-Q-G-S-G-Y-A-A-D-K-E-S-T-Q-K-A \\
\hline 12 & D-Y-E-E-L-K-H-L-L-S-R-I-N-H-F-E-K-I & 39 & D-K-E-S-T-Q-K-A-I-D-G-V-T-N-K-V-N-S \\
\hline 13 & R-I-N-H-F-E-K-I-Q-I-I-P-K-S-S-W-S-S & 40 & G-V-T-N-K-V-N-S-I-I-D-K-M-N-T-Q-F-E \\
\hline 14 & I-P-K-S-S-W-S-S-H-E-A-S-L-G-V-S-S-A & 41 & D-K-M-N-T-Q-F-E-A-V-G-R-E-F-N-N-L-E \\
\hline 15 & A-S-L-G-V-S-S-A-C-P-Y-Q-G-K-S-S-F-F & 42 & G-R-E-F-N-N-L-E-R-R-I-E-N-L-N-K-K-M \\
\hline 16 & Y-Q-G-K-S-S-F-F-R-N-V-V-W-L-I-K-K-N & 43 & I-E-N-L-N-K-K-M-E-D-G-F-L-D-V-W-T-Y \\
\hline 17 & V-V-W-L-I-K-K-N-S-T-Y-P-T-I-K-R-S-Y & 44 & G-F-L-D-V-W-T-Y-N-A-E-L-L-V-L-M-E-N \\
\hline 18 & Y-P-T-I-K-R-S-Y-N-N-T-N-Q-E-D-L-L-V & 45 & E-L-L-V-L-M-E-N-E-R-T-L-D-F-H-D-S-N \\
\hline 19 & T-N-Q-E-D-L-L-V-L-W-G-I-H-H-P-N-D-A & 46 & T-L-D-F-H-D-S-N-V-K-N-L-Y-D-K-V-R-L \\
\hline 20 & G-I-H-H-P-N-D-A-A-E-Q-T-K-L-Y-Q-N-P & 47 & N-L-Y-D-K-V-R-L-Q-L-R-D-N-A-K-E-L-G \\
\hline 21 & Q-T-K-L-Y-Q-N-P-T-T-Y-I-S-V-G-T-S-T & 48 & R-D-N-A-K-E-L-G-N-G-C-F-E-F-Y-H-K-C \\
\hline 22 & Y-I-S-V-G-T-S-T-L-N-Q-R-L-V-P-R-I-A & 49 & C-F-E-F-Y-H-K-C-D-N-E-C-M-E-S-V-R-N \\
\hline 23 & Q-R-L-V-P-R-I-A-T-R-S-K-V-N-G-Q-S-G & 50 & E-C-M-E-S-V-R-N-G-T-Y-D-Y-P-Q-Y-S-E \\
\hline 24 & S-K-V-N-G-Q-S-G-R-M-E-F-F-W-T-I-L-K & 51 & Y-D-Y-P-Q-Y-S-E-E-A-R-L-K-R-E-E-I-S \\
\hline 25 & E-F-F-W-T-I-L-K-P-N-D-A-I-N-F-E-S-N & 52 & R-L-K-R-E-E-I-S-G-V-K-L-E-S-I-G-I-Y \\
\hline 26 & D-A-I-N-F-E-S-N-G-N-F-I-A-P-E-Y-A-Y & 53 & K-L-E-S-I-G-I-Y-Q-I-L-S-I-Y-S-T-V-A \\
\hline 27 & F-I-A-P-E-Y-A-Y-K-I-V-K-K-G-D-S-T-I & 54 & L-S-I-Y-S-T-V-A-S-S-L-A-L-A-I-M-V-A \\
\hline
\end{tabular}




\section{Results}

\section{MF59 is Superior to Alum in Priming for Robust H5-Specific Antibody Responses}

HA-specific antibodies are considered the most important correlate of protection elicited by inactivated influenza vaccines. It was previously shown that in mice MF59 is superior to alum and several other adjuvants in priming for a robust antigen-specific humoral immune response when combined with seasonal influenza vaccines (98). To test this observation in the context of pre-pandemic H5N1 vaccines, we primed four groups of WT C57BL/6 mice with H5N1 subvirion vaccines (MF59-, alum-adjuvanted or unadjuvanted) or with sub lethal $\mathrm{H} 5 \mathrm{~N} 1$ influenza virus (homologous to the vaccine strain) infection. After boosting all groups with the unadjuvanted H5N1 subvirion vaccine in phosphate buffered saline (PBS), we first determine the serum levels of H5-specific IgG antibodies induced. We found that mice primed with the MF59 adjuvanted vaccine elicited significantly higher antibody titers than the alum-adjuvanted and unadjuvanted vaccine-primed mice, though they were comparable to those elicited in the infection-primed mice (Fig. 3-1A). Mean pre-boost titers for the MF59, alum and unadjuvanted vaccine groups were $3400,362.5$ and 25 respectively. We next determined the frequency of H5-specific antibody forming cells (AFCs) in the bone marrow and spleen after the boosting immunization. For the unadjuvanted vaccine-primed mice, the number of AFCs was below the assay detection limit (Fig. 3-1B-C). However, and consistent with the serum IgG results, the number of H5-specific IgG forming cells was comparably elevated in the MF59- and infection-primed mice and was significantly higher than that observed in alum-primed mice (Fig. 3-1B-C).

\section{MF59 and Alum Enhance the Affinity Maturation of the B-Cell Response to H5N1 Vaccine}

One of the important aspects of adjuvants' impact on the antibody responses to immunization is their ability to enhance the affinity of vaccine specific antibodies (99). Affinity maturation is believed to occur in the B-cell follicles during the germinal center reaction where B-cell receptors are subjected to multiple rounds of mutations known as somatic hypermutation (100). Emerging B-cell clones with the highest affinity gain a survival advantage and they form a pool of memory B-cells and long lived plasma cells (100). This process requires CD4+ T-cell help. These mutations are largely clustered in the complementarity determining regions of the antibody variable genes (101). We investigated the impact of MF59 and alum on the maturity of B-cell responses to H5N1 vaccines. Spleenocytes from mice immunized as described in Fig. 3-1 were harvested 5 days following the final boost and the total number of B-cells differentiated into germinal center B-cells (GCs) in the different groups was determined. Consistent with the antibody titers, priming with the MF59-adjuvanted H5N1 vaccine led to a greater accumulation of B-cells within GCs compared to the unadjuvanted or the alum-adjuvanted vaccine (Fig. 3-2A). The MF59 induced GC accumulation was equivalent to that observed in mice primed with sublethal H5N1 infection (Fig. 3-2A). 

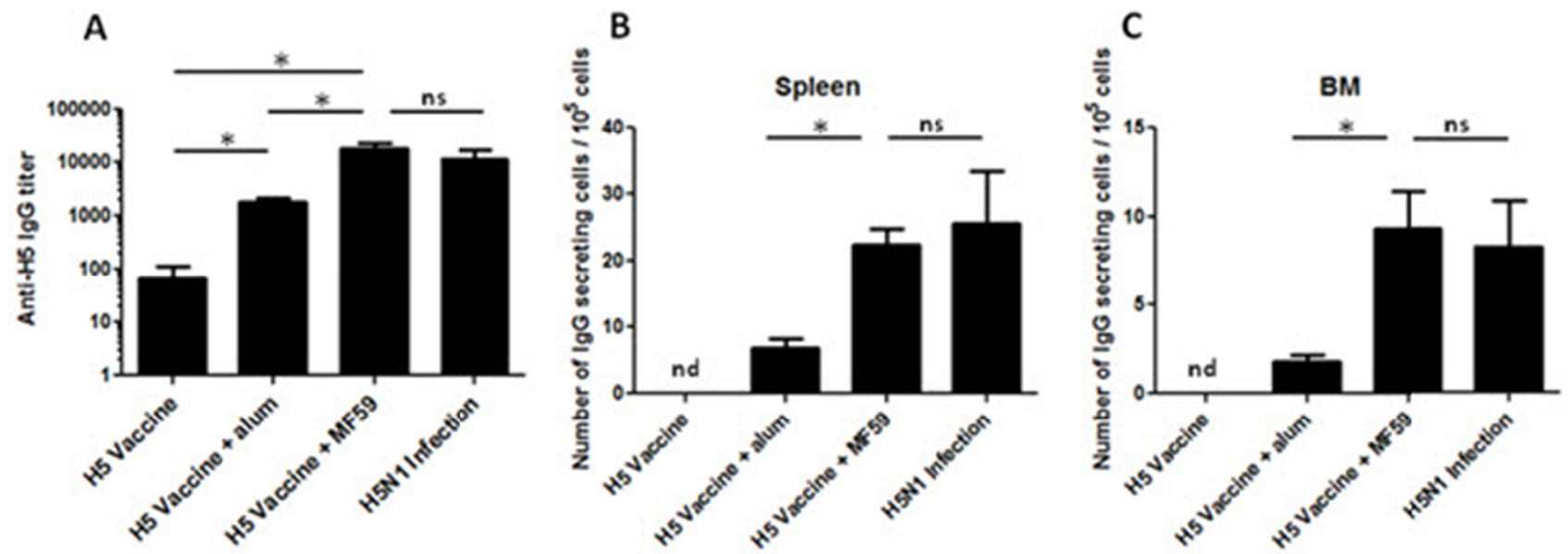

Figure 3-1. Superior priming with MF59-adjuvanted H5N1 vaccines in comparison to the alum-adjuvant or the unadjuvanted formulations.

Four groups of C57BL/6 wild-type mice ( $\mathrm{n}=5$ ) were intramuscularly primed either with $1 \mu \mathrm{g}$ of H5N1 subvirion vaccine (MF59-, alum-adjuvanted or unadjuvanted) or with sub lethal A/vietnam/1203/2004 (H5N1) influenza virus infection. All groups were boosted with $3 \mu \mathrm{g}$ of $\mathrm{H} 5 \mathrm{~N} 1$ subvirion vaccine in PBS and five days after the boost sera and other organs were harvested. $(A)$ Mean circulating levels of H5-specific IgG antibodies. (B) Mean number of H5-specific IgG forming cells in the bone marrow and $(C)$ spleen. The results are representative of at least 2 separate experiments. Statistically significant differences between WT and mutant groups are as indicated with asterisks; ${ }^{*}, \mathrm{p}<0.05$. ns, not significant. nd, not detectable. Error bars show standard error. 

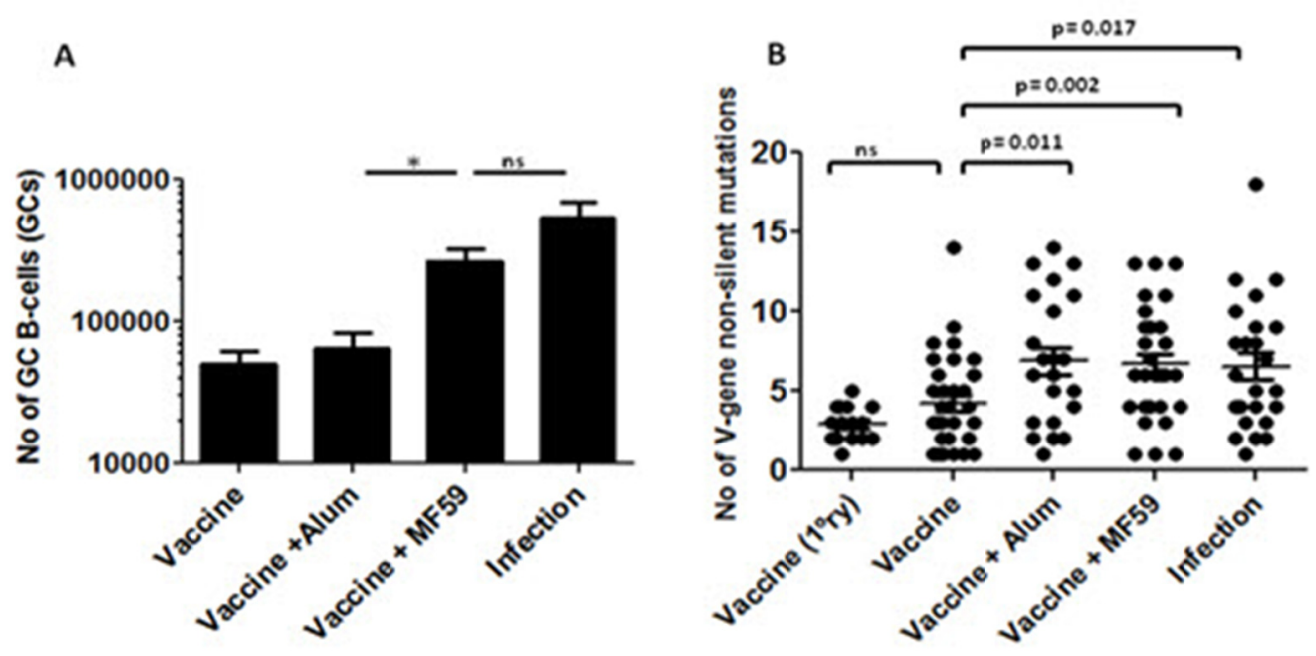

Figure 3-2. Increased affinity maturation of the B-cell responses mediated by alumand MF59-adjuvanted H5N1 vaccines.

Four groups of C57BL/6 wild-type mice $(n=5)$ were intramuscularly primed either with 1 $\mu \mathrm{g}$ of H5N1 subvirion vaccine (MF59-, alum-adjuvanted or unadjuvanted) or with sub lethal A/Vietnam/1203/2004 (H5N1) influenza virus infection. All groups were boosted with $3 \mu \mathrm{g}$ of H5N1 subvirion vaccine in PBS. (A) Shown are absolute numbers of the germinal center B-cells in spleens five days after the last booster immunization. $(B)$ Mean number of non-silent mutations within the IGHV genes. ns, not significant. Error bars show standard error. 
To determine the extent of affinity maturation in the responding B-cells, we sorted antibody forming cells (AFCs) from the draining (inguinal) lymph nodes by flow cytometry $\left(\mathrm{CD} 4^{\text {neg }}, \mathrm{CD} 8^{\text {neg }}, \mathrm{IgD}^{\text {neg }}, \mathrm{B} 220^{\text {low }}\right.$ and $\mathrm{CD} 138^{\text {pos }}$ cells) and total RNA was isolated. More than $80 \%$ of the IgG producing cells were specific to H5 by Elispot assay (not shown). Next, the cDNA was used as a template to amplify the immunoglobulin heavy chain variable (IGHV) genes. The PCR amplicons were sequenced by using the 454 deep sequencing (see Materials and Methods). Additionally, a group of mice that were immunized once with the unadjuvanted vaccine was included. We observed that similar to the infection group, including alum and MF59 adjuvants in the priming immunization enhanced affinity maturation of the recalled B-cell response compared to priming with the unadjuvanted vaccine (Fig. 3-2B).

\section{Similar Binding Pattern by Adjuvanted and Unadjuvanted H5 Immune Serum-Derived IgGs to H5 HA Antigen Microarray}

After establishing that adjuvanted $\mathrm{H} 5 \mathrm{~N} 1$ vaccines elicited quantitatively greater H5-specific antibody titers than the unadjuvanted vaccine preparation, we wanted to further explore differences in quality by considering the range of target specificity induced by each priming regimen. We tested whether there are differences in the specificity of binding to linear epitopes of the H5 HA protein. To this end we constructed $\mathrm{H} 5$ antigen microarrays using 54 peptides that span the full length of H5 HA of A/Vietnam/1203/2004 (H5N1). The peptides are 18mers with 10 amino acids overlap and were spotted on epoxy glass slides as described in the materials and methods section. IgG (normalized to equal IgG content) immunoglobulins purified from sera derived from mice immunized with H5N1 vaccine either adjuvanted (with alum or MF59) or unadjuvanted that were normalized and their binding to the H5 antigen chip was tested. There was minimal binding shown by IgGs purified from sera derived from naïve mice (Fig. 3-3). The pattern of binding of IgGs from the different groups, indicated by the identity of peptides targeted, was similar (Fig. 3-3). These peptides were, however, targeted to different extents among the 4 groups (Fig. 3-3). These data indicate that, at least for $\mathrm{H} 5$ linear epitopes, the composition of the responses elicited by the adjuvanted and unadjuvanted vaccines to such epitopes is similar, but with apparent variation in epitope immundominance.

\section{Similar Inhibition Pattern by Adjuvanted and Unadjuvanted H5 Immune Sera by H5 Monoclonal Antibodies}

A major limitation of the H5 antigen microarray is that it only examines the binding to linear epitopes. It is assumed that many of the neutralizing antibody epitopes on HA are conformational in nature. Therefore, we next examined the binding of the sera from the different groups to epitopes in the globular head of HA. We used a competitive ELISA approach with human monoclonal antibodies (mAbs) that recognize the globular

head and measured their capacity to inhibit the binding of these sera. The binding of the 2 mAbs, 10C3 and 7H5, was mapped to amino acid residues within the globular head of 

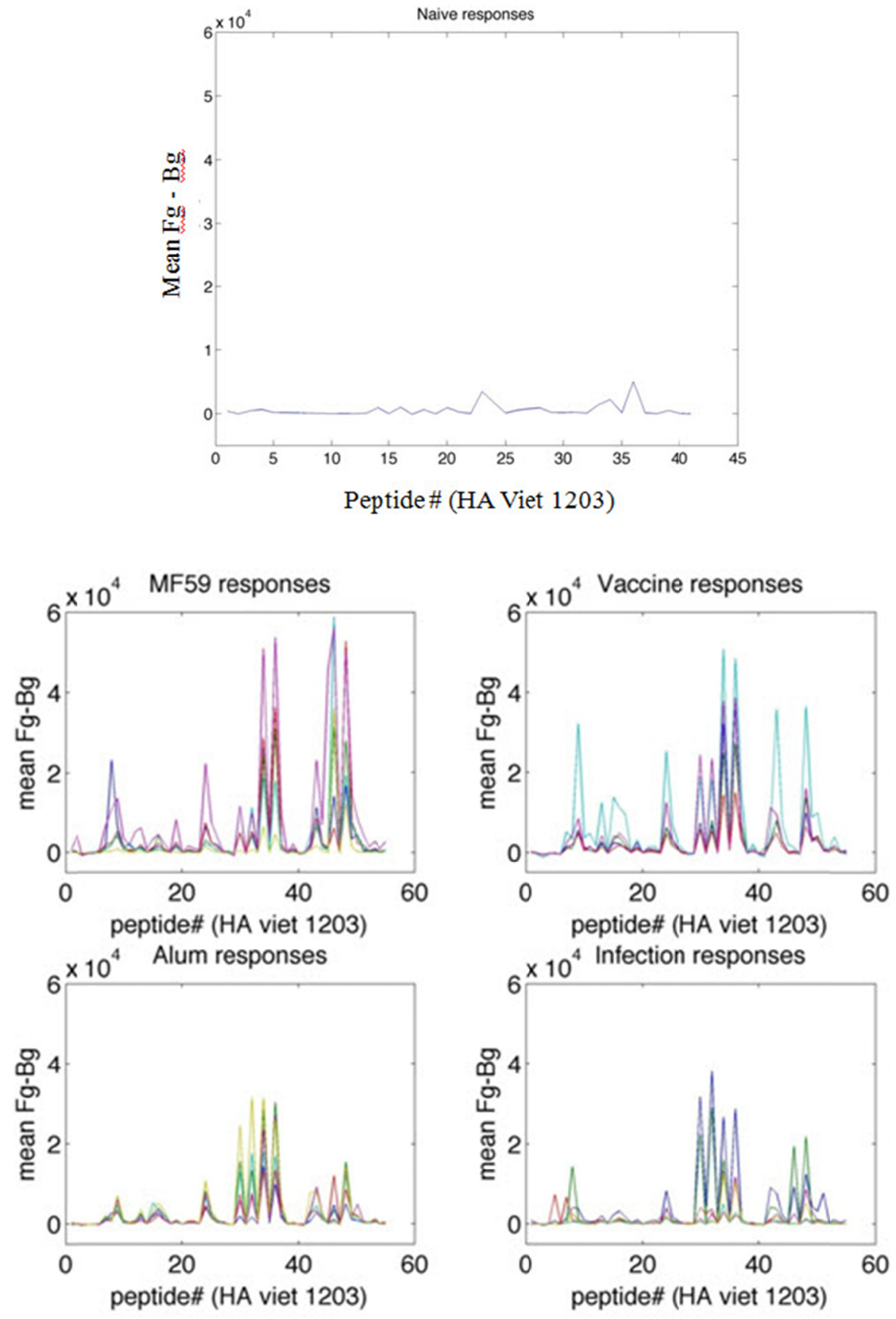

Figure 3-3. Similar pattern of binding of immune sera to H5 antigen microarrays.

IgG immunoglobulins purified from naive mice sera (upper panel) or from mice immunized as described in Fig. 3-1 (lower panel). Normalized IgG content from the different mice groups binding to the H5 antigen microarray as described in the materials and methods section. The different coloured lines within each group represent different mice $(n=6)$. 
the H5 HA. 10C3 binding was mapped to the amino acid residue 145 which lies in antigenic site 1 of H5 HA (29) and 7H5 binds to the amino acid residues 154 and156 which represent antigenic site 2 of H5 HA (29). ELISA plates were coated with recombinant H5 HA (A/Vietnam/1203/04) and incubated with or without an excess of the human mAbs followed by addition of mice sera at a dilution that gives $70-80 \%$ of maximum binding to all plates that were then detected using mouse-specific secondary reagents. We found that binding of the sera from the four groups was inhibited to similar extent by the two mAbs (Fig. 3-4A-B). This indicates that the proportions of antibodies targeting the epitopes blocked by these $2 \mathrm{mAbs}$ among the different sera groups are equivalent (Fig. 3-4A-B) and that the response to these epitopes is a substantial population of the overall response.

\section{Discussion}

While the use of vaccines to protect humans against infectious diseases has been introduced over two centuries ago, only a few adjuvants are currently licensed for use in humans. In the context of influenza vaccines, this is partially because vaccines were traditionally formulated as whole inactivated viruses, which are strongly immunogenic. Currently, inactivated influenza vaccines are mostly formulated as split or subunit vaccines, which mainly contain purified HA and NA. While these subunit preparations have induced minimal adverse reactions, they displayed poor immunogenicity in clinical trials (78). The use of adjuvants with subunit vaccines has significantly boosted the immunogenicity of such vaccines (69). While the quantitative outcome of including adjuvants with influenza vaccines is well documented, the impact of these adjuvants on the quality of immune response to influenza vaccines is still unclear. Therefore, it is becoming increasingly important to determine how clinically relevant adjuvants such as alum and MF59 modulate the immune responses to influenza vaccines. More specifically, we examined whether or not including adjuvants induces a broader response to influenza vaccines compared to unadjuvanted preparations. Our data clearly show that MF59-adjuvanted H5N1 vaccines elicits a quantitatively greater H5-specific antibody response than the alum-adjuvanted or the unadjuvanted vaccine. These titers were consistent with the ability of MF59-adjuvanted vaccine to prime for more robust H5-specific B-cell responses numerically, generating greater numbers of germinal center $B$ cells. However, for the most part, the specificity of these antibodies was not different than induced by alum or vaccine alone as determined by and $\mathrm{H} 5$ antigen microarray. Additionally, competitive inhibition studies using antibodies that target prominent epitopes on the HA globular head indicated that these epitopes were targeted to a similar extent. It is important to note that although the alum-adjuvanted vaccine responses were inferior to those induced by MF59, the activated B-cells showed an equivalent degree of maturation. Our results may appear to contrast previous reports, which suggested that including MF59 with H5N1 and seasonal influenza vaccines enhance antibody titers to drifted (from the vaccine parent strain) virus strains (88, 93-95). Moreover, Khurana and colleagues have tested the binding pattern of sera obtained from $\mathrm{H} 5 \mathrm{~N} 1$ vaccine clinical trials using influenza-specific whole-genome fragment phage display libraries and concluded that the use of MF59 expanded the number of epitopes targeted when 
A

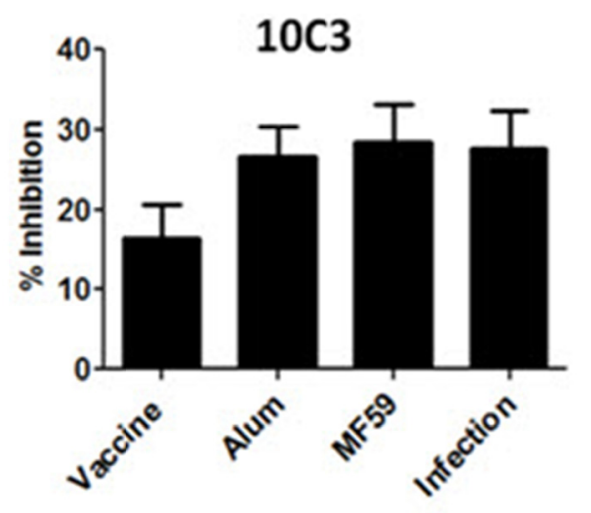

B

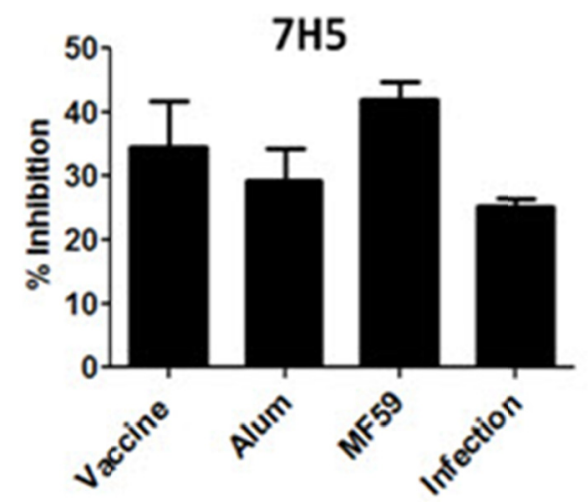

Figure 3-4. Equivalent inhibition of adjuvanted and unadjuvanted H5 immune sera by H5 human monoclonal antibodies.

Sera were taken from C57BL/6 mice immunized as described in Fig. 3-1. Competitive binding to full length HA was performed as described in methods in presence of $\mathrm{H} 5$-spcific the monoclonal antibodies 10C3 $(A)$ and 7H5 $(B)$. Error bars show standard error. 
combined with $\mathrm{H} 5 \mathrm{~N} 1$ vaccines (59). The explanation for these discrepancies may be explained in light of two factors: 1) the assay used to estimate cross-reactivity is the hemagglutination inhibition (HI) assay. $\mathrm{HI}$ is the standard assay used in evaluating the efficacy of influenza vaccines and it detects antibodies that block receptor binding. The sensitivity of the assay is low, which in turn may result in overlooking antibody responses to sub-dominant epitopes in the case of unadjuvanted vaccine responses. With the MF59-adjuvanted responses, however, the immune response is greatly enhanced to antibody levels targeting these sub-dominant epitopes exceeding the assay detection limit. Overall though, the target specificities are shared. 2) MF59-adjuvanted vaccines activate antigen-specific memory $\mathrm{CD} 4^{+} \mathrm{T}$-cells to a greater extent compared to the unadjuvanted preparations (102). This activation might have led to a greater involvement of pre-existing memory B-cell populations upon immunization. In support of this notion is that in contrast to our studies with naïve mice, the data from which the adjuvant induced "breadth" of antibody responses assumption was derived come mainly from clinical trials. Therefore, the observed specificity differences between the adjuvanted and unadjuvanted responses may stem from that humans have pre-existing specificities that an unadjuvanted vaccination may not be able to generate. 


\section{CHAPTER 4. INFLAMMASOME-INDEPENDENT ROLE OF THE APOPTOSIS-ASSOCIATED SPECK-LIKE PROTEIN CONTAINING CARD (ASC) IN THE ADJUVANT EFFECT OF MF59²}

\section{Introduction}

The primary means of infection- and vaccine-mediated immunologic protection against influenza A viruses (IAVs) is the development of antibodies that bind and neutralize the receptor binding function of hemagglutinin (HA). Vaccination is the most efficient means to minimize influenza-mediated illness. However, the results from the first clinical trials of unadjuvanted subunit $\mathrm{H} 5 \mathrm{~N} 1$ vaccines were disappointing in terms of immunogenicity (78). Substantial improvements have, however, been seen in trials with the adjuvanted formulations specifically using the new generation oil-in-water emulsion-based adjuvants that have induced significantly higher antibody responses to low antigen-content vaccines (69).

The main licensed adjuvants used with influenza vaccines such as alum and MF59 have been empirically developed. The molecular mechanism of these two adjuvants is still largely undefined. It has been shown that alum and MF59 induce the secretion of a range of cytokines associated with recruitment of innate immune cells to the injection site $(99,103,104)$. The immunostimulatory activity of alum has been, at least in part, attributed to the ability of alum to activate an innate immune sensor that belongs to the NOD-like receptors (NLRs) family (64). NLRs are a large family of intracellular proteins that are believed to be primarily involved in the innate immune response to microbial pathogens through the recognition of conserved pathogen-associated molecular pattern (105). However, they also contribute by sensing "danger signals," i.e., endogenous molecules that are produced during tissue damage or inflammation (105). Specifically, in NLR-Pyrin domain containing 3- (NLRP3) deficient mice, decreased IL-1 $\beta$ secretion and antigen-specific humoral immune responses to immunization with alum-adsorbed antigens have been observed $(106,107)$.

NLRP3 allows for the recruitment and autocatalytic activation of the cysteine protease caspase-1 in a large cytosolic protein complex named the "inflammasome" (108). Once activated, the inflammasome mediates caspase-1 cleavage of the inactive precursor of the proinflammatory cytokine, IL-1 $\beta$, resulting in release of mature IL-1 $\beta$. The adapter protein ASC bridges the interaction between NLRP3 and the caspase-1, making it essential for activation of the inflammasome (108). Whether or not any of the NLRP3 inflammasome components (NLRP3, ASC, and caspase-1) play a role in MF59-mediated immune stimulation has not been addressed. The goal of this study was to understand the role of the different NLRP3 inflammasome components (NLRP3, ASC, and caspase-1) in MF59-mediated immune stimulation.

${ }^{2}$ This chapter was adapted with permission. A. H. Ellebedy et al., Proc Natl Acad Sci U S A 108, 2927 (Feb 15, 2011) (120). 


\section{Materials and Methods}

\section{Mice and Immunization}

Cryopyrin/Nlrp3 $3^{-/-}, \mathrm{ASC}^{-/-}$, and caspase-1 ${ }^{-/-}$mice were backcrossed to a C57BL/6 background for at least 10 generations. Mice were housed in a pathogen-free facility, and the animal studies were conducted under protocols approved by the St. Jude Children's Research Hospital Committee on Use and Care of Animals. Animals were immunized intramuscularly twice with 1,3 , or $5 \mu \mathrm{g}$ of monovalent H5N1 influenza (rgA/Vietnam/1203/2004) subvirion vaccine (Aventis Pasteur Inc., Swiftwater, PA) either unadjuvanted (in PBS), mixed with MF59 (1:1) or adsorbed to aluminium hydroxide at $14.1 \mathrm{mg} / \mathrm{ml}$ Alum, $3 \mu \mathrm{g} / \mathrm{ml}$ antigen and $5 \mathrm{mM}$ Histidine buffer $\mathrm{pH} 6.5$ at $4^{\circ} \mathrm{C}$ overnight. Incomplete Freund's adjuvant or IFA (Sigma, St. Louis, MO) was mixed with the vaccine solution in 1:1 (v/v) ratio and emulsified just before injection. Each mouse dose contained $500 \mu \mathrm{g}$ Alum. The intranasal infections were performed with $100 \mathrm{egg}$ infectious dose 50 of the $\mathrm{rgA} /$ Vietnam/1203/2004 ( $\triangle \mathrm{HA}$ ) diluted in PBS to a final volume of $30 \mu 1$.

\section{Hemagglutination Inhibition Assay}

PBS containing four agglutinating units of A/Vietnam/1203/2004 (H5N1) virus was incubated with serial 2-fold dilutions of RDE-treated serum samples (starting at a 1:10 dilution) at room temperature for 30 minutes. The HI titers of the samples were determined by testing agglutination of $0.5 \%$ of chicken red blood cells.

\section{Elispot Assay}

Ninety-six-well nitrocellulose-bottomed multiscreen HA filtration plates (Millipore, Billerica, MA) were coated $(0.1 \mu \mathrm{g} / \mathrm{well})$ with $\mathrm{H} 5$ protein of the A/Vietnam/1203/2004 (H5N1) virus (e.Enzyme, Montgomery Village, MD). After overnight incubation at $4^{\circ} \mathrm{C}$, the plates were washed with PBS and subsequently blocked. Next, different dilutions of bone marrow cell suspensions were added in volumes of 100 $\mu \mathrm{l} /$ well. After 4 hours of incubation at $37^{\circ} \mathrm{C}$ in a humidified atmosphere containing $5 \%$ $\mathrm{CO}_{2}$, the plates were washed with PBS, and alkaline phosphatase-conjugated goat anti-mouse IgG (SouthernBiotech, Birmingham, AL) diluted in PBS containing 5\% bovine serum albumin was added $(100 \mu \mathrm{l} /$ well $)$ and incubated overnight at $4^{\circ} \mathrm{C}$. Spots were developed by adding $100 \mu \mathrm{l} /$ well of BCIP/NBT phosphatase substrate (Kirkegaard \& Perry Laboratories, Gaithersburg, MD), then counted with an Olympus SZX9 stereozoom microscope for analysis using KS Elispot software (Zeiss, Thornwood, NY). 


\section{Enzyme-Linked Immunosorbent Assay (ELISA)}

In brief, microtiter plates (Corning Inc., Corning, NY) were coated overnight at $4^{\circ} \mathrm{C}(0.1 \mu \mathrm{g} /$ well) with $\mathrm{H} 5$ protein of the A/Vietnam/1203/2004 (H5N1) virus (e.Enzyme, Montgomery Village, MD). Plates were then washed with PBS containing $0.05 \%$ Tween 20 and subsequently blocked. Influenza-specific antibody responses were detected with goat anti-mouse $\operatorname{IgM}, \operatorname{IgG}, \operatorname{IgG1}, \operatorname{IgG} 2 \mathrm{~b}, \operatorname{IgG} 2 \mathrm{c}, \operatorname{IgG} 3$, or $\operatorname{IgE}$ conjugated to alkaline phosphatase (SouthernBiotech, Birmingham, AL). A substrate (p-nitrophenyl phosphate; Sigma-Aldrich, St. Louis, MO) was added, plates were incubated for 30 minutes at room temperature for color development, and optical density values were determined at $405 \mathrm{~nm}$ in an ELISA reader (Molecular Devices, Sunnyvale, CA). Titers were presented as the highest dilution that yielded an optical density three times greater than that of a 1/100 dilution of pre-immune serum. Serum IgG levels were determined using a mouse $\mathrm{IgG}$ ELISA kit (Life Diagnostics, West Chester, PA).

\section{Flow Cytometry}

Mice were euthanized via $\mathrm{CO}_{2}$ inhalation and inguinal lymph nodes were harvested after immunization. Lymph nodes were manually disrupted by grinding organ tissue between the frosted ends of two sterile glass microscope slides in sterile PBS containing $2 \%$ fetal bovine serum. Cells were then labeled with anti-CD16/CD32 (clone 2.4G2, BD PharMingen, San Diego, CA) to block nonspecific Fc receptor-mediated binding. After washing, cells were stained with different combinations of fluorescently labelled antibodies against CD8 $\alpha$ (clone 53-6.7, BD PharMingen), CD4 (clone RM4-5, BD PharMingen), CD11b (M1/70, Biolegend, San Diego, CA), IgD (11-26c.2a, BD PharMingen), CD138 (281-2, BD PharMingen), B220 (RA3-6B2, BD PharMingen), FAS (Jo2, BD PharMingen), and GL7 (BD PharMingen). Labeled cells were assayed with a FACSCalibur flow cytometer (BD Biosciences, Franklin Lakes, NJ) and data were analyzed using FlowJo (Tree Star, Ashland, OR) software.

\section{Generation and Analysis of Bone Marrow-Derived Dendritic Cells}

BMDC cultures were prepared as described previously (109) and stimulated with MF59 $(1: 100 \mathrm{~V} / \mathrm{V})$ or as indicated in the figure legends for 24 hours. Cell culture supernatants were harvested, and the different cytokines were assayed using Milliplex Map kits (Millipore, Billerica, MA).

\section{Statistical Analysis}

Statistical analyses were performed using InStat (GraphPad, La Jolla, CA) software. In most cases, the Mann-Whitney (non-parametric) test was used to compare results. 


\section{Results}

\section{The Humoral Immune Response to MF59-Adjuvanted Vaccines Requires ASC but Not NLRP3 or Caspase-1}

NLRP3 inflammasome has been implicated in the adjuvant effect of alum. Therefore, we asked whether the NLRP3 inflammasome plays any role in mediating the adjuvant activity of MF59. We first determined the hemagglutination inhibition (HI) titers of WT, NLRP3 ${ }^{-/}$, $\mathrm{ASC}^{-/-}$, and caspase- $1^{-/-}$mice after vaccination with MF59-adjuvanted $\mathrm{H} 5 \mathrm{~N} 1$ subvirion vaccine. We found that $\mathrm{ASC}^{-/-}$mice, but not NLRP3 ${ }^{-/-}$ or caspase- $1^{-/}$mice, elicited significantly lower HI titers than WT mice (Fig. 4-1A). We next determined the serum levels of the different H5-specific antibody isotypes in immunized mice 3 weeks after a booster immunization. Consistent with the HI results, circulating levels of H5-specific IgM, IgG, IgG1, IgG2b, and IgG2c antibodies were comparably elevated in the serum of WT, NLRP3 $3^{--}$, and caspase- $1^{-/-}$mice (Fig. 4-1B-F). Immunized $\mathrm{ASC}^{-/-}$mice, however, elicited significantly less IgG, IgG1, IgG2b, and IgG2c antibodies than WT mice (Fig. 4-1C-F). Interestingly, circulating levels of $\mathrm{H} 5$-specific IgM antibodies in $\mathrm{ASC}^{-/-}$mice were comparable to those of WT mice (Fig. 4-1B).

As a control, we examined the $\mathrm{H} 5$-specific antibody response to the unadjuvanted vaccine in WT, NLRP3 ${ }^{-/-}, \mathrm{ASC}^{-/-}$and caspase- ${ }^{-/-}$mice (Fig. 4-2A $)$. $\mathrm{ASC}^{-/-}$mice robustly responded to the unadjuvanted vaccine indicating that the defective response observed with the MF59-adjuvanted vaccine was not due to a general defect in these mice to this particular inactivated influenza vaccine. In addition, infection with a sublethal dose of an attenuated $\mathrm{H} 5 \mathrm{~N} 1$ virus resulted in comparable ant-H5 IgG titers in WT and $\mathrm{ASC}^{-/}$mice as measured three weeks post infection (Fig. 4-2B). We next tested the antibody response to $\mathrm{H} 5 \mathrm{~N} 1$ vaccine adjuvanted with incomplete Freund's adjuvant (IFA) in WT and. ASC ${ }^{-/-}$ mice. Again, there were no significant differences in $\mathrm{H} 5$-specific IgG1 or IgG2c antibody titers (Fig. 4-2C-D). These results suggested that ASC is specifically required for the optimal generation of antigen-specific antibodies in response to MF59-adjuvanted H5 vaccines. In addition, class switching anti-H5 antibodies from IgM to IgG rather than antibody production itself was impaired in $\mathrm{ASC}^{-/-}$mice.

\section{ASC Is Required for Germinal Center B-Cell Formation after Immunization with MF59-Adjuvanted Vaccines}

Antibody class switching after vaccination or infection takes place mainly within the germinal center (GC) reaction in the draining lymph nodes (100). The observed decrease in $\mathrm{IgG}$ production in $\mathrm{ASC}^{-/-}$mice after MF59-adjuvanted $\mathrm{H} 5 \mathrm{~N} 1$ vaccination suggested a defect in the development of the $\mathrm{GC}$ reaction in these mice. To investigate this possibility, we immunized WT, NLRP3 ${ }^{-/-}, \mathrm{ASC}^{-/-}$, and caspase- $1^{-/-}$mice with the MF59-adjuvanted H5N1 vaccine and harvested the draining (inguinal) lymph nodes as well as sera 7 days later. 
A

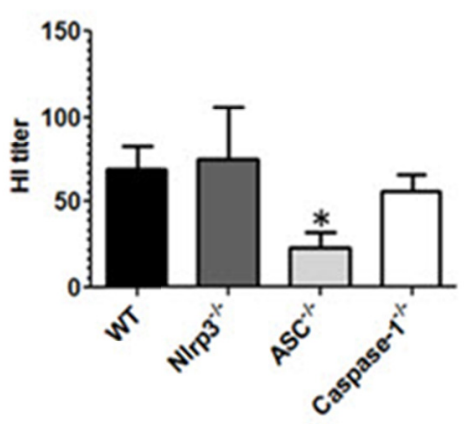

D

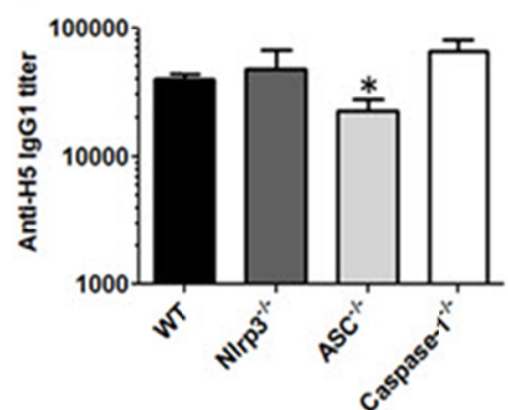

B

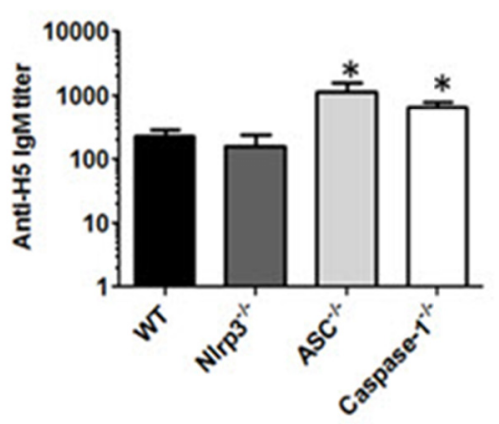

$\mathrm{E}$

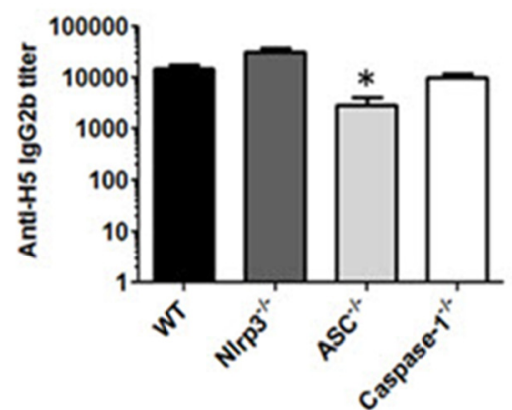

C

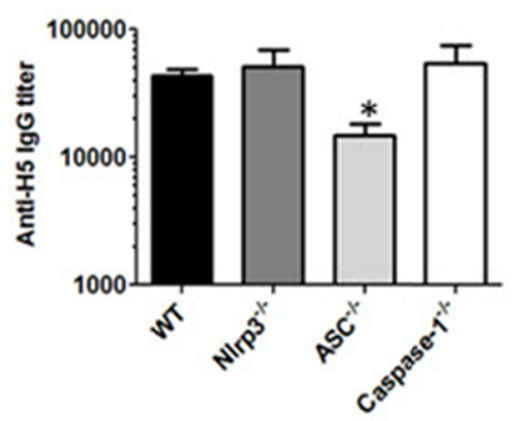

$\mathrm{F}$

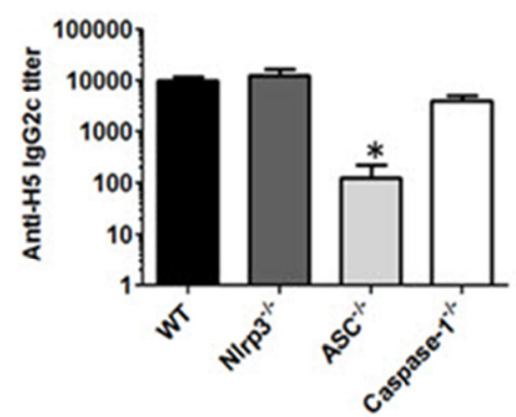

Figure 4-1. Defective humoral immune response to MF59-adjuvanted H5N1 vaccines in ASC knockout mice.

C57BL/6 wild-type (WT, $n=5), \mathrm{Nlrp}^{-/-}(\mathrm{n}=4), \mathrm{ASC}^{-/-}(\mathrm{n}=5)$, and caspase- $1^{-/-}(\mathrm{n}=5)$ mice immunized twice 3 weeks apart with 1 $\mu \mathrm{g}$ of H5N1 subvirion vaccine adjuvanted with MF59. Sera were collected 3 weeks after the boosting immunization. $(A)$ Mean serum hemagglutination inhibition (HI) titers. Mean circulating levels of H5-specific $(B) \operatorname{IgM},(C) \operatorname{IgG},(D) \operatorname{IgG1},(E) \operatorname{IgG} 2 \mathrm{~b}$, and $(F) \operatorname{IgG} 2 \mathrm{c}$ antibodies. The results are representative of at least 2 separate experiments. Statistically significant differences between WT and mutant groups are as indicated with asterisks; *, p $<0.05$. Error bars show standard error. 
A
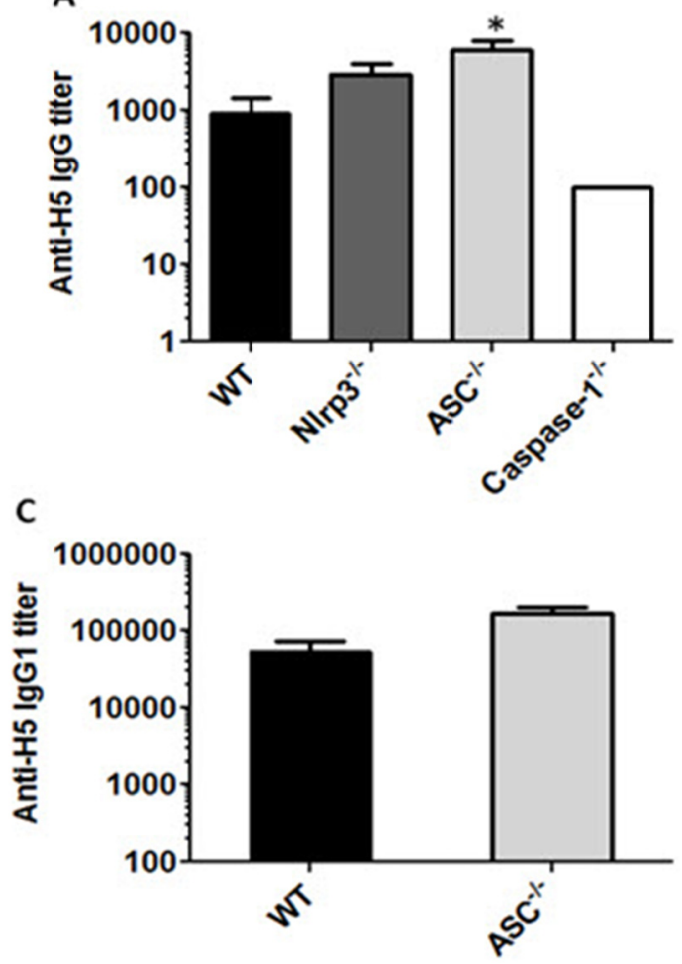

B

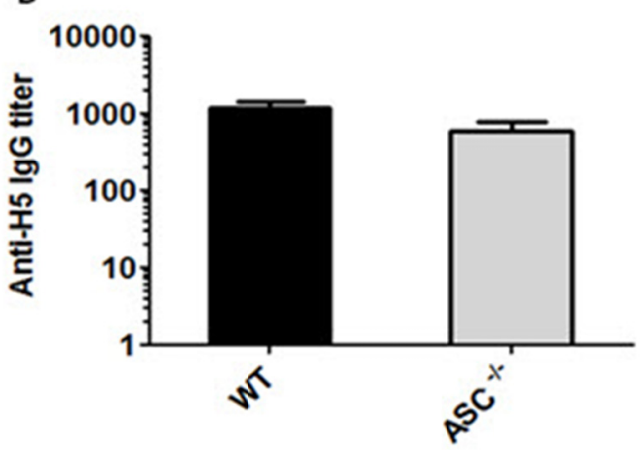

。

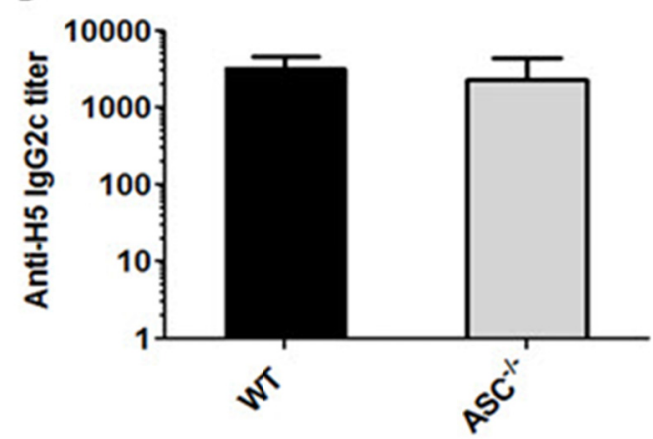

Figure 4-2. Anti-H5 antibody responses in $\mathrm{ASC}^{-/-}$are not generally defective.

Mean circulating levels of H5-specific IgG, IgG1 and IgG2c antibodies were determined by ELISA. $(A) \mathrm{C} 57 \mathrm{BL} / 6$ wild-type, $\mathrm{Nlrp} 3^{-/-}, \mathrm{ASC}^{-/-}$, and caspase- $1^{-/-}$mice immunized twice 3 weeks apart with $3 \mu \mathrm{g}$ of $\mathrm{H} 5 \mathrm{~N} 1$ subvirion vaccine adjuvanted in PBS. Sera were collected 3 weeks after the boosting immunization. (B) C57BL/6 wild-type and $\mathrm{ASC}^{-/-}$ mice were infected with 100 egg infectious dose 50 of the attenuated A/Vietnam/1203/2004 ( $\Delta$ HA) diluted in PBS to a final volume of $30 \mu 1$. Sera were collected 3 weeks after the infection. $(C-D) \mathrm{C} 57 \mathrm{BL} / 6$ wild-type and $\mathrm{ASC}^{-/-}$mice were primed with $1 \mu \mathrm{g}$ of $\mathrm{H} 5 \mathrm{~N} 1$ adjuvanted with IFA. Three weeks later, mice were boosted with $3 \mu \mathrm{g}$ of the H5N1 subvirion vaccine adjuvanted in PBS. Sera were collected 3 weeks after the boosting immunization. Error bars show standard error. 
H5-specific IgM and IgG antibodies were undetectable in the sera of $\mathrm{ASC}^{-/-}$mice, whereas both isotypes were detectable in the other three groups (Fig. 4-3A-B), indicating a delay in the induction of the antibody response in the $\mathrm{ASC}^{-/-}$mice. In agreement with this, WT, NLRP3 ${ }^{-/}$, and caspase- $1^{-/-}$mice all had significantly larger lymph nodes than $\mathrm{ASC}^{-/-}$mice, as evidenced by the difference in cellularity (Fig. 4-3D). We next looked at the frequency of GC B-cells in the draining ipsilateral lymph nodes. GC B-cells were defined by flow cytometry lymphocytes with the following expression profile; negative for surface CD4, CD8, and CD11b with low expression of surface IgD and high surface expression of B220 and FAS (CD195). We found that in WT, NLRP3 ${ }^{-/-}$, and caspase- $1^{-/-}$ mice, GC B-cells were comparably developed, accounting for $30-50 \%$ of the $\mathrm{B} 220^{+}$ B-cell population (Fig. 4-3C $\boldsymbol{E} \boldsymbol{E}$ ). On the other hand, GC B-cell frequencies in the $\mathrm{ASC}^{-/-}$ mice were significantly lower (Fig. 4-3D\&E).

The generation of a GC B-cell reaction requires the help of $\mathrm{CD} 4^{+} \mathrm{T}$-cells, so we asked whether they exhibited similar defects. Indeed, we found that absolute numbers of $\mathrm{CD}^{+} \mathrm{T}$-cells in the $\mathrm{ASC}^{-/-}$mice were significantly lower than in WT mice (Fig. 4-3F). These results show that ASC, independent of the caspase- 1 inflammasome, is required for the rapid induction of the antibody response to MF59-adjuvanted $\mathrm{H} 5 \mathrm{~N} 1$ vaccines. Moreover, the development of a GC reaction in the draining lymph nodes of $\mathrm{ASC}^{-/}$mice is impaired, explaining the defect observed in antibody isotype switching in these mice. This defect was not B-cells specific as CD4 T-cell frequencies were significantly lower in $\mathrm{ASC}^{-/-}$mice, potentially contributing to the germinal center phenotype.

\section{ASC Is Required for Robust Antigen-Specific Recall B-Cell Responses}

The ultimate outcome of a GC B-cell reaction is the generation of high-affinity, antigen-specific memory B-cells and long-lived plasma cells (PCs) (100). Upon antigen re-exposure, memory B-cells rapidly expand and differentiate into antibody-forming cells (AFCs). We therefore investigated whether the defect in GC B-cell development observed in $\mathrm{ASC}^{-/-}$mice after priming would, in turn, result in a defective $\mathrm{H} 5$-specific memory B-cell compartment. To test that, we primed WT, NLRP3 ${ }^{-/-}, \mathrm{ASC}^{-/}$, and caspase- $1^{-/-}$mice with one dose of MF59-adjuvanted H5N1 vaccine, and all mice were given a booster with the same vaccine in PBS 3 weeks later. Similar to our observations with the primary response, 5 days after antigen re-exposure, WT, NLRP3 ${ }^{-/-}$, and caspase- $1^{-/-}$mice had significantly larger draining lymph nodes than $\mathrm{ASC}^{-/-}$mice, and that was mirrored by a greater number of live cells (Fig. 4-4A). Also, GC B-cell numbers were significantly lower (Fig. 4-4B). Moreover, the number of CD138+ PCs in ASC $\mathrm{Al}^{-/}$ mice was significantly lower than in WT, NLRP3 $3^{-/-}$, and caspase- $1^{-/-}$mice (Fig. 4-4C). In agreement with the decreased number of CD138+ cells in $\mathrm{ASC}^{-/-}$mice after the boost, the number of H5-specific AFCs in the bone marrow was similarly lower (Fig. 4-4D). When we compared the IgG antibody titers in mice sera before and five days after the boost, $\mathrm{ASC}^{-/-}$mice showed a slight (2-fold) increase in antibody titer (Fig. 4-4E). In contrast, WT, NLRP3 ${ }^{-/}$, and caspase- $1^{-/-}$mice showed a significantly larger (5- to 6-fold) increase in $\mathrm{H} 5$-specific IgG titers after the boost, signifying a robust memory response in these mice (Fig. 4-4E). 
Figure 4-3. Defective germinal center B-cell development in $\mathrm{ASC}^{-/-}$after immunization with MF59-adjuvanted H5N1 subvirion vaccine.

C57BL/6 wild type, Nlrp3 $3^{-/-}, \mathrm{ASC}^{-/-}$and caspase- $1^{-/-}$mice immunized with $5 \mu \mathrm{g}$ of H5N1 subvirion vaccine adjuvanted with MF59. Serum and draining (inguinal) lymph nodes were collected 7 days after immunization. Mean circulating levels of H5-specific $(A) \operatorname{IgM}$ and $(B)$ IgG antibodies were determined as in Fig. 4-1. $(C)$, Representative FACS plots for lymphocytes from the draining lymph nodes stained with monoclonal antibodies to B220 and CD95 (FAS) on day 7 after immunization with $5 \mu \mathrm{g}$ of H5N1 subvirion vaccine adjuvanted with MF59. The gated population (circled in red) from each plot represents germinal center B-cells, which were defined as cells that were negative for surface CD4, CD8, and CD11b with low expression of surface IgD and high surface expression of B220 and FAS (CD195). (D), Mean number of live cells in the ipsilateral draining lymph node. $(E)$ Total number of germinal center B-cells as determined by FACS analysis of the cells with the phenotype defined in $(C) .(F)$, Total number of CD4 lymphocytes. Statistically significant differences between WT and mutant groups are as indicated with asterisks; ${ }^{*}, \mathrm{p}<0.05$. Error bars show standard error. 


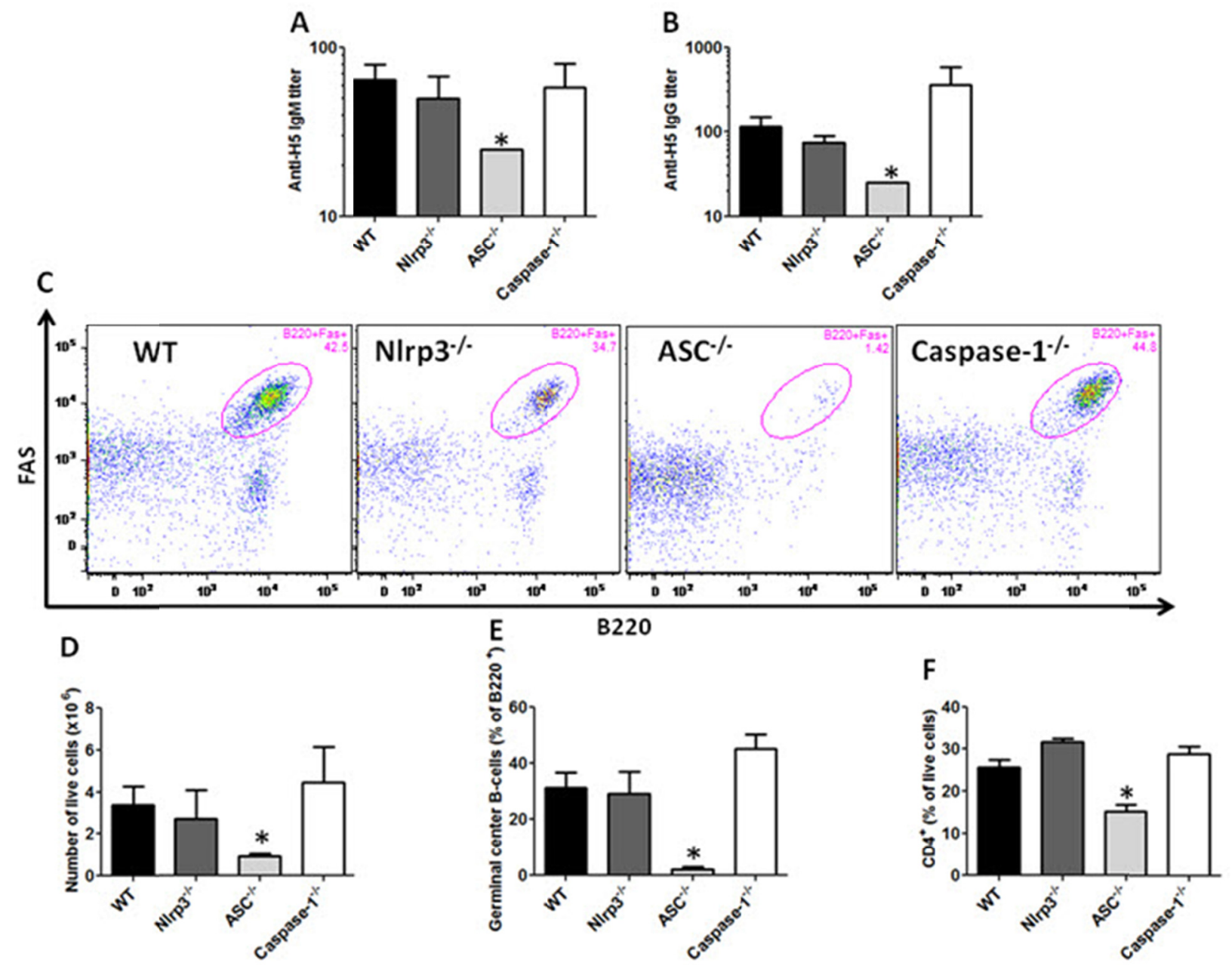



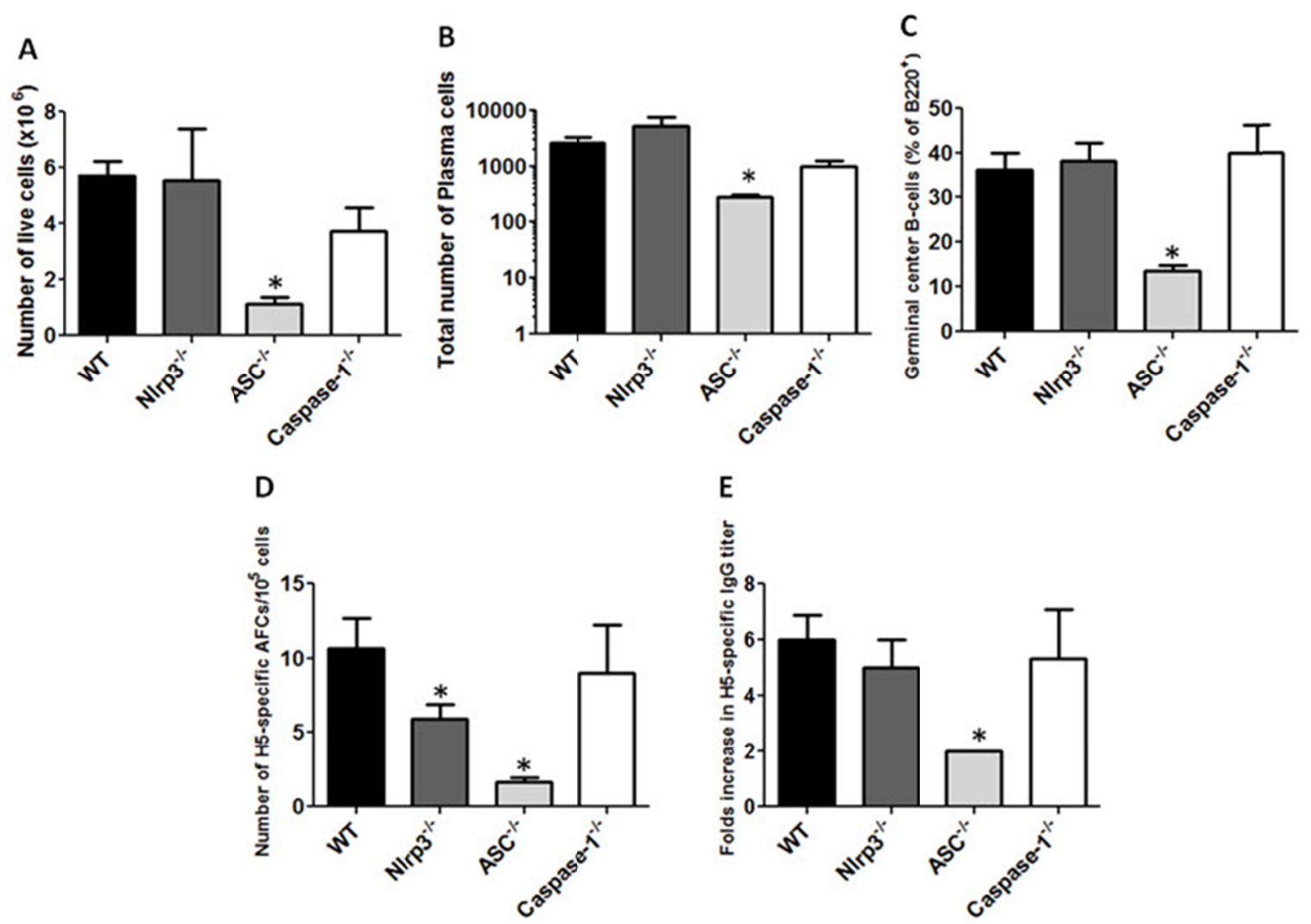

Figure 4-4. Poor $\mathrm{H} 5$-specific antibody recall responses in immunized $\mathrm{ASC}^{-/ 2}$ mice.

C57BL/6 wild-type, Nlrp3 ${ }^{-/}$, ASC ${ }^{-/-}$and caspase- $1^{-/-}$mice primed with $1 \mu \mathrm{g}$ of $\mathrm{H} 5 \mathrm{~N} 1$ subvirion vaccine adjuvanted with MF59 and boosted 3 weeks later with $5 \mu \mathrm{g}$ of H5N1 subvirion vaccine in PBS. Serum was collected prior to and 5 days after the boost. Ipsilateral draining lymph nodes and bone marrow were collected 5 days after the boost. $(A)$ Mean number of live cells in the draining ipsilateral draining lymph nodes. $(B)$ Mean number of plasma cells in the draining lymph nodes as determined by FACS analysis of cells that were negative for surface CD4, CD8, and CD11b with low expression of surface IgD and high surface expression of CD138. (C) Mean number of germinal center B-cells determined as in Fig. 4-3E. (D) Mean frequencies of H5-specific antibodyforming cells (AFCs) in the bone marrow of WT, $\mathrm{Nlrp3}^{-/-}, \mathrm{ASC}^{-/-}$, and caspase- $1^{-/-}$mice 5 days after antigen re-exposure. $(E)$ Folds increase in $\mathrm{H} 5$-specific IgG titers 5 days after antigen re-exposure. Statistically significant differences between WT and mutant groups are as indicated with asterisks; ${ }^{*}, \mathrm{p}<0.05$. Error bars show standard error. 
These results demonstrate that $\mathrm{ASC}$ is required for the optimal priming of antigen-specific B-cell memory responses with MF59-adjuvanted vaccines.

\section{ASC Expression in Dendritic Cells Is Required for Adequate Production of Inflammatory Cytokines}

We have shown that ASC-deficiency results in poor B and CD4 T cell responses to MF59-adjuvanted H5N1 subvirion vaccines. To further elucidate the mechanism by which ASC acts we started from the knowledge that the adjuvant effect of MF59 and alum at least partially depends on their ability to induce the secretion of chemokines responsible for recruiting inflammatory cells to the site of injection (103). It has been shown that within 48 hours of MF59 intramuscular injection, Dendritic cells or DCs internalize most of the adjuvant (72). We therefore investigated whether the defective antigen-specific antibody response in immunized $\mathrm{ASC}^{-/-}$mice was due to a defective inflammatory response by bone-marrow derived dendritic cells or BMDCs. To examine this, BMDCs from WT, NLRP3 ${ }^{-/}, \mathrm{ASC}^{-/-}$and caspase- $1^{-/-}$mice were incubated with MF59 and the secretion of proinflammatory cytokines in the culture supernatant was determined. For proinflammatory cytokines such as MIP1b and MIP2, WT BMDCs secreted significantly ( $\mathrm{p}=0.002$ and 0.005 , respectively) more as compared to BMDCs derived from $\mathrm{ASC}^{-/-}$but not significantly more than those derived from NLRP3 ${ }^{-/-}$and caspase- $1^{-/-}$mice (Fig. 4-5 A-B).

These results suggest that the inflammasome-independent role of ASC in generating the proper inflammatory environment after immunization may be the underlying cause of the defective induction of humoral immunity in the ASC-deficient mice.

\section{Discussion}

Unadjuvanted split and subunit $\mathrm{H} 5 \mathrm{~N} 1$ vaccines have been reproducibly shown to be poorly immunogenic in humans (78). The addition of alum adjuvant induced only marginal improvements (69). In contrast, oil-in-water based adjuvants (e.g., MF59) have been shown to prime for a robust and broadly cross-reactive antibody response to subvirion H5N1 vaccines with greatly reduced antigen requirements (69). MF59 has shown encouraging results in several influenza formulations and is licensed for use in seasonal influenza vaccines in Europe (74). However, the mechanism underlying the ability of MF59 and other adjuvants to stimulate the immune system remains poorly understood. In order to develop more defined next generation adjuvants, it is critical to determine the immunologic processes that are stimulated by MF59 and other efficacious adjuvants. Recent studies have shown that induction of antigen-specific antibody responses by commonly used vaccine adjuvants, such as alum and Freund's complete and incomplete adjuvants did not require signaling through Toll-like receptors (110).

However, the ability of alum and other particulate adjuvants to enhance IL-1 secretion via NLRP3 has been described (107). 
A

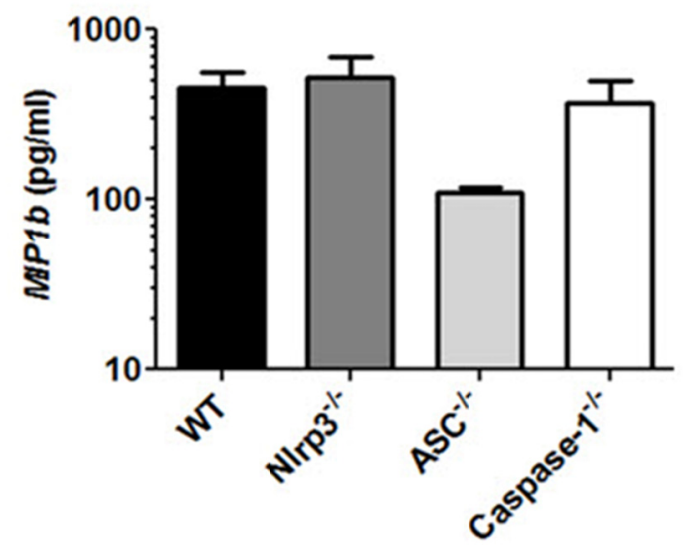

B

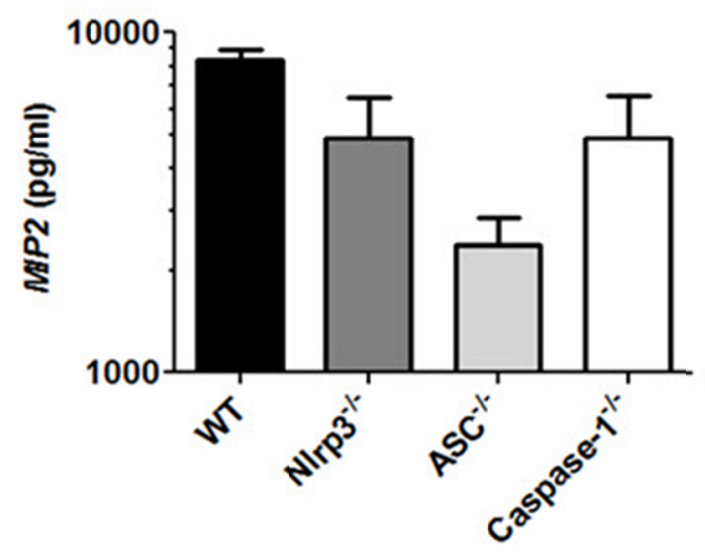

Figure 4-5. Defective inflammatory responses in $\mathrm{ASC}^{-/}$bone marrow-derived dendritic cells (BMDCs) to MF59.

BMDCs were stimulated for 24 hours with the MF59 in complete medium $(1: 100 \mathrm{~V} / \mathrm{V})$, and the quantities of MIP1 $\beta(A)$ and MIP2 $(B)$ were determined in cell culture supernatants. Tests were assayed in triplicate or quadruplicate. Error bars show standard error. 
In addition, some studies have shown that enhancement of antigen-specific humoral immunity by alum was also NLRP3-dependent (64), although later reports suggested that NLRP3 was not required $(65,66)$. Here, we found that the antibody response to H5N1 subunit influenza vaccines adjuvanted with MF59 critically depend on the presence of the adapter molecule ASC. After immunization with MF59-adjuvanted H5N1 subvirion vaccines, the amount of H5-specific antibodies of IgG1, IgG2b, and IgG2c subtypes were all significantly lower in $\mathrm{ASC}^{-/-}$mice than in WT mice. In contrast to studies with alum, we found that neither NLRP3 nor caspase-1 was required for the antigen-specific antibody responses to MF59-adjuvanted H5N1 influenza subvirion vaccines, indicating that the proposed role of ASC is independent of the caspase-1 inflammasome. It is interesting to note, however, that the murine antibody response to antigens emulsified in Freund's complete adjuvant has been shown to be unaffected by the absence of ASC (64). This suggests that even among emulsion-based adjuvants, the molecular requirement for the proper induction of an adaptive immune response varies. It is also possible that the complex nature of Freund's complete adjuvant affords immune stimulation through a number of redundant mechanisms.

Complexities of the response to adjuvants aside, our data support recent findings in the murine model of collagen-induced arthritis as well as in the development of experimental autoimmune encephalomyelitis that show an inflammasome-independent role for ASC in the induction of various forms of immunity and immunopathology (111, 112). There are several molecular mechanisms by which ASC may control the antigen-specific antibody response to MF59-adjuvanted vaccines. A key underlying feature in the $\mathrm{ASC}^{-/-}$mice was the significantly reduced production of key inflammatory cytokines by BMDCs as compared to WT derived cells after stimulation with MF59. Consistent with a defect in antigen presenting cells, it has been suggested that antigen-specific activation of T-cells by ASC-deficient BMDCs was significantly impaired (112). In line with this notion, after a single immunization with MF59-adjuvanted H5N1 vaccine, we observed a delayed antigen-specific antibody response and significantly lower numbers of $\mathrm{CD} 4^{+} \mathrm{T}$-cells in the draining lymph nodes vaccine in $\mathrm{ASC}^{-/}$but not NLRP3 ${ }^{-/-}$or caspase- $1^{-/-}$mice in comparison to their WT counterparts. In humans, the early development of an antigen specific $\mathrm{CD}^{+}{ }^{\mathrm{T}}$-cell response was shown to be a successful predictor of the robustness and persistence of an antibody response following MF59-adjuvanted H5N1 vaccination (102). We also demonstrated that the development of GC B-cells in the draining lymph nodes was significantly impaired in $\mathrm{ASC}^{-/-}$mice in comparison with that seen in WT, NLRP3 ${ }^{-/}$, and caspase $-1^{-/-}$mice after immunization. In addition, we showed that these defects resulted in a poor memory $\mathrm{B}$-cell response in $\mathrm{ASC}^{-/-}$mice. 


\section{CHAPTER 5. GENERAL CONCLUSIONS, LIMITATIONS AND FUTURE DIRECTIONS}

Prophylactic vaccination is based on a central feature of the adaptive immune system, immunological memory (113). Immunological memory represents the ability of the immune system to mount a robust recall response upon reexposure to a previously encountered antigen. The traditional microbial vaccines, live attenuated or whole inactivated pathogens, are strongly immunogenic due to their microbial content. However, the toxicity and risks associated with some of these preparations engendered the necessity to develop safer vaccines such as the purified protein preparations (114). The major challenge with these subunit protein vaccines is their poor immunogenicity. In order to efficiently design protein vaccines that stimulates protective immune responses, two factors must be considered; the choice of the antigen to be included in the vaccine preparation and the ability to stimulate the innate immune system. In the context of influenza vaccines, HA is clearly the antigen of choice because anti-HA antibodies can block the initiation of viral infection (45). As for the innate immune stimulation, the major advance achieved in this regard was the introduction of Adjuvants (60).

The word adjuvant is derived from the Latin word adjuvare, which means to aid (60). Adjuvants represent chemically and structurally diverse compounds. Traditionally, the use of adjuvants was primarily to enhance the quantitative immune response to vaccine antigens. For influenza vaccines, the benefits of adding adjuvants have been well documented (60). Basically, adjuvants increase the antibody titers in response to influenza vaccines in the general population and in individuals where the immune system is compromised. However, modulating the quality of the immune response has become an increasingly important aspect of the impact of adjuvants on vaccines in general. There are several factors through which adjuvants can possibly fine tune the immune responses to influenza vaccines; increasing the speed of the protective immune response, which is an essential measure in the event of an influenza pandemic; boosting the memory immune response, which provides a robust recall response upon reexposure; increasing the affinity of the HA-specific antibodies to increase the efficiency of virus neutralization; and increasing the breadth of the antibody response, which is important to generate cross reactive antibodies. A final quality that adjuvants can impart to the immune response to influenza vaccines is their ability to activate the most appropriate effector molecules. For example, among the different antibody isotypes, stimulating the secretion of $\operatorname{IgA}$ or $\operatorname{IgG} 2 \mathrm{a} / \mathrm{c}$ is arguably the most efficient in achieving protection (115).

Despite the fact that vaccines were introduced over two centuries ago, very few adjuvants have been licensed to be used with human vaccines (62). Over the past several decades many vaccine adjuvants, such as Freund's complete and incomplete adjuvants, have been tested and shown to be highly efficient in stimulating strong immune responses (62). The toxicity of these adjuvants, however, has limited their use with human vaccines (62). Currently, adjuvants that are licensed for use with human influenza vaccines were empirically developed. These adjuvants have been shown to be efficient and safe (60). The molecular details of the mechanisms mediating the adjuvanticity of clinically 
licensed adjuvants are still largely undefined (99). It is clear that dendritic cells (DCs) represent the most common target of many of these adjuvants. Effects of adjuvants on DCs vary from stimulating their migration, maturation, ability to present antigens to cytokine secretion. In order to be able to develop new or better adjuvants, the current adjuvants need to be mechanistically investigated. Over the past decade, many advances have been achieved in defining the molecular details of key pathways of the innate immune system. These discoveries have opened the door for scientists to explore the possible contribution of the innate immune system to the adjuvanticity of existing adjuvants.

Since their emergence in 1996, highly pathogenic H5N1 influenza viruses have evolved into at least 10 phylogenetically and antigenically different clades and multiple subclades (77). They pose a continuous threat of an influenza pandemic. Despite still being rare incidents, the mortality rate associated with human infections with these viruses is estimated to be $50-60 \%$ (77). This situation has been the catalyst for an increase in the amount of resources applied to preparedness activities for such a pandemic. Vaccine development has been the main focus of these activities. However, vaccine formulation has been a major challenge because these vaccines are not only required to effectively prime a population immunologically naïve to $\mathrm{H} 5$ antigens but also to protect against a broad spectrum of rapidly evolving viruses. This is because it is not known to which clade or subclade the potential pandemic H5N1 virus would belong to. The majority of the stockpiled pre-pandemic $\mathrm{H} 5 \mathrm{~N} 1$ vaccines are derived from clade 1 viruses (http://www.who.int/csr/disease/avian_influenza/guidelines/H5VaccineVirusUpdate2008 0214.pdf). Clinical trials indicated that compared to seasonal human influenza vaccines, $\mathrm{H} 5 \mathrm{~N} 1$ influenza vaccines elicit lower antibody titers at comparable antigen doses (78). Although currently there are no adjuvanted vaccines against seasonal influenza viruses licensed in the US, the poor immunogenicity of subvirion H5N1 influenza vaccines introduced the necessity of including adjuvants in the vaccine formulation.

\section{Adjuvants Enhance the Kinetics of Protective Antibody Responses to H5N1 Influenza Vaccines}

In Chapter 2, I investigated the impact of adjuvants on the speed of the immune response to pre-pandemic $\mathrm{H} 5 \mathrm{~N} 1$ influenza vaccines in the ferret model. The vaccine used was the subunit vaccine derived from A/Vietnam/1194/2004 (H5N1) and the adjuvants used in the study were MF59 and alum. Ferrets were immunized with vaccine in PBS or mixed with either of the two adjuvants at different time points prior to lethal challenge with A/Vietnam/1203/2004 (H5N1). We found that including an adjuvant in the vaccine formulation was essential for protection against lethality. This protection was, at least partially, attained when immunized ferrets were challenged as early as 7 days post immunization. This protection was lost when the pre-challenge incubation period was reduced to 4 days. Expectedly, extending this period to 14 or 21 days led to full protection against lethality and challenge associated morbidity. It is interesting to note that we were not able to detect $\mathrm{H} 5$ specific antibodies either by HI or ELISA in the sera of ferrets that survived after lethal challenge. 


\section{Implications and Limitations}

The general human population is immunologically naïve to H5N1 and other avian influenza viruses such as H7N7 and H9N2 as well. If an H5N1 avian influenza pandemic is to start soon, the first lines of defense would be antiviral drugs and stockpiled pre-pandemic vaccines. As for the antiviral drugs, assuming that the emerging virus retains the susceptibility for these drugs, the mass demand expected is very unlikely to be met by the existing stocks, which will be quickly depleted. The stockpiled pre-pandemic influenza vaccines would, therefore, play a crucial role in the response to any emerging epidemic and pandemic. Indeed, researchers, using mathematical modeling, have predicted that pre-pandemic vaccines when utilized at the early stages of the pandemic would significantly limit the virus spread $(84,116)$. The protection would even be greater when other measures such as social distancing and travel restriction are applied. These theoretical studies are assuming that it would take at least 2 immunizations and 14 days post-immunization to attain full protection from lethality. Our data show that the protection can be attained in a shorter period (7 days) after a single immunization, which in turn means that a more efficient control of any emerging influenza pandemic can be achieved using adjuvanted pre-pandemic vaccines.

The poor immunogenicity displayed by the unadjuvanted pre-pandemic H5N1 vaccines puts into question the suboptimal utilization of adjuvants in the context of such vaccines. The two adjuvants used in the study are both licensed to be used in human influenza vaccines. Several studies have indicated that alum is inferior to water-in-oil emulsion based emulsions such as MF59 in inducing strong antibody response to influenza vaccines. These conclusions were derived from clinical studies or pre-clinical studies performed in mice. In our studies, however, alum has shown an equivalent, if not superior, ability to induce a protective antibody response to the H5N1 subunit influenza vaccine. The difference in the animal model used may be the reason for what we observed.

The challenge virus we used in our study is antigenically indistinguishable from the vaccine parent strain i.e. homologous challenge. This is most likely will not be the case and therefore the protection observed will largely depend on how antigenically distant the vaccine parent strain from challenge virus strains.

\section{Future Directions}

While showing that a single immunization with an adjuvanted pre-pandemic $\mathrm{H} 5 \mathrm{~N} 1$ vaccine can provide protection against lethality in a relatively short period (7 days) is important, it will be equally essential to determine how long such protection would last. Also, it will be important to determine whether this protection will block virus transmission.

Germann and colleagues have predicted that even the use of a poorly matched pre-pandemic vaccine would be helpful in the containment plans in the event of an 
influenza pandemic (84). Their rational was that this poorly matched vaccine could slow virus spread as much as possible until a well matched vaccine based on the emergent human pandemic virus could be deployed. Therefore, it will be important to determine the extent of protection, if any, that can be achieved in the heterologous challenge scenario.

\section{Adjuvants' Impact on the Breadth of the Antibody Responses to Subunit H5N1 Influenza Vaccines}

In Chapter 3, I studied the impact of the adjuvants MF59 and alum on the breadth of the antibody responses to subunit $\mathrm{H} 5 \mathrm{~N} 1$ vaccines. The results showed that MF59-adjuvanted H5N1 vaccines elicits a quantitatively greater $\mathrm{H} 5$-specific antibody response than the alum-adjuvanted or the unadjuvanted vaccine. These titers were consistent with the ability of MF59-adjuvanted vaccine to prime for more robust H5-specific B-cell responses. However, the specificity of these antibodies was not different. Using $\mathrm{H} 5$ antigen microarray, the binding pattern of purified $\operatorname{IgG}$ immunoglobulins from mice immunized with MF59- or alum-adjuvanted or the unadjuvanted vaccine was similar. Additionally, competitive inhibition studies using antibodies that target prominent epitopes on the HA globular head indicated that these epitopes were targeted to a similar extent.

\section{Implications and Limitations}

The primary challenging issue with influenza vaccines in general is that their efficacies drop as the circulating virus evolves away from the vaccine parent strain itself. Therefore, the H5N1 vaccines stockpiled in anticipation of a pandemic may lose efficacy and usefulness over time due to antigenic drift of circulating strains. Consequentially, it was suggested to use the stockpiled $\mathrm{H} 5 \mathrm{~N} 1$ vaccines to prime the population in this inter-pandemic period (http://www.who.int/immunization/sage/SAGE_H5N1_26Mayb.pdf). This priming can provide some protection against a potentially emerging pandemic. As we discussed above, there is a high probability of including adjuvants such as the oil-in-water emulsion MF59 in this pre-pandemic H5N1 vaccine priming. Therefore, it is essential to study the impact of adjuvants on $3 \mathrm{key}$ criteria in order to be able to assess the potential usefulness of this priming:

1) The generation of B-cell memory.

2) The kinetics and magnitude of the recall response after the booster immunization.

3) The breadth of the antibody response.

As for the first two points, studies by Galli and colleagues have shown that priming with MF59 adjuvanted-H5N1 vaccines primed for a greater B-cell memory responses compared to plain vaccine primed or unprimed individuals (117). However, it is important to note that in this study the researchers estimated the memory responses by 
examining the differences in recall responses after a booster immunization. Interestingly, the booster immunization vaccine was derived from clade 1 (A/Vietnam/1194/2004, $\mathrm{H} 5 \mathrm{~N} 1)$ virus while the priming immunization vaccine was derived from a clade 0 (A/duck/Singapore/1997, H5N3) virus i.e. heterologous prime-boost. In a similar study, Leroux-Roels and colleagues showed that another oil-in-water emulsion adjuvant, AS03, enhanced the initial priming effect of pre-pandemic H5N1 influenza vaccination enabling a rapid humoral response to single dose boosting with a heterologous strain (118). Both of the above mentioned clinical studies and others showed that priming with an oil-inwater emulsion adjuvanted $\mathrm{H} 5$ antigen can result in a vastly improved response to subsequent vaccination, even if this subsequent vaccination is with a drifted strain (119). In contrast, at least in one of these studies, priming without adjuvant seemed to inhibit the response to subsequent vaccination with the heterologous strain (118). Moreover, including MF59, in multiple clinical trials with both seasonal and pandemic vaccines, enhanced antibody titers not only in response to homologous influenza strains but also to antigenically distinct virus strains $(93,94)$. Taken together, these results suggest that including the oil-in-water emulsion adjuvants with the pre-pandemic $\mathrm{H} 5 \mathrm{~N} 1$ vaccines increases the breadth of the responding B-cells. In line with this notion, Khurana et al. screened the binding of sera from participants in two separate clinical trials to linear epitopes spanning the length of HA and NA and observed that including MF59 adjuvant with $\mathrm{H} 5 \mathrm{~N} 1$ vaccines expanded the repertoire of responding antibodies (59). Our data contrast the proposed adjuvant-mediated "epitope spreading" of the responding antibodies by MF59-adjuvanted H5N1 vaccines. There are 2 key points that might explain this apparent discrepancy:

1) The assay used to estimate the breadth of the antibody response. The vast majority of clinical studies utilize $\mathrm{HI}$ as a standard assay to evaluate vaccine mediated antibody responses in the sera of participants. The sensitivity of the assay may preclude the detection of the small proportion of antibodies reacting with the antigenically distinct variants in the generally weak responses elicited by unadjuvanted vaccines.

2) Differences in the binding affinity of antibodies from the adjuvanted vs. the unadjuvanted vaccine responses. Our results showed that responding B-cells to the MF59-adjuvanted H5N1 vaccines experienced a greater degree of affinity maturation compared to B-cells activated by the unadjuvanted vaccine. While we did not directly assess the differences in binding affinities between these polyclonal responses, these differences, if existed, might contribute to the "epitope spreading" observed sera derived from individuals that received the MF59-adjuvanted vaccine.

\section{Future Directions}

One of the questions that remain unanswered is how adjuvants impact the diversity of the clonal B-cell responses. In the course of comparing the extent of affinity maturation of between adjuvanted and unadjuvanted B-cell responses, we sequenced the 
immunoreceptor genes of activated B-cells. We believe that careful examination of these sequences would provide a preliminary insight regarding the clonal diversity within adjuvanted vs. unadjuvanted B-cell responses. Tiller and colleagues in a method report described the cloning and expression of murine Ig genes from single B cells (97). Performing a similar analysis on adjuvanted vs. unadjuvanted B-cell responses would not only allow for examining the clonal diversity of these responses but also their functional diversity as well. The latter would be defined by the differences in specificity and affinity of expressed immunoglobulins.

\section{Inflammasome-Independent Role of the Apoptosis-Associated Speck-Like Protein Containing CARD (ASC) in the Adjuvant Effect of MF59}

In Chapter 4, I investigated the possible roles of a key innate immune pathway, the NLRP3 inflammasome, in the adjuvanticity of the oil-in-water emulsion based adjuvant MF59 (120). We showed that, unlike the case for alum, MF59 does not require NLRP3 to exert its adjuvant effect. We showed that the H5-specific antibody response to MF59-adjuvanted H5N1 vaccine was not compromised in mice genetically deficient in NLRP3 and also in mice deficient in caspases-1, a key molecule in the NLRP3 inflammasome. It was surprising, however, to see that the adapter molecule that bridges the interaction between NLRP3 and caspases-1, ASC, is indispensable for optimal MF59-mediated anti-H5 antibody response. Mice genetically deficient in ASC elicited significantly less anti-H5 HI and IgG titers compared to their wild type counterparts. These defects were not observed in the antibody response to the unadjuvanted vaccine or following sublethal $\mathrm{H} 5 \mathrm{~N} 1$ virus infection. Also the responses were intact when IFA and not MF59 was the adjuvant. We next showed that the decreased antibody titers are due to the failure of $\mathrm{ASC}^{-/-}$mice to develop a proper germinal center reaction in the draining lymph nodes following immunization. Finally, we showed that the production of the pro-inflammatory chemokines such as MIP-1b and MIP2 were decreased in BMDCs derived from $\mathrm{ASC}^{-/-}$compared to wild type cells upon treatment with MF59.

\section{Implications and Limitations}

Similar to some commonly used vaccine adjuvants, the oil-in-water emulsion adjuvant MF59 functions via its ability to limit antigen dispersion from the injection site and consequentially promoting its uptake by professional APCs (60). This depot effect might be mediated by the oil component of the adjuvant. Another important aspect of the adjuvanticity of MF59 is the ability to trigger local inflammatory response that leads to the recruitment of various inflammatory cells to the injection site (104). The molecular mechanism underlying this immune stimulation is yet to be fully elucidated. In CFA, for example, the mineral oil component mediates the depot effect while the mycobacterial cell wall components would provide the ligands for the innate immune receptors.

The implication of the NLRP3 inflammasome in the immunostimulatory effect of alum has fueled the speculation of the role that this novel innate immune pathway might 
be playing with other vaccine adjuvants (121). Indeed, in vitro studies revealed that particulate adjuvants such as the poly (lactic-co-glycolic acid) activated the NLRP3 inflammasome as well (122). However, studies with mice that are genetically deficient in the cytosolic sensor NLRP3 showed minimal effect on the antibody responses stimulated by these adjuvants $(65,123)$. Our studies with MF59 clearly show that the NLRP3 inflammasome is dispensable for the MF59-mediated immune stimulation. However, our data indicate that there is an inflammasome-independent pathway in which the adapter protein ASC is an essential component plays a vital role in the adjuvant effect of MF59. The promising nature of MF59 use in humans and the identification of ASC as a critical factor for the proper induction of the adaptive immune response to MF59-adjuvanted vaccines would have an important implications for the formulation of future influenza vaccines.

\section{Future Directions}

Our knowledge of the innate immune system has expanded over the last decade (124). Currently there are no innate immune receptors directly implicated in the adjuvant effect of MF59 and other oil-in-water emulsion-based adjuvants (99). While our finding of the involvement of the adapter molecule ASC in the MF59 mechanism of action represents an important advancement in this regard, it will be essential to identify the upstream molecule or molecules that are also involved. Identifying these receptors will allow for the rational design of new vaccine adjuvants.

While our data indicate that ASC plays a role in the generation of the inflammatory response by innate immune cells in response to MF59 treatment. It will be important to determine the possible role of ASC within other immune cells such as B-cells and T-cells. Apart from that, the possible contribution of another major innate immune recognition system, the toll-like receptors (TLRs), in the adjuvant effect of MF59 has not been examined so far. 


\section{LIST OF REFERENCES}

1. T. K. Cheung, L. L. Poon, Ann N Y Acad Sci 1102, 1 (Apr, 2007).

2. R. G. Webster, W. J. Bean, O. T. Gorman, T. M. Chambers, Y. Kawaoka, Microbiol Rev 56, 152 (Mar, 1992).

3. J. J. Skehel, D. C. Wiley, Annu Rev Biochem 69, 531 (2000).

4. S. J. Gamblin, J. J. Skehel, J Biol Chem 285, 28403 (Sep 10, 2010).

5. R. A. Fouchier et al., J Virol 79, 2814 (Mar, 2005).

6. L. H. Pinto, L. J. Holsinger, R. A. Lamb, Cell 69, 517 (May 1, 1992).

7. A. Fernandez-Sesma, Infect Disord Drug Targets 7, 336 (Dec, 2007).

8. K. Subbarao, T. Joseph, Nat Rev Immunol 7, 267 (Apr, 2007).

9. W. Chen et al., Nat Med 7, 1306 (Dec, 2001).

10. A. Mikulasova, E. Vareckova, E. Fodor, Acta Virol 44, 273 (Oct, 2000).

11. A. E. Fiore et al., MMWR Recomm Rep 57, 1 (Aug 8, 2008).

12. A. J. Hay, V. Gregory, A. R. Douglas, Y. P. Lin, Philos Trans R Soc Lond B Biol Sci 356, 1861 (Dec 29, 2001).

13. R. J. Webby, R. G. Webster, Science 302, 1519 (Nov 28, 2003).

14. G. Neumann, T. Noda, Y. Kawaoka, Nature 459, 931 (Jun 18, 2009).

15. T. M. Tumpey, J. A. Belser, Annu Rev Microbiol 63, 79 (2009).

16. E. D. Kilbourne, J Infect Dis 176 Suppl 1, S29 (Aug, 1997).

17. MMWR Morb Mortal Wkly Rep 58, 400 (Apr 24, 2009).

18. F. S. Dawood et al., N Engl J Med 360, 2605 (Jun 18, 2009).

19. G. J. Smith et al., Nature 459, 1122 (Jun 25, 2009).

20. G. Neumann, M. A. Green, C. A. Macken, J Gen Virol 91, 1984 (Aug, 2010).

21. A. N. Abdel-Ghafar et al., N Engl J Med 358, 261 (Jan 17, 2008). 
22. T. R. Maines et al., Immunol Rev 225, 68 (Oct, 2008).

23. J. S. Peiris, M. D. de Jong, Y. Guan, Clin Microbiol Rev 20, 243 (Apr, 2007).

24. D. Vijaykrishna et al., PLoS Pathog 4, e1000161 (2008).

25. C. Li et al., Proc Natl Acad Sci US A 107, 4687 (Mar 9, 2010).

26. R. Bodewes, G. F. Rimmelzwaan, A. D. Osterhaus, Expert Rev Vaccines 9, 59 (Jan, 2010).

27. J. S. Peiris, K. P. Hui, H. L. Yen, Curr Opin Immunol 22, 475 (Aug, 2010).

28. I. A. Wilson, J. J. Skehel, D. C. Wiley, Nature 289, 366 (Jan 29, 1981).

29. J. Stevens et al., Science 312, 404 (Apr 21, 2006).

30. T. Ichinohe, A. Iwasaki, H. Hasegawa, Expert Rev Vaccines 7, 1435 (Nov, 2008).

31. D. C. Hargreaves, R. Medzhitov, J Clin Immunol 25, 503 (Nov, 2005).

32. T. D. Kanneganti, Nat Rev Immunol 10, 688 (Oct, 2010).

33. S. Manicassamy, B. Pulendran, Semin Immunol 21, 185 (Aug, 2009).

34. A. K. Heer et al., J Immunol 178, 2182 (Feb 15, 2007).

35. J. M. Lund et al., Proc Natl Acad Sci U S A 101, 5598 (Apr 13, 2004).

36. K. Schroder, J. Tschopp, Cell 140, 821 (Mar 19, 2010).

37. P. G. Thomas et al., Immunity 30, 566 (Apr 17, 2009).

38. W. Gerhard, Curr Top Microbiol Immunol 260, 171 (2001).

39. A. H. Ellebedy et al., Vaccine 12;29(17):3335-9 (Sep 15, 2010).

40. N. L. Varich et al., J Gen Virol 90, 1730 (Jul, 2009).

41. R. E. Dutch, T. S. Jardetzky, R. A. Lamb, Biosci Rep 20, 597 (Dec, 2000).

42. C. J. Wei et al., Science 329, 1060 (Aug 27, 2010).

43. M. R. Sandbulte et al., PLoS Med 4, e59 (Feb, 2007).

44. J. Fan et al., Vaccine 22, 2993 (Aug 13, 2004). 
45. S. A. Valkenburg et al., Microbes Infect, 13(5):489-501 (Feb 1, 2011).

46. J. Stambas et al., Pharmacol Ther 120, 186 (Nov, 2008).

47. M. Prabakaran et al., PLoS One 4, e4566 (2009).

48. A. H. Ellebedy, R. J. Webby, Vaccine 27 Suppl 4, D65 (Nov 5, 2009).

49. T. Jefferson et al., Cochrane Database Syst Rev, CD001269 (2010).

50. T. Jefferson et al., Cochrane Database Syst Rev, CD004876 (2010).

51. S. R. Mostow, S. C. Schoenbaum, W. R. Dowdle, M. T. Coleman, H. S. Kaye, Bull World Health Organ 41, 525 (1969).

52. F. B. Peck, Jr., JAMA 206, 2277 (Dec 2, 1968).

53. J. L. Bresson et al., Lancet 367, 1657 (May 20, 2006).

54. M. D. Wareing, G. A. Tannock, Vaccine 19, 3320 (May 14, 2001).

55. A. S. Monto et al., N Engl J Med 361, 1260 (Sep 24, 2009).

56. G. J. Nabel, A. S. Fauci, Nat Med 16, 1389 (Dec, 2010).

57. V. Aimanianda, J. Haensler, S. Lacroix-Desmazes, S. V. Kaveri, J. Bayry, Trends Pharmacol Sci 30, 287 (Jun, 2009).

58. P. R. Dormitzer et al., Immunol Rev 239, 167 (Jan, 2011).

59. S. Khurana et al., Sci Transl Med 2, 15 ra5 (Jan 20, 2010).

60. R. L. Atmar, W. A. Keitel, Curr Top Microbiol Immunol 333, 323 (2009).

61. A. S. McKee, M. K. MacLeod, J. W. Kappler, P. Marrack, BMC Biol 8, 37 (2010).

62. F. R. Vogel, Clin Infect Dis 30 Suppl 3, S266 (Jun, 2000).

63. S. L. Hem, H. Hogenesch, Expert Rev Vaccines 6, 685 (Oct, 2007).

64. S. C. Eisenbarth, O. R. Colegio, W. O'Connor, F. S. Sutterwala, R. A. Flavell, Nature 453, 1122 (Jun 19, 2008).

65. R. Spreafico, P. Ricciardi-Castagnoli, A. Mortellaro, Eur J Immunol 40, 638 (Mar, 2010). 
66. A. S. McKee et al., J Immunol 183, 4403 (Oct 1, 2009).

67. L. Franchi, G. Nunez, Eur J Immunol 38, 2085 (Aug, 2008).

68. W. A. Keitel et al., J Infect Dis 198, 1309 (Nov 1, 2008).

69. D. I. Bernstein et al., J Infect Dis 197, 667 (Mar 1, 2008).

70. H. J. Ehrlich et al., N Engl J Med 358, 2573 (Jun 12, 2008).

71. M. Dupuis, D. M. McDonald, G. Ott, Vaccine 18, 434 (Oct 14, 1999).

72. M. Dupuis et al., Cell Immunol 186, 18 (May 25, 1998).

73. F. Mosca et al., Proc Natl Acad Sci U S A 105, 10501 (Jul 29, 2008).

74. D. T. O'Hagan, Expert Rev Vaccines 6, 699 (Oct, 2007).

75. K. G. Nicholson et al., Lancet 357, 1937 (Jun 16, 2001).

76. R. L. Atmar et al., Clin Infect Dis 43, 1135 (Nov 1, 2006).

77. S. Sambhara, G. A. Poland, Annu Rev Med 61, 187 (2010).

78. J. J. Treanor, J. D. Campbell, K. M. Zangwill, T. Rowe, M. Wolff, N Engl J Med 354, 1343 (Mar 30, 2006).

79. J. Lin et al., Lancet 368, 991 (Sep 16, 2006).

80. D. Middleton et al., J Virol 83, 7770 (Aug, 2009).

81. P. D. Reuman, S. Keely, G. M. Schiff, J Virol Methods 24, 27 (Apr-May, 1989).

82. I. M. Longini, Jr. et al., Science 309, 1083 (Aug 12, 2005).

83. N. M. Ferguson et al., Nature 437, 209 (Sep 8, 2005).

84. T. C. Germann, K. Kadau, I. M. Longini, Jr., C. A. Macken, Proc Natl Acad Sci U $S A$ 103, 5935 (Apr 11, 2006).

85. L. Y. Lee et al., J Clin Invest 118, 3478 (Oct, 2008).

86. A. H. Ellebedy et al., Clin Vaccine Immunol 17, 1998 (Dec, 2010).

87. Emerg Infect Dis 11, 1515 (Oct, 2005). 
88. I. Stephenson et al., J Infect Dis 191, 1210 (Apr 15, 2005).

89. G. Del Giudice et al., Vaccine 24, 3063 (Apr 12, 2006).

90. G. Milne, J. Kelso, H. Kelly, J R Soc Interface 7, 573 (Apr 6, 2010).

91. I. Leroux-Roels et al., Lancet 370, 580 (Aug 18, 2007).

92. H. L. Yen, R. G. Webster, Curr Top Microbiol Immunol 333, 3 (2009).

93. A. Banzhoff et al., Influenza Other Respi Viruses 2, 243 (Nov, 2008).

94. F. Ansaldi et al., Vaccine 26, 1525 (Mar 17, 2008).

95. B. Baras et al., PLoS One 3, e1401 (2008).

96. L. Malherbe, C. Haus1, L. Teyton, M. G. McHeyzer-Williams, Immunity 21, 669 (Nov, 2004).

97. T. Tiller, C. E. Busse, H. Wardemann, J Immunol Methods 350, 183 (Oct 31, 2009).

98. A. Wack et al., Vaccine 26, 552 (Jan 24, 2008).

99. R. L. Coffman, A. Sher, R. A. Seder, Immunity 33, 492 (Oct 29, 2010).

100. L. J. McHeyzer-Williams, M. G. McHeyzer-Williams, Annu Rev Immunol 23, 487 (2005).

101. R. W. Maul, P. J. Gearhart, Immunol Res 47, 113 (Jul, 2010).

102. G. Galli et al., Proc Natl Acad Sci US A 106, 3877 (Mar 10, 2009).

103. S. Calabro et al., Vaccine 29, 1812 (Feb 17, 2011).

104. A. Seubert, E. Monaci, M. Pizza, D. T. O'Hagan, A. Wack, J Immunol 180, 5402 (Apr 15, 2008).

105. T. D. Kanneganti, M. Lamkanfi, G. Nunez, Immunity 27, 549 (Oct, 2007).

106. H. Li, S. B. Willingham, J. P. Ting, F. Re, J Immunol 181, 17 (Jul 1, 2008).

107. H. Li, S. Nookala, F. Re, J Immunol 178, 5271 (Apr 15, 2007).

108. M. Lamkanfi, T. D. Kanneganti, Int J Biochem Cell Biol 42, 792 (Jun, 2010). 
109. M. Lamkanfi, R. K. Malireddi, T. D. Kanneganti, J Biol Chem 284, 20574 (Jul 31, 2009).

110. A. L. Gavin et al., Science 314, 1936 (Dec 22, 2006).

111. P. J. Shaw et al., J Immunol 184, 4610 (May 1, 2010).

112. S. K. Ippagunta et al., J Biol Chem 285, 12454 (Apr 16, 2010).

113. F. Sallusto, A. Lanzavecchia, K. Araki, R. Ahmed, Immunity 33, 451 (Oct 29, 2010).

114. W. E. Beyer, A. M. Palache, A. D. Osterhaus, Clin Drug Investig 15, 1 (1998).

115. A. S. McKee, M. W. Munks, P. Marrack, Immunity 27, 687 (Nov, 2007).

116. N. M. Ferguson et al., Nature 442, 448 (Jul 27, 2006).

117. G. Galli et al., Proc Natl Acad Sci US A 106, 7962 (May 12, 2009).

118. I. Leroux-Roels et al., Vaccine 28, 849 (Jan 8, 2010).

119. I. Stephenson et al., N Engl J Med 359, 1631 (Oct 9, 2008).

120. A. H. Ellebedy et al., Proc Natl Acad Sci US A 108, 2927 (Feb 15, 2011).

121. E. De Gregorio, U. D'Oro, A. Wack, Curr Opin Immunol 21, 339 (Jun, 2009).

122. F. A. Sharp et al., Proc Natl Acad Sci US A 106, 870 (Jan 20, 2009).

123. J. Harris, F. A. Sharp, E. C. Lavelle, Eur J Immunol 40, 634 (Mar, 2010).

124. N. W. Palm, R. Medzhitov, Immunol Rev 227, 221 (Jan, 2009). 


\section{APPENDIX A. IMPACT OF PRIOR SEASONAL INFLUENZA VACCINATION AND INFECTION ON PANDEMIC A (H1N1) INFLUENZA VIRUS REPLICATION IN FERRETS}
A. H. Ellebedy et al., Vaccine 12;29(17):3335-9 (Sep 15, 2010).
Follow this link:
http://www.sciencedirect.com/science? ob=MImg\& imagekey=B6TD4-511GFHF-1-
$1 \&$ cdi $=5188 \&$ user $=930789 \&$ pii $=$ S0264410X $10012363 \&$ origin $=$ gateway $\&$ coverDa te $=04 \% 2 F 12 \% 2 F 2011 \&$ sk $=999709982 \& v i e w=c \& w c h p=d G L z V z z-$
zSkzV\&md5=06b47b0ebc8f4bada165874d4c77fc12\&ie $=/$ sdarticle.pdf 


\section{APPENDIX B. CONTEMPORARY SEASONAL INFLUENZA A (H1N1) VIRUS INFECTION PRIMES FOR A MORE ROBUST RESPONSE TO SPLIT INACTIVATED PANDEMIC INFLUENZA A (H1N1) VIRUS VACCINATION IN FERRETS}

A. H. Ellebedy et al., Clin Vaccine Immunol 17, 1998 (Dec, 2010).

Follow this link:

http://cvi.asm.org/cgi/reprint/17/12/1998 


\section{VITA}

Ali H. Ellebedy was born in Assiout, Egypt in 1982. He graduated from Ahmed Lotfy El-sayed High School in Cairo, Egypt in May 1999 and from the College of Pharmacy at Cairo University in the spring of 2004. Following graduation, he worked as a teaching and research assistant at the Department of Microbiology and Immunology, College of Pharmacy, Cairo University. He began his studies in the Interdisciplinary Program of Biomedical Sciences at the University of Tennessee Health Science Center in August 2006 and performed his dissertation research under the supervision of Dr. Richard Webby at St. Jude Children's Research Hospital. He received his PhD with a concentration in Microbial Pathogenesis, Immunology and Inflammation in May 2011. 